\title{
NLO electroweak automation and precise predictions for $W+$ multijet production at the LHC
}

\author{
S. Kallweit, ${ }^{a, c}$ J.M. Lindert, ${ }^{a}$ P. Maierhöfer, ${ }^{a, b}$ S. Pozzorini ${ }^{a}$ and M. Schönherr ${ }^{a, b}$ \\ ${ }^{a}$ Physik-Institut, Universität Zürich, \\ Winterthurerstrasse 190, CH-8057 Zürich, Switzerland \\ ${ }^{b}$ Institute for Particle Physics Phenomenology, Durham University, \\ Durham DH1 3LE, U.K. \\ ${ }^{c}$ Institut für Physik \& PRISMA Cluster of Excellence, Johannes Gutenberg Universität, \\ 55099 Mainz, Germany \\ E-mail: kallweit@physik.uzh.ch, lindert@physik.uzh.ch, \\ philipp.maierhoefer@durham.ac.uk, pozzorin@physik.uzh.ch, \\ marek.schoenherr@physik.uzh.ch
}

ABSTRACT: We present a fully automated implementation of next-to-leading order electroweak (NLO EW) corrections in the OPENLOOPS matrix-element generator combined with the Sherpa and Munich Monte Carlo frameworks. The process-independent character of the implemented algorithms opens the door to NLO QCD+EW simulations for a vast range of Standard Model processes, up to high particle multiplicity, at current and future colliders. As a first application, we present NLO QCD+EW predictions for the production of positively charged on-shell $W$ bosons in association with up to three jets at the Large Hadron Collider. At the TeV energy scale, due to the presence of large Sudakov logarithms, EW corrections reach the 20-40\% level and play an important role for searches of physics beyond the Standard Model. The dependence of NLO EW effects on the jet multiplicity is investigated in detail, and we find that $W+$ multijet final states feature genuinely different EW effects as compared to the case of $W+1$ jet.

KEYwords: NLO Computations, Hadronic Colliders

ARXIV EPRINT: 1412.5157 


\section{Contents}

1 Introduction 1

2 General aspects of NLO electroweak corrections 4

2.1 Power counting in $\alpha$ and $\alpha_{\mathrm{S}}$

2.2 Virtual and real electroweak corrections 5

2.3 Photon-induced processes 6

2.4 Democratic jet clustering, quark fragmentation and photon recombination $\quad 7$

3 Automation of electroweak corrections in OpenLoops, Munich and Sherpa

$\begin{array}{lll}3.1 & \text { Tree and one-loop amplitudes with OpenLoops } & 10\end{array}$

3.2 Real radiation and QCD+QED subtraction with SHERPA and Munich 14

4 Electroweak and QCD corrections to $p p \rightarrow W^{+}+1,2,3$ jets 16

$\begin{array}{lll}4.1 & \text { Partonic channels } & 16\end{array}$

4.2 Two-quark contributions to $p p \rightarrow W^{+}+n$ jets 18

$\begin{array}{lll}4.3 & \text { Four-quark contributions to } p p \rightarrow W^{+}+n \text { jets } & 19\end{array}$

4.4 Photon-induced processes 21

4.5 Technical aspects of the on-shell approximation 22

5 Setup of the simulation $\quad 24$

6 NLO QCD+EW predictions for $W^{+}+1,2,3$ jets at the LHC 25

$\begin{array}{lll}6.1 W^{+}+1 \text { jet } & 27\end{array}$

$6.2 W^{+}+2$ jets 30

$6.3 W^{+}+3$ jets 34

6.4 Subleading and photon-induced Born contributions 35

$\begin{array}{llr}7 & \text { Summary and conclusions } & 39\end{array}$

\section{Introduction}

The production of a $W$ boson in association with jets represents one of the most prominent classes of processes at the Large Hadron Collider (LHC). Thanks to the high cross section and clean experimental signature, $W+$ jet production can be probed with high accuracy over a wide range of jet multiplicities and energy scales [1-6]. Such measurements provide a powerful testing ground for the Standard Model as well as for perturbative QCD methods and tools that build the fundament of all theoretical simulations of high-energy 
collisions at hadron colliders. The process $p p \rightarrow W+$ jets represents also an important background to various benchmark Standard Model reactions, such as $t \bar{t}$, single-top, diboson and Higgs-boson production. Moreover $W+$ multijet production is the dominant background in several searches of physics beyond the Standard Model (BSM) that are based on signatures with leptons, missing energy, and jets. In this context, precise theoretical predictions and reliable uncertainty estimates for the $W+$ multijet background can play a critical role for the precision of the measurements and the sensitivity to new phenomena. In particular, the accuracy of theoretical simulations of $W+$ multijet production at large transverse momentum and high jet multiplicity is very important for BSM searches at the TeV scale.

Predictions for $W+1 j$ and $W+2 j$ production at next-to-leading order (NLO) in QCD have been known for many years [7-17]. More recently, the advent of on-shell methods $[18,19]$ lead to the completion of NLO QCD calculations for $W+$ multijet production with three [20-23], four [24], and even five [25] associated jets. The inclusion of NLO QCD corrections strongly reduces the renormalisation and factorisation scale dependence of $W+$ multijet predictions, especially for high-multiplicity final states.

At NLO QCD, scale uncertainties for $W+$ multijet production are typically below $10 \%$ and can be regarded as a realistic estimate of the error due to missing NNLO QCD corrections. However, QCD scale variations do not reflect the uncertainty due to missing electroweak (EW) corrections. This is particularly relevant at high transverse momenta, where EW corrections are strongly enhanced by logarithmic contributions of Sudakov type [26-32], which can reach several tens of percent at the TeV scale. Electroweak NLO effects are thus the dominant source of theoretical uncertainty in NLO QCD simulations of $W+$ multijet production at high transverse momenta, and their inclusion can significantly improve the sensitivity to BSM searches at the energy frontier.

Electroweak NLO predictions for $W$-boson production in association with a single jet have been presented in $[33,34]$ for the case of stable $W$ bosons, and in [35] for the related process $p p \rightarrow \ell \nu j$, which includes resonant and non resonant contributions to $W \rightarrow \ell \nu$ decays. At high transverse momenta the EW corrections to $p p \rightarrow W+1 j$ are negative and very large. They reach about $-40 \%$ at $2 \mathrm{TeV}[33,34]$. The impact of NLO EW corrections on vector-boson plus multijet processes is expected to be similarly sizable. However, due to their higher technical complexity, NLO EW calculations for multijet final states are almost completely unexplored to date. The importance of EW Sudakov logarithms for the $Z+$ multijet background to Supersymmetry searches has been estimated in [36], using the next-to-leading logarithmic approximation of [28]. Very recently, using the automated one-loop generator RECola [37, 38], Denner et al. have presented NLO EW predictions for $p p \rightarrow \ell^{+} \ell^{-} j j[39,40]$, which represents the first NLO EW calculation for vector-boson production in association with more than one jet. Important steps towards the automation of NLO EW corrections have been undertaken also within the MADGRAPH5_aMC@NLO framework [41, 42] and by the GoSAm [43] collaboration.

In this paper we present a fully automated implementation of NLO EW corrections based on the OpenLoops one-loop generator [44] in combination with the $\mathrm{MUNICH}^{1}$ and

\footnotetext{
${ }^{1}$ MUNich is the abbreviation of "MUlti-chaNnel Integrator at Swiss (CH) precision" — an automated parton level NLO generator by S. Kallweit.
} 
Sherpa [45-47] Monte Carlo programs. The implemented algorithms are highly efficient and fully general. They support NLO QCD and EW simulations of high-energy collisions for any Standard Model process up to high particle multiplicity. As an application we consider $W+$ multijet production and, for the first time, we present NLO QCD+EW predictions for $p p \rightarrow W+2 j$ and $p p \rightarrow W+3 j$ at the LHC. Given that, at least for the case of $W+1 j$ production, the $\mathrm{EW}$ corrections feature a neglible dependence on the $W$-boson charge [34], in this paper we restrict ourselves to the case of positively charged $W$ bosons.

Virtual EW corrections are automated within the OpEnLOops framework, which is based on a fast numerical recursion for the generation of one-loop scattering amplitudes in the Standard Model [44]. The OpenLoops program has already been applied to various nontrivial NLO QCD [48-53] and NNLO QCD [54-57] simulations, ${ }^{2}$ and its first public version was released very recently. ${ }^{3}$ As compared to QCD corrections, in the EW sector virtual corrections are significantly more involved as they receive contributions from a wider set of particles $(\gamma, Z, W, H)$, which are characterised by a nontrivial mass spectrum. Moreover, while NLO QCD corrections are usually dominated by real-emission effects, in the case of NLO EW corrections the most prominent role is typically played by the one-loop virtual contributions. In particular, the exchange of virtual EW gauge bosons can give rise to large Sudakov logarithms.

Within our computational framework virtual EW corrections are complemented by two independent and fully automated implementations of NLO QED bremsstrahlung. The first one is based on Munich, a fully generic and very fast parton-level Monte Carlo integrator that has already been applied to various nontrivial multi-particle NLO calculations $[48,50$, 58-60] and also to NNLO calculations [55-57] based on $q_{\mathrm{T}}$-subtraction [61]. The second implementation of QED bremsstrahlung is based on the SHERPA Monte Carlo generator [46, 47], which was used in the pioneering NLO QCD calculations of vector-boson plus multijet production [20-25], as well as for their matching to the parton shower [62] and the merging of multijet final states at NLO [63]. Both Monte Carlo tools, MUNich and SHERPA, employ the dipole subtraction scheme $[64,65]$ for the cancellation of infrared singularities. The relevant one-loop and (in the case of $\mathrm{MUNICH}$ ) tree matrix elements are obtained from OpenLoops through generic built-in interfaces, and the full chain of operations that are relevant for NLO EW and QCD simulations - from process definition to the calculation of fully differential collider observables - is supported in a completely automated way. These tools have the potential to address NLO QCD+EW simulations for a very wide range of processes. As reflected in the 2013 Les Houches wish list [66], this represents one of the key priorities for the accurate theoretical interpretation of the data that will be collected during Run2 of the LHC.

The paper is organised as follows. Section 2 is devoted to general features of NLO EW corrections, including the interplay of NLO EW and QCD contributions, the treatment of initial- and final-state photons, and the real emission of weak gauge bosons. The automa-

\footnotetext{
${ }^{2}$ In the context of the NNLO calculations of [54-57] OpENLoOps was used for the evaluation of all relevant real-virtual and real-real amplitudes.

${ }^{3}$ The OpenLoops one-loop generator by F. Cascioli, J. Lindert, P. Maierhöfer and S. Pozzorini is publicly available at http://openloops.hepforge.org.
} 
tion of NLO QCD+EW simulations is presented in section 3, with emphasis on genuinely new aspects that go beyond a mere extension of NLO automation from the SU(3) to the $\mathrm{SU}(2) \times \mathrm{U}(1)$ sector of the Standard Model. The building blocks of the NLO QCD+EW calculation of $p p \rightarrow W+1,2,3$ jets are introduced in section 4 , where technical subtleties related to the on-shell treatment of $W$ bosons are discussed in detail. The setup of the simulation and numerical predictions for $W^{+}$production in association with up to three jets at the $13 \mathrm{TeV}$ LHC are presented in sections 5 and 6, respectively. The dependence of NLO EW effects on the jet multiplicity and new features that emerge in multijet final states are studied in detail. Our conclusions can be found in section 7 .

\section{General aspects of NLO electroweak corrections}

In this section we discuss general aspects of NLO EW calculations that play a nontrivial role in the definition of physical observables as well as for the extension of automated NLO algorithms from the QCD to the EW sector of the Standard Model.

\subsection{Power counting in $\alpha$ and $\alpha_{\mathrm{S}}$}

In the case of simple scattering processes, where the Born cross section can be associated with a unique perturbative order $\alpha_{\mathrm{S}}^{n} \alpha^{m}$ with fixed powers $m$ and $n$, the NLO QCD and EW corrections can be unambiguously identified as, respectively, the $\mathcal{O}\left(\alpha_{\mathrm{S}}^{n+1} \alpha^{m}\right)$ and $\mathcal{O}\left(\alpha_{\mathrm{S}}^{n} \alpha^{m+1}\right)$ contributions to the cross section. However, in general, scattering processes can receive various Born contributions of $\mathcal{O}\left(\alpha_{\mathrm{S}}^{n} \alpha^{m}\right)$ with $n+m$ fixed, and $0 \leq n, m \leq n+m$. In this case, which applies to processes that involve more than one external quark-antiquark pair, the naive separation of NLO QCD and NLO EW effects is not possible, and infrared singularities of QCD and EW type start "overlapping". This feature is schematically depicted in figures 1-2 for the case of $q \bar{q} \rightarrow q^{\prime} \bar{q}^{\prime}$ scattering, which is the simplest process with a nontrivial EW-QCD interplay. In general, at Born level it receives contributions ${ }^{4}$ of order $\alpha_{\mathrm{S}}^{2}, \alpha_{\mathrm{S}} \alpha$ and $\alpha^{2}$. The representative diagrams in figure 1 illustrate what might be naively regarded as the NLO EW correction to the $\mathcal{O}\left(\alpha_{\mathrm{S}}^{2}\right)$ Born contribution, namely terms of $\mathcal{O}\left(\alpha_{\mathrm{S}}^{2} \alpha\right)$ that result from order $\alpha_{\mathrm{S}} \times \alpha_{\mathrm{S}}$ tree interferences (figure 1a) via insertions of real photons (figure 1b) or virtual EW particles (figure 1c). However, as illustrated in figure 2, contributions of the same order $\alpha_{\mathrm{S}}^{2} \alpha$ can be obtained also from $\alpha_{\mathrm{S}} \times \alpha$ tree interferences (figure $2 \mathrm{a}$ ) via insertions of real (figure $2 \mathrm{~b}$ ) or virtual QCD partons (figure $2 \mathrm{c}$ ). The latter can be naively regarded as the NLO QCD corrections to the $\mathcal{O}\left(\alpha_{\mathrm{S}} \alpha\right)$ Born contribution. However, a consistent separation of $\mathcal{O}\left(\alpha_{\mathrm{S}}^{2} \alpha\right)$ corrections into NLO EW and NLO QCD terms, as suggested through figures 1-2, is not possible. First of all, the two categories overlap since diagrams like the one-loop topology in figure 1c can be regarded both as an EW or QCD correction to a gluon- or $\gamma / Z$-exchange tree amplitude, respectively. Moreover, this type of diagrams involves infrared (IR) singularities of EW and QCD type, whose cancellation requires photon and gluon emission terms of type $1 \mathrm{~b}$ and $2 \mathrm{~b}$, respectively. It is thus clear that the full set of contributions of $\mathcal{O}\left(\alpha_{\mathrm{S}}^{2} \alpha\right)$ must be taken into account. These

\footnotetext{
${ }^{4}$ Mixed interference terms of $\mathcal{O}\left(\alpha_{\mathrm{S}} \alpha\right)$ contribute only in case of equal quark flavours, $q^{\prime}=q$.
} 


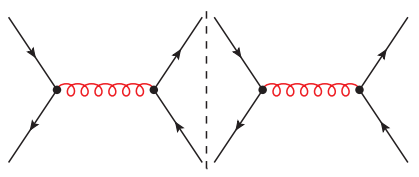

(a) Leading QCD Born.

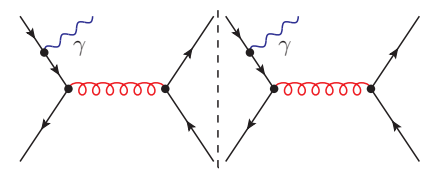

(b) Real $\mathcal{O}\left(\alpha_{\mathrm{S}}^{2} \alpha\right)$ correction.

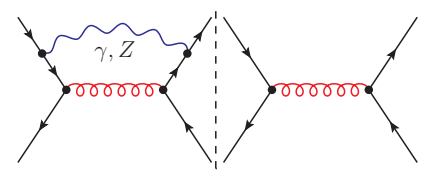

(c) Virtual $\mathcal{O}\left(\alpha_{\mathrm{S}}^{2} \alpha\right)$ correction.

Figure 1. Corrections of $\mathcal{O}\left(\alpha_{\mathrm{S}}^{2} \alpha\right)$ that are generated by dressing $\mathcal{O}\left(\alpha_{\mathrm{S}}^{2}\right)$ Born terms with real or virtual EW partons.

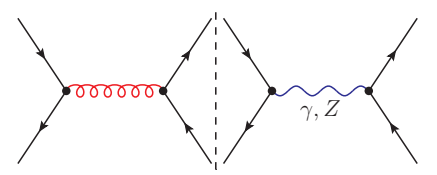

(a) QCD-EW Born interference.

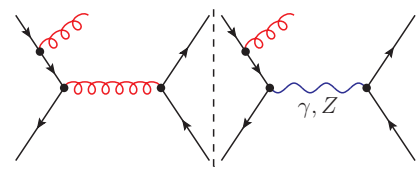

(b) Real $\mathcal{O}\left(\alpha_{\mathrm{S}}^{2} \alpha\right)$ correction.

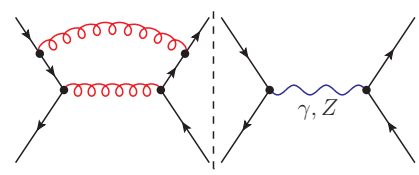

(c) Virtual $\mathcal{O}\left(\alpha_{\mathrm{S}}^{2} \alpha\right)$ correction.

Figure 2. Corrections of $\mathcal{O}\left(\alpha_{\mathrm{S}}^{2} \alpha\right)$ that are generated by dressing $\mathcal{O}\left(\alpha_{\mathrm{S}} \alpha\right)$ Born terms with real or virtual QCD partons. The interference of the tree diagrams in figure $2 \mathrm{a}$ vanishes as a result of their particular colour flow, but this picture should be understood as a schematic illustration of non-vanishing EW-QCD interferences that arise between $s$-channel and $t$-channel contributions to same-flavour $q \bar{q} \rightarrow q \bar{q}$ scattering, or in processes with additional external gluons.

considerations can be extended to processes involving additional external gluons, quarks and EW particles, and in general only the full set of contributions with a fixed order in $\alpha_{\mathrm{S}}$ and $\alpha$ can be considered as a well defined perturbative prediction. As far as the terminology is concerned, the most transparent approach is to label each contribution with the respective order in $\alpha_{\mathrm{S}}$ and $\alpha$. However, depending on the context, it might be convenient to denote the full set of $\mathcal{O}\left(\alpha_{\mathrm{S}}^{n} \alpha^{m+1}\right)$ terms as NLO EW correction with respect to $\mathcal{O}\left(\alpha_{\mathrm{S}}^{n} \alpha^{m}\right)$ or, alternatively, as NLO QCD correction with respect to $\mathcal{O}\left(\alpha_{\mathrm{S}}^{n-1} \alpha^{m+1}\right)$.

\subsection{Virtual and real electroweak corrections}

The infrared safe definition of physical observables requires the combination of virtual and real corrections at the same perturbative order. As discussed above, the cancellation of all virtual IR singularities at a certain order $\alpha_{S}^{n} \alpha^{m+1}$ can require various bremsstrahlung processes that involve additional photons, QED charged particles (quarks and leptons) and also QCD partons (gluons and quarks). The inclusion of such bremsstrahlung contributions is mandatory, since the emission of massless partons cannot be resolved as a separate process in the soft and collinear limits. As for the emission of heavy particles, i.e. $W, Z$ and Higgs bosons or top quarks, the situation is different. For instance, in QCD, from the viewpoint of $\alpha_{\mathrm{S}}$ power counting, top quark emissions can be included in the definition of NLO bremsstrahlung on the same footing as light-quark emissions. However, top-quark emissions are not indispensable for the cancellation of IR singularities, and since they lead to completely different experimental signatures, final states with additional top quarks are most conveniently handled as separate processes. For example, it is preferable to exclude $p p \rightarrow t \bar{t} W$ from the NLO QCD corrections to $p p \rightarrow W+1 j$, and to treat it as a separate $2 \rightarrow 3$ process. 
Similarly, at NLO EW, while the emission of heavy particles can be formally treated as NLO bremsstrahlung together with photon emission, we advocate a process bookkeeping approach where massive emissions are handled as separate processes, and only massless (or light) emissions are included in the definition of NLO EW corrections. For instance, $p p \rightarrow W Z$ should not be included in the NLO EW corrections to single $W$ production, and should be kept as a separate diboson production process. Of course, certain observables receive contributions both from $W Z$ and single $W$ final states, but the different physics dynamics of the two processes, which are individually IR finite, provides a strong motivation for a systematic separation of theoretical predictions for $p p \rightarrow W$ and $p p \rightarrow W Z$. Moreover, we point out that a systematic inclusion of massive EW bremsstrahlung at NLO can lead to quite unpleasant ambiguities and double counting issues. In particular, besides the overlap between processes with different vector boson multiplicity, such as $W$ and $W Z$ production, also processes involving different kinds of vector bosons would start overlapping. For example, $W Z$ production would contribute to the NLO EW corrections to both single $W$ and single $Z$ production.

Thus, in order to avoid overlap and double-counting issues, at the technical level it is preferable to adopt a process bookkeeping approach that keeps massive real emissions apart from the NLO EW corrections to the respective "no emission" processes. On the other hand, at the level of physical observables, one has to keep in mind that these two contributions enter at the same perturbative order and are related to each other in a subtle way. In particular, at the $\mathrm{TeV}$ scale both contributions involve large Sudakov logarithms, whose effects can partially cancel against each other in a way that bears some analogies with the cancellation of IR singularities in QCD. More precisely, at the TeV scale oneloop EW amplitudes involve large negative logarithms, which originate from the exchange of virtual $Z / W$ bosons in the soft and collinear regions and tend to be compensated by the real emission of soft and collinear $Z / W$ bosons [30-32]. However, for realistic collider processes this kind of cancellation is always incomplete and often rather modest. Firstly, Sudakov logarithms of soft origin do not cancel completely since initial- and final-state particles carry $\mathrm{SU}(2) \times \mathrm{U}(1)$ charges and thus do not fulfill the conditions of the BlochNordsieck theorem [30]. Secondly, Sudakov logarithms from initial-state collinear weakboson emission do not cancel at all, since they are not factorised into standard PDFs. Thirdly, the suppression of parton luminosities at high centre-of-mass energy and other kinematic effects tend to reduce the quantitative impact of the emission of extra heavy particles in a significant way. Finally, as far as differential observables and experimental cuts are concerned, one should keep in mind that the contributions from virtual and real $Z / W$ bosons behave in a completely different way.

In summary, in presence of large EW Sudakov effects the interplay between virtual EW corrections and massive EW bremsstrahlung deserves detailed quantitative studies, but these different contributions can and should be simulated as independent processes.

\subsection{Photon-induced processes}

Electroweak NLO corrections involve various types of massless real-emission contributions that arise from $q \rightarrow q \gamma, \bar{q} \rightarrow \bar{q} \gamma$, and $\gamma \rightarrow q \bar{q}$ splitting processes, as well as from analogous 
leptonic and usual QCD splittings. In the case of hadronic collisions, initial-state emissions of photons and quarks give rise to $\mathcal{O}(\alpha)$ collinear singularities that need to be factorised into the PDFs. This requires the introduction of a photon distribution function and the inclusion of QED effects in the DGLAP evolution of the (anti)quark and photon densities [67, 68]. Consequently, hadronic cross sections receive photon-induced contributions with photon-hadron and photon-photon initial states.

For what concerns the power counting in $\alpha$, one option is to treat the photon density as $\mathcal{O}(1)$ contribution, similarly as for the quark and gluon PDFs. In this case, for EW-induced processes such as dilepton and $W^{+} W^{-}$hadro-production, the $\gamma \gamma$ channel can contribute already at LO, and the corresponding NLO EW corrections involve $q \gamma$ and $\bar{q} \gamma$-induced bremsstrahlung contributions with an additional final state (anti)quark. In QCD-induced hadronic collisions where (anti)quark-gluon channels are open at LO, also (anti)quark-photon tree level channels contribute. However the latter involve a relative suppression factor $\alpha / \alpha_{\mathrm{S}}$. Similar considerations hold also for gluon-photon induced processes that involve $q \bar{q}$ pairs in the final state.

As an alternative power-counting approach, one can handle the photon PDF as an $\mathcal{O}(\alpha)$ contribution. This is justified by the fact that, in the typical kinematic range of LHC collisions, the ratio of the photon to gluon PDFs is of order $10^{-2}$. In this case, $\gamma$-hadron and $\gamma \gamma$-induced processes enter only at NLO and NNLO, respectively. Thus at NLO only tree level $\gamma$-hadron induced processes need to be included, if they contribute at all to the considered order in $\alpha_{\mathrm{S}}$ and $\alpha$. Such $\gamma$-hadron tree processes enter at the same perturbative order as NLO bremsstrahlung contributions associated with initial-state $q \rightarrow q \gamma^{*}$ and $\bar{q} \rightarrow \bar{q} \gamma^{*}$ splittings, thereby ensuring the consistent factorisation of the related collinear singularities into the photon PDF.

For particular processes and kinematic regions where $\gamma$-induced contributions turn out to be enhanced one should either include all NLO terms by counting the photon density as $\mathcal{O}(1)$ PDF, or stick to the $\mathcal{O}(\alpha)$ photon PDF approach and include those photon-induced contributions that are formally of NNLO in this counting scheme, but quantitatively important.

\subsection{Democratic jet clustering, quark fragmentation and photon recombination}

In order to guarantee the cancellation of infrared (soft and collinear) singularities in perturbative QCD, jet observables need to be defined through infrared-safe jet algorithms. In particular, jets must be insensitive to radiative processes that involve the emission of massless QCD partons in the soft and collinear limits, i.e. emission and no emission of soft or collinear partons must be indistinguishable at the level of jet observables. In presence of NLO EW corrections, it is clear that the requirement of IR safeness needs to be extended to the singularities associated with $q \rightarrow q \gamma, \bar{q} \rightarrow \bar{q} \gamma$ and $\gamma \rightarrow q \bar{q}$ QED splittings. In principle, this can be easily achieved through the so-called democratic jet clustering approach [6971], where photons and QCD partons are handled on the same footing at each clustering step. Jets resulting from democratic clustering contain photons, quarks and gluons, and their four-momenta are determined by the sum of all jet constituents, including photons. 
While the cancellation of collinear singularities of QCD and QED type is automatically ensured by democratic jet clustering, such a combined treatment of collinear quark-photon and gluon-photon pairs can hamper the cancellation of soft-gluon singularities. This is due to the fact that democratic jets are completely inclusive with respect to collinear photon emission, i.e. the photon energy fraction inside a jet, $z_{\gamma}=E_{\gamma} / E_{\text {jet }}$, extends over the whole range $0 \leq z_{\gamma} \leq 1$. This inclusiveness is crucial for the cancellation of collinear singularities associated with (anti)quark-photon pairs. However, in the case of gluon-photon pairs, in the region $z_{\gamma} \rightarrow 1$, where the jet consists of an almost pure photon, the gluon emission inside the jet becomes arbitrarily soft, thereby giving rise to IR QCD singularities.

The consistent cancellation of this kind of singularities can be achieved in two different ways. The first solution is to adopt a democratic treatment of photons and QCD partons also in the definition of processes that involve final-state jets. This implies that, at tree level, a jet can consist of either a QCD parton or a photon, while $N$-jet production receives tree level contributions from subprocesses with a variable number of final state QCD partons, $N_{g+q}$, and final state photons, $N_{\gamma}=N-N_{g+q}$, depending on the actual order $\alpha_{\mathrm{S}}^{n} \alpha^{m}$. In this approach, the related NLO EW photon bremsstrahlung at $\mathcal{O}\left(\alpha_{\mathrm{S}}^{n} \alpha^{m+1}\right)$ involves processes with $N_{\gamma}+1$ photons and $N_{g+q}$ final-state partons, and since photons count as jets, the requirement of $N$ hard jets does not guarantee that all $N_{g+q}$ partons are hard and well separated. In fact, the radiated photon can play the role of the $N^{\text {th }}$ jet, thereby allowing one of the QCD partons to become soft and/or collinear to a photon or to another parton. Nevertheless, in this approach, all resulting QCD singularities are cancelled by the virtual QCD corrections to the production of $N_{\gamma}+1$ photons plus $N_{g+q}-1$ QCD partons, which are automatically included in the democratic definition of $N$-jet final states.

Alternatively, one can adopt an approach aimed at preserving the distinction between QCD jets and photons, in such a way that processes with different numbers of QCD jets and photons do not mix. In this case, in order to avoid the soft QCD singularities that arise from jets with $z_{\gamma} \rightarrow 1$, the notion of QCD jets needs to be restricted to clusters of partons and photons where the photon-energy fraction does not exceed a certain threshold $z_{\text {thr }}<1$, while jets with $z_{\gamma}>z_{\text {thr }}$ have to be considered as photons. As for IR singularities of QED type, a strict implementation of the condition $z_{\gamma}<z_{\text {thr }}$ implies a fully exclusive description of collinear photon emissions off quarks, which hampers the cancellation of the related collinear singularity. A rigorous solution to this problem requires the factorisation of the collinear QED singularity in a non-perturbative quark-fragmentation function [40, 69, $72-$ 76]. However, as a pragmatic alternative to the fragmentation formalism, the cancellation of the collinear singularity can be enforced by recombining (anti)quark-photon pairs in a tiny cone around the singular region. As discussed in the following, this latter solution provides a quite reliable approximation to the rigorous fragmentation approach. Its algorithmic formulation, at NLO parton level, is as follows:

1. Collinear (anti)quark-photon pairs with rapidity-azimuthal separation $\Delta R_{\gamma, q} \leq$ $R_{\gamma q}^{\mathrm{rec}} \ll 1$ are recombined and are treated as (anti)quarks, so that collinear photons remain unresolvable in all subsequent steps of the algorithm.

2. A jet-clustering algorithm is applied, where photons and QCD partons are treated on equal footing at each recombination step. 
3. Jets that contain resolvable photons, i.e. photons that have not been recombined in step 1, are considered as QCD jets only if the photon-energy fraction $z_{\gamma}=E_{\gamma} / E_{\text {jet }}$ does not exceed a certain threshold $z_{\mathrm{thr}}<1$.

Is is clear that step 1 ensures the cancellation of collinear QED singularities. At the same time, the fact that the condition $z_{\gamma}<z_{\text {thr }}$ is not applied to recombined photons represents an approximate treatment of step 3. Since this approximation is restricted to a tiny cone around the collinear region, its quality can be easily assessed in a process independent way. To this end, let us consider a collinear $q \rightarrow q \gamma$ splitting, where a quark with transverse momentum $p_{\mathrm{T}}$ gives rise to a photon and a quark with momenta $z_{\gamma} p_{\mathrm{T}}$ and $\left(1-z_{\gamma}\right) p_{\mathrm{T}}$, respectively. Combining the perturbative contribution associated with the splitting function $P_{q \gamma}(z)=\left[1+(1-z)^{2}\right] / z$ with the non-perturbative fragmentation function extracted from ALEPH data $[72,76]$ leads to the following expression for the probability to find a photon with energy fraction $z_{\gamma}>z_{\text {thr }}$ within a cone of radius $R$ [34]:

$$
\epsilon_{q}\left(z_{\mathrm{thr}}, R, p_{\mathrm{T}}\right)=\int_{z_{\mathrm{thr}}}^{1} \mathrm{~d} z \mathcal{D}_{q \gamma}\left(z, R, p_{\mathrm{T}}\right)
$$

with

$$
\mathcal{D}_{q \gamma}\left(z, R, p_{\mathrm{T}}\right)=\frac{\alpha Q_{q}^{2}}{2 \pi}\left[2 P_{q \gamma}(z) \ln \left(\frac{z R p_{\mathrm{T}}}{\mu_{0}}\right)+z-C\right]
$$

where $Q_{q}$ is the electromagnetic charge of the quark, while the scale $\mu_{0}=0.14 \mathrm{GeV}$ and the parameter $C=13.26$ enter through the fit of the fragmentation component to ALEPH data. This quantity corresponds to the probability that a photon-like jet is misinterpreted as a QCD jet due to the photon-quark recombination prescription, i.e. it represents the relative uncertainty inherent in the first step of the above jet definition 1-3. Its quantitative impact in the case of up-type quarks is illustrated in figure 3 for a wide range of photon-energy thresholds and jet transverse momenta. For realistic threshold values $z_{\mathrm{thr}} \geq 0.5$, it is clear that the error induced by the recombination prescription is at the permil level. Moreover, in realistic jet-production processes this error is further suppressed since the treatment of gluon-photon pairs is exact, while for down-quark-photon pairs eq. (2.2) involves a smaller charge factor, $Q_{d}^{2}=Q_{u}^{2} / 4$. We thus conclude that the error inherent in the above recombination prescription can hardly exceed the few permil level in a very broad kinematic range.

\section{Automation of electroweak corrections in OpenLoops, Munich and Sherpa}

In this section we discuss the fully automated implementation of NLO QCD+EW corrections in OpenLoops [44], Munich and Sherpa [45-47]. In this computing framework, the OpenLoops program generates the relevant one-loop and, if needed, tree matrix elements, while the Munich and SHerpa Monte Carlo programs take care of all complementary NLO tasks, i.e. the bookkeeping of partonic processes, the subtraction of IR singularities, and phase-space integration. For what concerns Born and real-emission matrix elements, 


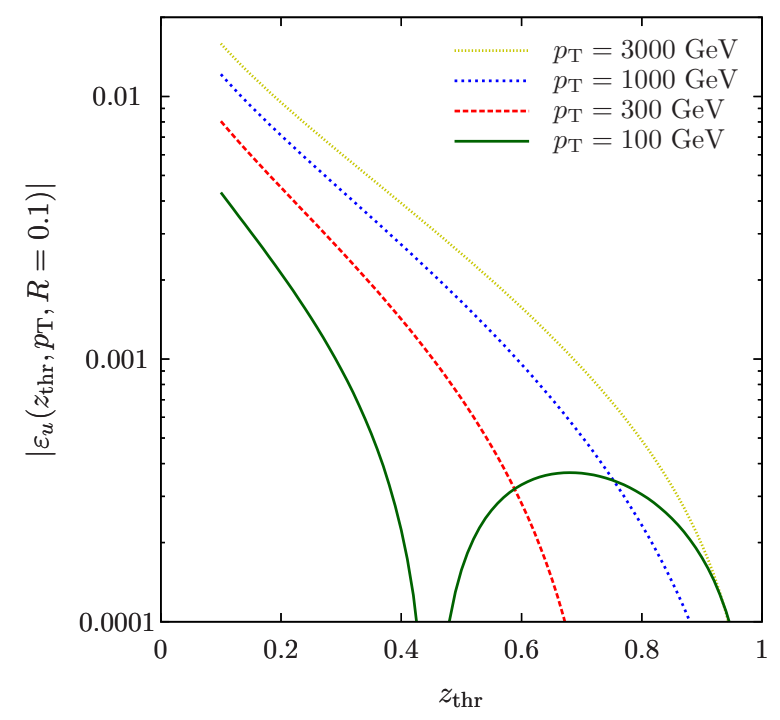

Figure 3. Probability $\epsilon_{u}\left(z_{\mathrm{thr}}, p_{\mathrm{T}}, R\right)$ of $u \rightarrow u \gamma$ fragmentation in a cone of radius $R=0.1$ as a function of the photon-energy threshold $z_{\text {thr }}$ for different values of the jet transverse momentum, $p_{\mathrm{T}}=100,300,1000,3000 \mathrm{GeV}$.

in Sherpa they are provided by the two internal tree-level generators Amegic $++[77]$ and Comix [78], while Munich takes them from OpenLoops. The present implementation supports parton-level NLO QCD+EW simulations in a fully automated way, and any hadron-collider observable can be generated in a few simple steps upon specification of the desired hadronic process and the relevant input parameters. In the following we will focus our attention on nontrivial aspects that had to be addressed in order to extend the functionality of the various tools from NLO QCD to NLO EW. The automation of NLO EW calculations will be available in future public releases of OpenLoops, Munich and SHerpa.

\subsection{Tree and one-loop amplitudes with OpenLoops}

The OpenLoops program is a fully automated generator of tree and one-loop scattering amplitudes within the Standard Model. Matrix elements are built with a recursive numerical algorithm [44], which is flexibly applicable to any desired process and guarantees high CPU performance up to high particle multiplicity. The first public version of OpEnLoops was released very recently. It supports NLO QCD calculations for a wide range of processes up to four final-state particles. The code is available as a set of compact libraries that cover more than one hundred different processes at hadron colliders, and the number of supported processes is continuously growing. The various process libraries contain all relevant ingredients for NLO QCD calculations: tree amplitudes, renormalised one-loop amplitudes, and colour- and helicity-correlated matrix elements for the subtraction of IR singularities. OpenLoops provides easy to use ForTRAn and $\mathrm{C}++$ interfaces, as well as a standard interface based on the Binoth Les Houches Accord [79], and can therefore be easily integrated within any Monte Carlo framework. Moreover, Sherpa [47] and HerwiG's MatchBox [80] as well as Munich dispose of generic built-in interfaces to OpenLoops. 


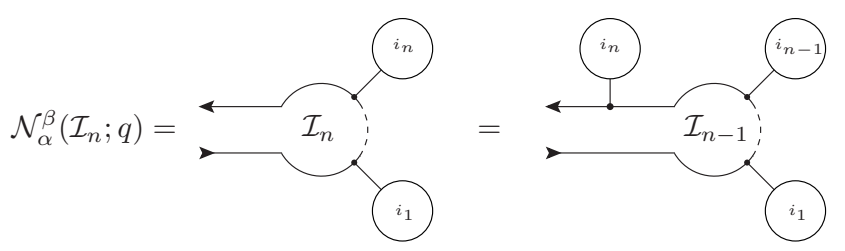

Figure 4. Schematic representation of the open-loops recursion: $n$-point open loops are constructed by merging $(n-1)$-point open loops and external subtrees.

In OpenLoops tree and one-loop amplitudes are computed in terms of individual colour-stripped Feynman diagrams. While the reduction of colour factors, colour interferences and colour sums are performed with algebraic techniques, the construction of colourstripped diagrams is entirely numerical. The tree algorithm is based on subtrees, which correspond to pieces of individual colour stripped tree diagrams that result from cutting an internal propagator. Tree amplitudes are generated via recursive merging of subtrees, and the systematic exploitation of relations between diagrams that share common subtrees allows one to evaluate multi-particle amplitudes with high CPU efficiency.

One-loop amplitudes in OpenLoops are constructed by means of a hybrid tree-loop recursion that generates cut-open loops as functions of the circulating loop momentum [44]. The basic building blocks are individual colour-stripped one-loop diagrams of the form

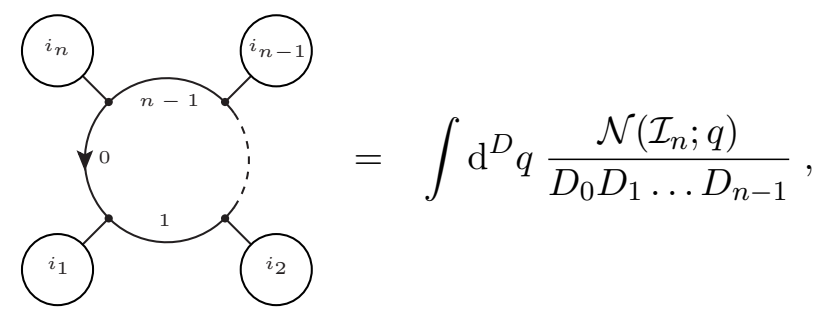

where $D_{i}=\left(p_{i}-q\right)^{2}-m_{i}^{2}+\mathrm{i} \epsilon$, the blobs $i_{1}, \ldots, i_{n}$ represent external subtrees, and the numerator $\mathcal{N}\left(\mathcal{I}_{n} ; q\right)$ is a polynomial in the loop momentum $q$. Cut-opening the internal line associated with the $D_{0}$ propagator, converts the loop into a tree structure and promotes the numerator to a tensor, $\mathcal{N}_{\alpha}^{\beta}(\mathcal{I} ; q)$, whose two indices are associated with the spin or vector degrees of freedom of the cut propagator. As sketched in figure 4, these objects can be constructed in a similar way as tree amplitudes, by recursively merging the external subtrees that are attached to the loop. Formally, this corresponds to the recurrence relation

$$
\mathcal{N}_{\alpha}^{\beta}\left(\mathcal{I}_{n} ; q\right)=X_{\gamma \delta}^{\beta}\left(\mathcal{I}_{n}, i_{n}, \mathcal{I}_{n-1}\right) \mathcal{N}_{\alpha}^{\gamma}\left(\mathcal{I}_{n-1} ; q\right) w^{\delta}\left(i_{n}\right),
$$

where $w^{\delta}\left(i_{n}\right)$ represents the $n$-th external subtree, while the tensor $X_{\gamma \delta}^{\beta}$, which describes the interaction of the $i_{n}$-th subtree with the rest of the cut-open diagram, depends only on the flavour and the momenta of the involved particles in a way that is dictated by the Feynman rules of the theory. In contrast to conventional tree algorithms of type (3.2), in OpenLoops all ingredients are handled as polynomials in the circulating loop momentum. The numerator assumes the form

$$
\mathcal{N}_{\alpha}^{\beta}\left(\mathcal{I}_{n} ; q\right)=\sum_{r=0}^{R} \mathcal{N}_{\mu_{1} \ldots \mu_{r} ; \alpha}^{\beta}\left(\mathcal{I}_{n}\right) q^{\mu_{1}} \ldots q^{\mu_{r}},
$$


where $R \leq n$ is the maximum rank of tensor integrals that contribute to the actual loop diagram, while the interaction term is expressed as ${ }^{5}$

$$
X_{\gamma \delta}^{\beta}=Y_{\gamma \delta}^{\beta}+q^{\nu} Z_{\nu ; \gamma \delta}^{\beta} .
$$

The one-loop algorithm is formulated as a recurrence relation for the direct construction of the $q$-polynomial coefficients:

$$
\mathcal{N}_{\mu_{1} \ldots \mu_{r} ; \alpha}^{\beta}\left(\mathcal{I}_{n}\right)=\left[Y_{\gamma \delta}^{\beta} \mathcal{N}_{\mu_{1} \ldots \mu_{r} ; \alpha}^{\gamma}\left(\mathcal{I}_{n-1}\right)+Z_{\mu_{1} ; \gamma \delta}^{\beta} \mathcal{N}_{\mu_{2} \ldots \mu_{r} ; \alpha}^{\gamma}\left(\mathcal{I}_{n-1}\right)\right] w^{\delta}\left(i_{n}\right) .
$$

This type of algorithm was originally proposed in the framework of a Dyson-Schwinger recursion for colour-ordered gluon-scattering amplitudes [81]. The fact that loop numerators are directly constructed as functions of the loop momentum represents a great advantage for the speed of the algorithm. The actual implementation of (3.5) in OpenLoops employs fully symmetrised tensors. Its CPU efficiency is further augmented by means of parent-child relations and thanks to further tricks that exploit the systematic factorisation of colour-, helicity-, and q-dependent objects [44].

In order to extend OpenLoops to EW one-loop corrections, all EW Feynman rules for fermions, vector bosons, scalars and ghosts have been implemented in the form of numerical routines corresponding to the generic recursion relation (3.5). Each interaction term described by (3.4) is associated with three lines ${ }^{6}$ that play different roles: external subtree, inflowing and outflowing loop line. Thus, in general, each vertex in the Feynman rules requires three numerical routines of type (3.5). Once implemented, these universal routines are applicable to any one-loop amplitude within the QCD+EW Standard Model. Moreover, they can be easily extended to BSM interactions.

The numerical polynomial representation (3.3) of loop numerators provides full information on the functional $q$-dependence of the integrand, thereby allowing for great flexibility in the reduction of (3.1) to scalar integrals. On the one hand, the reduction can be performed at the level of individual tensor integrals associated with the monomials $q^{\mu_{1}} \ldots q^{\mu_{r}}$ in (3.3). To this end, OpenLoops is interfaced with the Collier library [82], which implements the Denner-Dittmaier reduction techniques $[83,84]$ and the scalar integrals of [85]. Sophisticated analytic expansions [83, 84] render this approach very robust against numerical instabilities in exceptional phase-space regions. Alternatively, the reduction of (3.1) to scalar integrals can be performed at the integrand level using the OPP method [86] as implemented in CutTools [87] or SAmurai [88], which both rely on the ONELOOP library [89] for the evaluation of scalar integrals.

The evaluation of one-loop QCD amplitudes with OpenLoops is very fast [44], both in combination with tensor integral reduction and OPP reduction. In this context it was observed that CPU timings grow only linearly with the number of Feynman diagrams, which guarantees a fairly favourable scaling with the external-particle multiplicity. We find

\footnotetext{
${ }^{5}$ Here we restrict ourselves to a linear $q$-dependence, assuming renormalisable interactions, but the generalisation to an arbitrary polynomial degree is straightforward. Also the formulation of quartic and higher-point interactions is obvious.

${ }^{6}$ In the case of quartic vertices there is a fourth line that enters as additional external wave function.
} 
that this property holds also for one-loop EW calculations. More precisely, the dependence of CPU timings on the number of Feynman diagrams per process is roughly universal, i.e. approximately the same for QCD and EW corrections.

Within OpenLoops, ultraviolet (UV) and infrared (IR) divergences are dimensionally regularised and take the form of poles in $(4-D)$. However, all ingredients of the numerical recursion (3.3)-(3.5) are handled in four space-time dimensions. The missing $(4-D)$ dimensional contributions - called $R_{2}$ rational terms - are universal and can be restored from process-independent effective counterterms [90-92]. Corresponding Feynman rules have been derived for QED in [90], for QCD in [93] and for the complete EW Standard Model in [94-97]. We implemented all QCD and EW $R_{2}$ counterterms in OpENLoOPS and validated them against independent algebraic results in $D=4-2 \epsilon$ dimensions.

For the renormalisation of UV divergences we adopted the on-shell scheme [98] and implemented all relevant $\mathcal{O}(\alpha)$ counterterm Feynman rules and related renormalisation constants for the full Standard Model, including the option of the complex mass scheme [99] for unstable gauge bosons and top quarks. In NLO QCD calculations the strong coupling constant is renormalised in the $\overline{\mathrm{MS}}$ scheme, and heavy quark contributions can be decoupled in a flexible way, depending on the number of active flavours in the evolution of $\alpha_{S}$. For the renormalisation of the electroweak couplings we implemented the $G_{\mu}$ scheme, where the fine-structure constant $\alpha=e^{2} / 4 \pi$ and the weak mixing angle $\theta_{\mathrm{w}}$ are given by

$$
\alpha=\frac{\sqrt{2}}{\pi} G_{\mu} M_{\mathrm{W}}^{2}\left(1-\frac{M_{\mathrm{W}}^{2}}{M_{\mathrm{Z}}^{2}}\right), \quad \cos \theta_{\mathrm{w}}=\frac{M_{W}}{M_{Z}}
$$

This requires a redefinition of the renormalisation constant associated with the electromagnetic coupling,

$$
\left.\delta Z_{e}\right|_{G_{\mu}}=\left.\delta Z_{e}\right|_{\alpha(0)}-\frac{1}{2} \Delta r
$$

where $\Delta r$ is defined in [98], and $\alpha(0)$ denotes the standard on-shell renormalisation prescription in the Thompson limit.

For the cancellation of the remaining IR singularities in the virtual QCD and EW corrections, OpEnLOOPS provides dedicated routines that implement the so-called $I$-operator in the dipole subtraction formalism [64, 65] and its extension to QED corrections [100-102]. In this context also colour-correlated and charge-correlated Born matrix elements at any desired order in $\alpha$ and $\alpha_{\mathrm{S}}$ are supported. Their content can be schematically represented as

$$
\begin{gathered}
\left.\left.g_{\mathrm{S}}^{2}\left\langle M_{0}\left|T^{a}(i) T^{a}(j)\right| M_{0}\right\rangle\right|_{\alpha_{\mathrm{S}}^{n+1} \alpha^{m}}=g_{\mathrm{S}}^{2} \sum_{p, p^{\prime}, q, q^{\prime}}\left\langle M_{0}^{(p, q)}\left|T^{a}(i) T^{a}(j)\right| M_{0}^{\left(p^{\prime}, q^{\prime}\right)}\right\rangle \delta_{2 n, p+p^{\prime}} \delta_{2 m, q+q^{\prime}},(3 .)^{\left(p^{\prime}, q^{\prime}\right)}\right\rangle \delta_{2 n, p+p^{\prime}} \delta_{2 m, q+q^{\prime}} \\
\left.e^{2}\left\langle M_{0}|Q(i) Q(j)| M_{0}\right\rangle\right|_{\alpha_{\mathrm{S}}^{n} \alpha^{m+1}}=e^{2} \sum_{p, p^{\prime}, q, q^{\prime}}\left\langle M_{0}^{(p, q)}|Q(i) Q(j)| M_{0}^{\left(p^{\prime}\right.}\right.
\end{gathered}
$$

where $T^{a}(i)$ denotes the usual colour-insertion operator acting on the $i^{\text {th }}$ external leg, and $Q(i)$ is the corresponding electromagnetic charge operator. The usual bra-ket notation is used for Born matrix elements and their complex conjugates, and sums over external-leg colours are implicitly understood. Born matrix elements of $\mathcal{O}\left(g_{\mathrm{S}}^{p} e^{q}\right)$ are denoted as 
$M_{0}^{(p, q)}$, and all relevant contributions to a predefined overall order are included in a fully automated way. Furthermore, OPENLOOPS provides extra routines to calculate gluon- and photon-helicity correlated Born amplitudes, which are needed by Monte Carlo programs to construct IR subtraction terms for real-emission matrix elements.

As far as the bookkeeping of the perturbative orders in $\alpha_{\mathrm{S}}$ and $\alpha$ is concerned, all relevant LO and NLO virtual contributions are generated and combined in a similar way as in (3.8)-(3.8), i.e. the following colour-summed Born-Born and Born-virtual interference terms that contribute to a given order are automatically combined,

$$
\begin{aligned}
\left.\left\langle M_{0} \mid M_{0}\right\rangle\right|_{\alpha_{\mathrm{S}}^{n} \alpha^{m}} & =\sum_{p, p^{\prime}, q, q^{\prime}}\left\langle M_{0}^{(p, q)} \mid M_{0}^{\left(p^{\prime}, q^{\prime}\right)}\right\rangle \delta_{2 n, p+p^{\prime}} \delta_{2 m, q+q^{\prime}}, \\
\left.\left\langle M_{0} \mid M_{1}\right\rangle\right|_{\alpha_{\mathrm{S}}^{n} \alpha^{m}} & =\sum_{p, p^{\prime}, q, q^{\prime}}\left\langle M_{0}^{(p, q)} \mid M_{1}^{\left(p^{\prime}, q^{\prime}\right)}\right\rangle \delta_{2 n, p+p^{\prime}} \delta_{2 m, q+q^{\prime}} .
\end{aligned}
$$

Here, the inclusion of all counterterm contributions of UV and $R_{2}$ kind is implicitly understood. All nontrivial EW-QCD interference contributions described in section 2.1 are thus automatically taken into account. From the user viewpoint, specifying the desired order $\alpha_{\mathrm{S}}^{n} \alpha^{m}$ at LO and the type of correction, NLO QCD or NLO EW, is sufficient in order to obtain all relevant NLO terms of $\mathcal{O}\left(\alpha_{\mathrm{S}}^{n+1} \alpha^{m}\right)$ or $\mathcal{O}\left(\alpha_{\mathrm{S}}^{n} \alpha^{m+1}\right)$, respectively. Also the calculation of the complete NLO Standard Model corrections, including all relevant contributions of $\mathcal{O}\left(\alpha_{\mathrm{S}}^{n-k+1} \alpha^{m+k}\right)$ with $-m \leq k \leq n+1$ is possible. This flexible power counting is fully supported by the available OpENLOOPS interface.

The entire implementation of NLO EW virtual contributions in OpenLoops, including the finite parts of the UV renormalisation, has been checked for several processes. To this end we implemented NLO EW corrections in a second and fully independent in-house generator, which was originally developed for NLO QCD calculations [59, 103]. Detailed checks have been performed for all building blocks that enter the NLO QCD+EW corrections for $W$-boson production in association with jets presented in this paper.

\subsection{Real radiation and QCD+QED subtraction with Sherpa and Munich}

This section deals with the automated calculation of real-emission contributions at NLO QCD+EW level in Munich and SherPA. In this context, the first key task is the fully automated bookkeeping of the real-emission channels that contribute to any user-defined process with a certain number of jets, photons, leptons and additional heavy particles at Born level. More precisely, the programs generate the full list of contributing partonic processes organised according to their orders in $\alpha_{\mathrm{S}}$ and $\alpha$, together with the ones that involve one extra massless object in the final state, i.e. an extra gluon, a quark pair instead of a gluon, an extra photon, or a fermion pair instead of a photon. As discussed in section 2.4, jets and photons can be handled on the same footing or as separate physics objects, and the list of contributing subprocesses depends on the details of the photon/jet definition. ${ }^{7}$ However, the process bookkeeping can adapt to the above two options in a fully flexible way.

\footnotetext{
${ }^{7}$ Note that section 2.4 deals only with the infrared-safe definition of jets in processes with hard jets and no resolved photons, while the issue of IR safeness for processes with resolved photons at NLO QCD+EW is not addressed in this paper.
} 
In connection with the generation of the real radiation the main task of MUNICH and SHERPA is the consistent subtraction of IR singularities. To this end, both programs implement the Catani-Seymour formalism [64,65]. Light quarks and leptons are treated as massless particles, and the related singularities are regularised in $D$ dimensions. All relevant subtraction terms in the real-emission phase space are obtained from the convolution of QCD and QED Catani-Seymour splitting kernels with reduced Born contributions. Their integrated counterparts factorise into reduced Born matrix elements times the so-called $I, K$, and $P$ operators $[64,65]$. In this context, starting from existing implementations of dipole subtraction at NLO QCD, all process-independent building blocks, i.e. splitting kernels and $I+K+P$ operators, have been extended to NLO QCD+QED. ${ }^{8}$ In particular, all contributions associated with $f \rightarrow f \gamma, \bar{f} \rightarrow \bar{f} \gamma$, and $\gamma \rightarrow f \bar{f}$ QED splittings can be obtained from the related QCD contributions by applying the substitutions

$$
\alpha_{s} \longrightarrow \alpha, \quad C_{F} \longrightarrow Q_{f}^{2}, \quad T_{R} \longrightarrow N_{c, f} Q_{f}^{2}, \quad T_{R} N_{f} \longrightarrow \sum_{f} N_{c, f} Q_{f}^{2}, \quad C_{A} \longrightarrow 0,
$$

and the following additional replacements for the colour-correlation operators associated with an emitter $i j$ and a spectator $k$,

$$
\frac{\mathbf{T}_{i j} \cdot \mathbf{T}_{k}}{\mathbf{T}_{i j}^{2}} \longrightarrow\left\{\begin{array}{ll}
\frac{Q_{i j} Q_{k}}{Q_{i j}^{2}} & \text { if the emitter } i j \text { is a (anti)fermion } \\
\kappa_{i j, k} & \text { if the emitter } i j \text { is a photon, }
\end{array} \quad \text { with } \sum_{k \neq i j} \kappa_{i j, k}=-1\right.
$$

In practice, for the case of a photon emitter, one can restrict oneself to a single spectator particle $e_{i j}$ different from the fermion-antifermion emitter $i j$, i.e. $\kappa_{i j, k}=-\delta_{e_{i j}, k}$. Alternatively any sum over spectators different from $i j$ can be chosen as long as the last constraint in (3.13) is fullfilled. While the colour-insertion operators are reduced to multiplicative scalars in (3.13), the spin correlators of the real-subtraction terms associated with $\gamma \rightarrow f \bar{f}$ splittings preserve the same form as for $g \rightarrow q \bar{q}$ splittings in QCD.

Besides singularities of pure QED type, processes with external on-shell $W$ bosons involve additional singularities associated with $W \rightarrow W \gamma$ splittings. In this case, due to the large $W$-boson mass, no collinear singularity or logarithmic enhancement is present, and only the soft-photon singularity has to be subtracted. Exploiting the universal nature of soft singularities, in this publication this is achieved by using the heavy-fermion or heavy-scalar splitting function of [65], and, after the replacements of (3.12), identifying the heavy particle with the external $W$ boson.

As discussed in section 2.1, NLO QCD and EW corrections have to be understood, respectively, as the full set of $\mathcal{O}\left(\alpha_{\mathrm{S}}\right)$ and $\mathcal{O}(\alpha)$ corrections relative to a certain tree-level order $\alpha_{S}^{n} \alpha^{m}$. Moreover, in general, NLO QCD and EW corrections are not uniquely associated with the emission of corresponding (strongly or electroweakly interacting) particles. Actually, given a certain correction order, $\alpha_{\mathrm{S}}^{n+1} \alpha^{m}$ or $\alpha_{\mathrm{S}}^{n} \alpha^{m+1}$, each of the contributing real-emission processes can comprise various types of unresolved massless particles (gluons, photons, quark or lepton pairs) and IR singularities. In particular, NLO QCD (EW) corrections can involve singularities associated with both order $\alpha_{\mathrm{S}}(\alpha)$ splittings times order

\footnotetext{
${ }^{8}$ The construction of QED dipole-subtraction terms has been discussed in refs. [100-102].
} 
$\alpha_{\mathrm{S}}^{n} \alpha^{m}$ Born terms, and with order $\alpha\left(\alpha_{\mathrm{S}}\right)$ splittings times order $\alpha_{\mathrm{S}}^{n+1} \alpha^{m-1}\left(\alpha_{\mathrm{S}}^{n-1} \alpha^{m+1}\right)$ Born terms. Therefore, Munich and SHERPA implement a fully general bookkeeping of perturbative orders and singularities. The relevant dipole terms, to account for all possible QCD and QED splittings in a generic real-correction process, are selected in a fully automated way. Inevitably, the associated reduced Born matrix elements are allowed to be at a different order than the original Born configuration. For the integrated subtraction terms, a similarly general bookkeeping is applied, where all relevant QED and QCD contributions to the $I+K+P$ operators are combined with factorised Born matrix elements at the appropriate orders in $\alpha$ and $\alpha_{\mathrm{S}}$. This requires nontrivial combinations of charge/colour insertion operators and interferences of Born amplitudes at different orders, similarly as in (3.8)-(3.8).

For phase-space integration, both MUNICH and SHERPA employ adaptive multi-channel techniques. In SHERPA, dipole subtraction terms can be restricted by means of the so-called $\alpha$-dipole parameter [104-109], while MUNICH constructs extra phase-space mappings based on the dipole kinematics, and automatically adds them to the generic set of the realemission based phase-space parametrisations used in the multi-channel approach.

The SHERPA and Munich implementations have been validated with standard selfconsistency checks, such as the local cancellation of singularities in the real-emission phase space, the cancellation of the $\alpha$-dipole dependence in SHERPA and the equivalence of fermion and scalar splitting kernels for the subtraction of $W \rightarrow W \gamma$ soft singularities.

All involved colour-, charge- and spin-correlated matrix elements are provided by the OpenLoops generator in case of MUNICH, whereas they are supplied by AMEGIC ++ and Comix within the SHerpa implementation. Apart from the contributions that involve charge/colour insertions of type (3.8), which are still under construction within SHERPA, for all other building blocks the two programs have been validated against each other on a point-wise basis as well as for integrated cross sections for a wide range of processes, giving rise to full agreement on the level of machine precision and statistical precision, respectively. The point-wise agreement for the $I$-operator provided by OpenLoops, Munich and SHERPA was also checked. The results presented in section 6 have been obtained with Munich+OPENLOOPS.

\section{Electroweak and QCD corrections to $p p \rightarrow W^{+}+1,2,3$ jets}

To demonstrate the flexibility and the performance of NLO automation in OpENLoops together with SHERPA and MUNICH, as a first application we consider the NLO QCD+EW corrections to $W$-boson production in association with up to three jets at the LHC. In this paper we focus on the production of stable $W^{+}$bosons, while the case of $W^{-}$production as well as $W$-boson decays will be addressed in a subsequent publication. In the following we discuss the building blocks of our calculation and technical subtleties related to the on-shell treatment of final-state $W$ bosons at NLO EW.

\subsection{Partonic channels}

The level of automation of the employed tools is such that, to generate and evaluate all relevant contributions to a desired hadronic cross section, it is sufficient to specify the 
desired final state and the perturbative order in $\alpha_{\mathrm{S}}$ and $\alpha$. Thus, from the user viewpoint, there is no need to worry about the detailed content of the simulation in terms of partonic channels, scattering amplitudes and Feynman diagrams. Nevertheless, a basic knowledge of these ingredients plays an important role for the understanding of the physics content of the simulation and for the interpretation of the phenomenological results.

At tree level, the only crossing-independent partonic process that contributes to $p p \rightarrow$ $W^{+} j$ is

$$
u_{i} \bar{d}_{i} \rightarrow W^{+} g,
$$

where $u_{i}=(u, c)$ and $d_{i}=(d, s)$. All other relevant channels can be obtained from (4.1) through permutations of initial- and final-state partons. For $p p \rightarrow W^{+} 2 j$ there are two crossing-independent subprocesses:

$$
\begin{aligned}
& u_{i} \bar{d}_{i} \rightarrow W^{+} q \bar{q}, \\
& u_{i} \bar{d}_{i} \rightarrow W^{+} g g,
\end{aligned}
$$

and the relevant crossing-independent subprocesses for $p p \rightarrow W^{+} 3 j$ are obtained form (4.2) and (4.3) by adding an extra gluon:

$$
\begin{aligned}
& u_{i} \bar{d}_{i} \rightarrow W^{+} q \bar{q} g, \\
& u_{i} \bar{d}_{i} \rightarrow W^{+} g g g .
\end{aligned}
$$

The above processes can be categorised into two-quark and four-quark channels, according to the total number of external (anti)quarks. In the case of the four-quark channels, (4.2) and (4.4), the additional $q \bar{q}$ system can consist of any light-quark pair with $q \in\{u, d, s, c, b\}$. All light quarks are treated as massless particles in our calculation.

The main focus of this paper is on the NLO QCD and NLO EW corrections with respect to the dominant $\mathcal{O}\left(\alpha_{\mathrm{S}}^{n} \alpha\right)$ tree-level contributions to $p p \rightarrow W^{+}+n$ jets. With other words we will consider NLO contributions of $\mathcal{O}\left(\alpha_{\mathrm{S}}^{n+1} \alpha\right)$ and $\mathcal{O}\left(\alpha_{\mathrm{S}}^{n} \alpha^{2}\right)$, respectively. In both cases, $W^{+}+n$-jet production receives NLO bremsstrahlung contributions from treelevel amplitudes involving an extra parton. The relevant partonic channels are obtained from (4.1)-(4.5) either by replacing an external gluon by a $q \bar{q}$ pair, or by adding and external gluon or an external photon. At Born level, in the following we will discuss also mixed EW-QCD contributions of $\mathcal{O}\left(\alpha_{\mathrm{S}}^{n-1} \alpha^{2}\right)$, pure EW contributions of $\mathcal{O}\left(\alpha_{\mathrm{S}}^{n-2} \alpha^{3}\right)$, the tower of photon-proton induced contributions of $\mathcal{O}\left(\alpha_{\mathrm{S}}^{n-1} \alpha^{2}\right), \mathcal{O}\left(\alpha_{\mathrm{S}}^{n-2} \alpha^{3}\right)$ and $\mathcal{O}\left(\alpha_{\mathrm{S}}^{n-3} \alpha^{4}\right)$, and photon-photon induced contributions of $\mathcal{O}\left(\alpha_{\mathrm{S}}^{n-2} \alpha^{3}\right)$.

Table 1 summarises the number of $\mathcal{O}\left(\alpha_{\mathrm{S}}^{n} \alpha\right)$ tree and corresponding QCD and EW one-loop Feynman diagrams that contribute to the various parton-level processes in $p p \rightarrow$ $W+1,2,3$ jets. This gives an impression of the complexity of the calculation and its dependence on the jet multiplicity. We observe that the number of one-loop EW diagrams is from $30 \%$ to 3 times higher as compared to the case of one-loop QCD. Moreover, as discussed below, the NLO EW corrections to four-quark processes require both one-loop EW and one-loop QCD diagrams. The number of one-loop EW diagrams increases by about one order of magnitude for each extra jet, similarly as in the one-loop QCD case, and for $W+3 j$ production it ranges from about 1000 to 2600 per partonic subprocess. 


\begin{tabular}{|lllll|}
\hline Channel & QCD trees & EW trees & QCD 1-loop & EW 1-loop \\
\hline$u_{i} \bar{d}_{i} \rightarrow W^{+} g$ & 2 & - & 11 & 32 \\
$u_{i} \bar{d}_{i} \rightarrow W^{+} q \bar{q}$ & $2(4)$ & $7(14)$ & $33(66)$ & $105(210)$ \\
$u_{i} \bar{d}_{i} \rightarrow W^{+} g g$ & 8 & - & 150 & 266 \\
$u_{i} \bar{d}_{i} \rightarrow W^{+} q \bar{q} g$ & $12(24)$ & $33(66)$ & $352(704)$ & $1042(2084)$ \\
$u_{i} \bar{d}_{i} \rightarrow W^{+} g g g$ & 54 & - & 2043 & 2616 \\
\hline
\end{tabular}

Table 1. Number of tree and one-loop Feynman diagrams in the various $p p \rightarrow W^{+}+n$-jet partonic subprocesses: QCD trees of $\mathcal{O}\left(g_{\mathrm{S}}^{n} e\right)$, EW trees of $\mathcal{O}\left(g_{\mathrm{S}}^{n-2} e^{3}\right)$, 1-loop QCD diagrams of $\mathcal{O}\left(g_{\mathrm{S}}^{n+2} e\right)$, and 1-loop EW diagrams of $\mathcal{O}\left(g_{\mathrm{S}}^{n} e^{3}\right)$. Numbers in parenthesis refer to the case of four-quark processes with same flavour, $q=u_{i}$ or $q=d_{i}$. In the OpenLoops framework individual contributions associated with the three independent colour structures of four-gluon vertices count as separate diagrams.

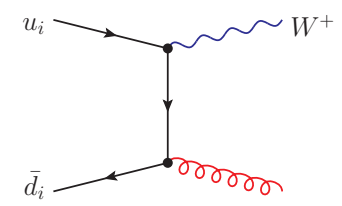

(a)

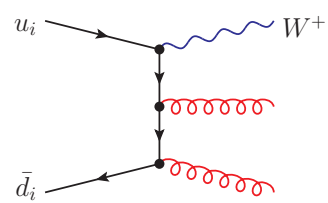

(b)

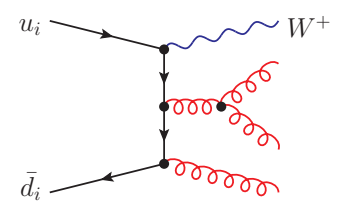

(c)

Figure 5. Representative tree diagrams for $u_{i} \bar{d}_{i} \rightarrow W^{+}+n$-gluon matrix elements at $\mathcal{O}\left(g_{\mathrm{S}}^{n} e\right)$.

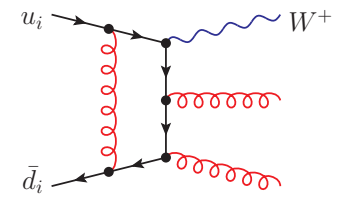

(a)

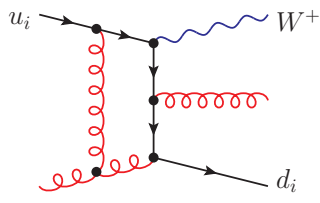

(b)

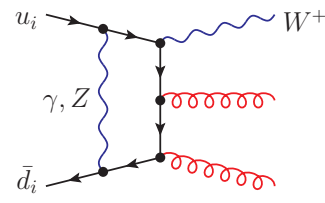

(c)

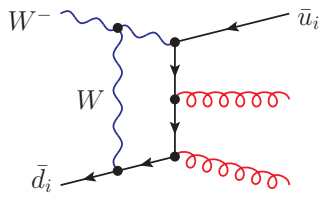

(d)

Figure 6. Representative one-loop diagrams for $u_{i} \bar{d}_{i} \rightarrow W^{+} g g$ matrix elements at $\mathcal{O}\left(g_{\mathrm{S}}^{4} e\right)(6 \mathrm{a}-6 \mathrm{~b})$ and $\mathcal{O}\left(g_{\mathrm{S}}^{2} e^{3}\right)(6 \mathrm{c}-6 \mathrm{~d})$.

\subsection{Two-quark contributions to $p p \rightarrow W^{+}+n$ jets}

Due to the presence of a single quark pair, the $u_{i} \bar{d}_{i} \rightarrow W^{+}+n$-gluon channels feature a rather simple structure from the viewpoint of EW interactions: the $W$ boson is necessarily coupled to the $u_{i} \bar{d}_{i}$ quark line, while gluons can be produced only through strong interactions. Representative tree diagrams for processes with $n=1,2,3$ gluons are depicted in figure 5. For each of these two-quark channels, tree-level amplitudes are characterised by a unique order, $g_{\mathrm{S}}^{n} e$. Thus at NLO each one-loop Feynman diagram can be uniquely assigned either to the QCD or to the EW corrections, depending on its order in $g_{\mathrm{S}}$ and $e$.

Examples of one-loop and real-emission diagrams that contribute to the NLO $\mathrm{QCD}+\mathrm{EW}$ corrections to $u_{i} \bar{d}_{i} \rightarrow W^{+} g g$ are displayed in figures 6 and 7 , respectively. Corresponding diagrams for $p p \rightarrow W^{+}+1 j$ and $p p \rightarrow W^{+}+3 j$ are obtained by removing or adding an external gluon, or for the case of $W^{+}+3 j$ by replacing an external gluon with 


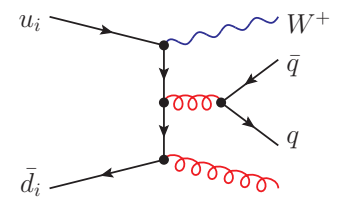

(a)

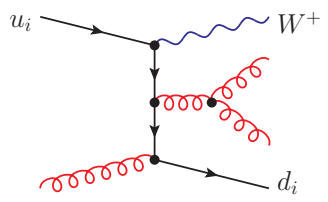

(b)

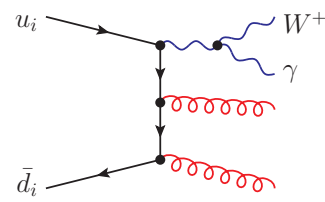

(c)

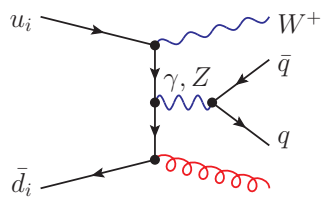

(d)

Figure 7. Representative diagrams for the real corrections to $u_{i} \bar{d}_{i} \rightarrow W^{+} g g$ : contributions to the $\mathcal{O}\left(g_{\mathrm{S}}^{3} e\right)$ QCD emission amplitudes $(7 \mathrm{a}-7 \mathrm{~b})$, the $\mathcal{O}\left(g_{\mathrm{S}}^{2} e^{2}\right)$ QED emission amplitudes $(7 \mathrm{c})$ and the $\mathcal{O}\left(g_{\mathrm{S}} e^{3}\right) q \bar{q}$ emission amplitudes $(7 \mathrm{~d})$.

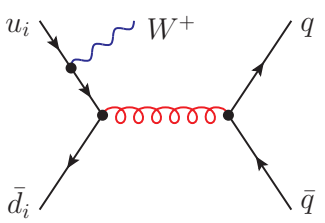

(a)

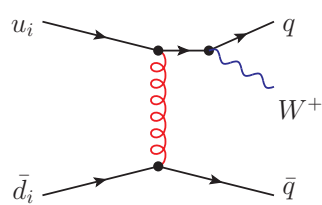

(b)

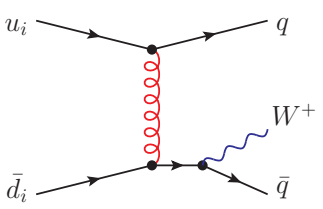

(c)

Figure 8. Representative tree diagrams for $u_{i} \bar{d}_{i} \rightarrow W^{+} q \bar{q}$ matrix elements at $\mathcal{O}\left(g_{\mathrm{S}}^{2} e\right)$. While $s$-channel gluon exchange (8a) contributes to any flavour configuration with $q \in\{u, d, s, c, b\}, t$ channel topologies of type 8b and 8c contribute only when $q=d_{i}$ and $q=u_{i}$, respectively.

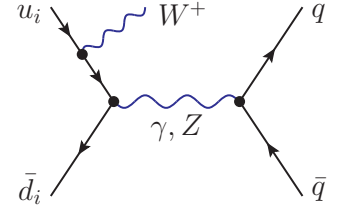

(a)

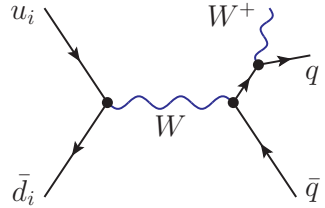

(b)

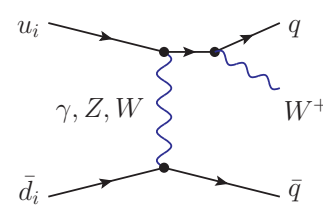

(c)

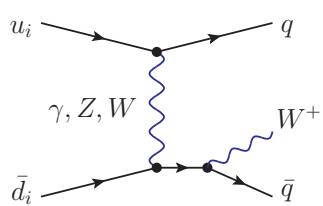

(d)

Figure 9. Representative tree diagrams for $u_{i} \bar{d}_{i} \rightarrow W^{+} q \bar{q}$ matrix elements at $\mathcal{O}\left(e^{3}\right)$. While $s$-channel exchange of EW bosons $(9 \mathrm{a}-9 \mathrm{~b})$ contributes to any flavour configuration with $q \in$ $\{u, d, s, c, b\}$, processes with $q=d_{i}\left(q=u_{i}\right)$ receive also contributions from topologies of type 9c with $t$-channel exchange of neutral (charged) EW bosons and topologies of type $9 \mathrm{~d}$ with $t$-channel exchange of charged (neutral) EW bosons.

a $q \bar{q}$ pair. The $\mathcal{O}\left(\alpha_{S}^{3} \alpha\right)$ NLO QCD corrections to $u_{i} \bar{d}_{i} \rightarrow W^{+} g g$ receive contributions from the interference of $\mathcal{O}\left(g_{\mathrm{S}}^{2} e\right)$ trees $(5 \mathrm{~b})$ with $\mathcal{O}\left(g_{\mathrm{S}}^{4} e\right)$ loop diagrams (6a-6b), and from squared $\mathcal{O}\left(g_{\mathrm{S}}^{3} e\right)$ QCD emission amplitudes $(7 \mathrm{a}-7 \mathrm{~b})$, while the $\mathcal{O}\left(\alpha_{\mathrm{S}}^{2} \alpha^{2}\right)$ NLO EW corrections to the same process receive contributions from the interference of $\mathcal{O}\left(g_{\mathrm{S}}^{2} e\right)$ trees $(5 \mathrm{~b})$ with $\mathcal{O}\left(g_{\mathrm{S}}^{2} e^{3}\right)$ loop diagrams (6c-6d), from squared $\mathcal{O}\left(g_{\mathrm{S}}^{2} e^{2}\right)$ QED emission amplitudes (7c) and from the interference of $\mathcal{O}\left(g_{\mathrm{S}}^{3} e\right)$ (figure $7 \mathrm{a}$ ) and $\mathcal{O}\left(g_{\mathrm{S}} e^{3}\right)$ (figure $7 \mathrm{~d}$ ) $q \bar{q}$ emission diagrams.

\subsection{Four-quark contributions to $p p \rightarrow W^{+}+n$ jets}

The production of $W$ bosons in association with two and three jets involves also the fourquark processes (4.2) and (4.4), respectively. In this case, the possibility to couple the two quark lines either through gluons or EW bosons gives rise to a nontrivial interplay between QCD and EW interactions already at tree-level. In the following we will discuss such effects 


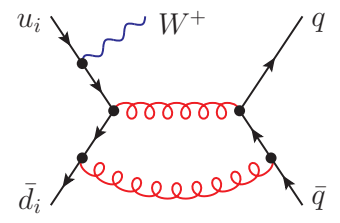

(a)

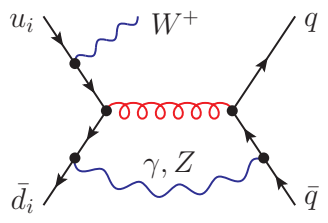

(b)

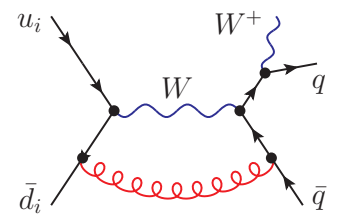

(c)

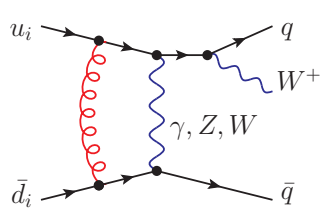

(d)

Figure 10. Representative one-loop diagrams for $u_{i} \bar{d}_{i} \rightarrow W^{+} q \bar{q}$ matrix elements at $\mathcal{O}\left(g_{\mathrm{S}}^{4} e\right)$ (10a) and $\mathcal{O}\left(g_{\mathrm{S}}^{2} e^{3}\right)(10 \mathrm{~b}-10 \mathrm{~d})$. The $s$-channel topologies $(10 \mathrm{a}-10 \mathrm{c})$ contribute to any process with $q \in$ $\{u, d, s, c, b\}$. For $q=b$, diagrams of type 10c involve resonant top-quark propagators. Diagrams of type 10d, with $t$-channel exchange of neutral (charged) EW bosons contribute only to processes with $q=d_{i}\left(q=u_{i}\right)$.

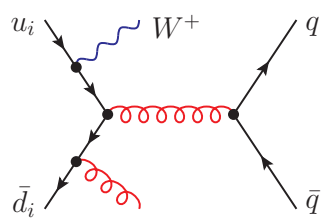

(a)

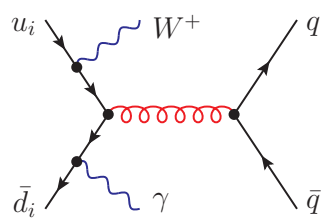

(b)

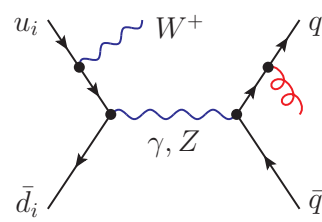

(c)

Figure 11. Representative diagrams for the real corrections to $u_{i} \bar{d}_{i} \rightarrow W^{+} q \bar{q}$ : contributions to the $\mathcal{O}\left(g_{\mathrm{S}}^{3} e\right)$ QCD emission amplitudes (11a), $\mathcal{O}\left(g_{\mathrm{S}}^{2} e^{2}\right)$ QED emission amplitudes (11b), and $\mathcal{O}\left(g_{\mathrm{S}} e^{3}\right)$ QCD emission amplitudes (11c).

in the context of the NLO QCD+EW corrections to $u_{i} \bar{d}_{i} \rightarrow W^{+} q \bar{q}$. Representative LO and NLO Feynman diagrams for this process are displayed in figures 8-11, while corresponding diagrams for $u_{i} \bar{d}_{i} \rightarrow W^{+} q \bar{q} g$ are easily obtained by adding an external gluon or, in the case of NLO emissions, by converting a gluon into an additional $q \bar{q}$ pair.

At tree level, $u_{i} \bar{d}_{i} \rightarrow W^{+} q \bar{q}$ scattering amplitudes receive QCD contributions of $\mathcal{O}\left(g_{\mathrm{S}}^{2} e\right)$ (figure 8) as well as EW contributions of $\mathcal{O}\left(e^{3}\right)$ (figure 9). Squared QCD amplitudes, mixed EW-QCD amplitudes, and squared EW amplitudes, result in cross section contributions of $\mathcal{O}\left(\alpha_{\mathrm{S}}^{2} \alpha\right), \mathcal{O}\left(\alpha_{\mathrm{S}} \alpha^{2}\right)$, and $\mathcal{O}\left(\alpha^{3}\right)$, respectively. In this paper we mainly focus on the leading QCD contributions of $\mathcal{O}\left(\alpha_{\mathrm{S}}^{2} \alpha\right)$ and related NLO QCD+EW corrections. Nevertheless, in section 6 we will discuss also the impact of mixed Born contributions of $\mathcal{O}\left(\alpha_{\mathrm{S}} \alpha^{2}\right)$ arising in the four- quark channel. In general, all Born contributions are relevant, and their simulation is in principle straightforward. However, the EW contributions of type 9a-9d involve various unstable particles that can give rise to resonances: besides topologies where an external quark-antiquark pair is coupled to a $Z$ or $W$ boson propagator (figures 9a-9d), in the case of $u_{i} \bar{d}_{i} \rightarrow W^{+} b \bar{b}$ and crossing related channels also top-quark propagators coupled to external $W b$ pairs can occur (figure 9b). As a consequence, pure EW $\mathcal{O}\left(\alpha^{3}\right)$ contributions to $W+2 j$ production involve $Z, W$, and top resonances that need to be regularised in a consistent way by means of the relevant widths, $\Gamma_{Z, W, t}$. These resonant contributions correspond to $W Z, W W$, and $t j$ production with $Z \rightarrow j j, W \rightarrow j j$ and $t \rightarrow W b$ decays, respectively. However, $W+2 j$ production at $\mathcal{O}\left(\alpha^{3}\right)$ contains also nonresonant contributions to the same final states, and interferences between resonant and 
non-resonant amplitudes. Therefore, contributions of $\mathcal{O}\left(\alpha^{3}\right)$ can not unambiguously be assigned to either $W W, W Z, t j$ or to $W+2 j$ production. As far as the EW-QCD interference contributions of $\mathcal{O}\left(\alpha_{\mathrm{S}} \alpha^{2}\right)$ are concerned, due to the interference with QCD diagrams, ${ }^{9}$ the $Z, W$, and top propagators in the EW amplitudes do not lead to any Breit-Wigner peak in $p p \rightarrow W+j j$ distributions.

Examples of one-loop and real emission diagrams that contribute to $u_{i} \bar{d}_{i} \rightarrow W^{+} q \bar{q}$ at NLO QCD+EW are presented in figures 10 and 11. The $\mathcal{O}\left(\alpha_{\mathrm{S}}^{3} \alpha\right)$ NLO QCD corrections receive contributions from the interference of $\mathcal{O}\left(g_{\mathrm{S}}^{2} e\right)$ trees $(8 \mathrm{a}-8 \mathrm{c})$ with $\mathcal{O}\left(g_{\mathrm{S}}^{4} e\right)$ loop diagrams (10a), and from the squared $\mathcal{O}\left(g_{\mathrm{S}}^{3} e\right)$ QCD emission amplitudes (11a). The $\mathcal{O}\left(\alpha_{\mathrm{S}}^{2} \alpha^{2}\right)$ NLO EW corrections involve contributions that arise from the interference of QCD trees of $\mathcal{O}\left(g_{\mathrm{S}}^{2} e\right)$ (figures 8a-8c) with loop diagrams of $\mathcal{O}\left(g_{\mathrm{S}}^{2} e^{3}\right)$ (figures $10 \mathrm{~b}-10 \mathrm{~d}$ ), and from the squared QED emission amplitudes of $\mathcal{O}\left(g_{\mathrm{S}}^{2} e^{2}\right)$ (figure 11b). In addition, the NLO EW corrections involve also interferences of EW trees of $\mathcal{O}\left(e^{3}\right)$ (figures $9 \mathrm{a}-9 \mathrm{~d}$ ) with $\mathcal{O}\left(g_{\mathrm{S}}^{4} e\right)$ loop diagrams (10a), as well as interferences between QCD real emission amplitudes of $\mathcal{O}\left(g_{\mathrm{S}}^{3} e\right)$ (figure 11a) and $\mathcal{O}\left(g_{\mathrm{S}} e^{3}\right)$ (figure 11c). Similarly as in the Born case, EW-QCD interference terms at NLO EW order $\alpha_{\mathrm{S}}^{2} \alpha^{2}$ do not give rise to Breit-Wigner resonances in $W+2 j$ production. The same holds for EW-QCD interference terms of order $\alpha_{\mathrm{S}}^{3} \alpha^{2}$ in $p p \rightarrow W+3 j$.

\subsection{Photon-induced processes}

At tree level, if one treats the photon density as a quantity of $\mathcal{O}(1)$ as discussed in section 2.3, $W+$ multijet production receives Born contributions from $\gamma p \rightarrow W+n$ jets at $\mathcal{O}\left(\alpha_{\mathrm{S}}^{n-1} \alpha^{2}\right)$, i.e. at the same order as EW-QCD interference terms, as well as $\gamma \gamma \rightarrow W+n$ jet contributions at $\mathcal{O}\left(\alpha_{\mathrm{S}}^{n-2} \alpha^{3}\right)$, which is the order of pure EW Born terms. Photon-photon channels start contributing at $n=2$. More explicitly, $W^{+} j$ production receives $\gamma p \rightarrow W^{+} j$ contributions of $\mathcal{O}\left(\alpha^{2}\right)$ from the partonic process

$$
\gamma u_{i} \rightarrow W^{+} d_{i}
$$

and crossing-related channels. Hadro-production of $W+2$ jets involves the following singlephoton induced processes of $\mathcal{O}\left(\alpha_{\mathrm{S}} \alpha^{2}\right)$ and $\gamma \gamma$-induced processes of $\mathcal{O}\left(\alpha^{3}\right)$,

$$
\begin{aligned}
\gamma u_{i} & \rightarrow W^{+} d_{i} g, \\
\gamma \gamma & \rightarrow W^{+} d_{i} \bar{u}_{i},
\end{aligned}
$$

while $W+3 j$ production involves the following $\mathcal{O}\left(\alpha_{\mathrm{S}}^{2} \alpha^{2}\right)$ single-photon induced and $\mathcal{O}\left(\alpha_{\mathrm{S}} \alpha^{3}\right)$ $\gamma \gamma$-induced channels,

$$
\begin{aligned}
\gamma u_{i} & \rightarrow W^{+} d_{i} g g, \\
\gamma \gamma & \rightarrow W^{+} d_{i} \bar{u}_{i} g,
\end{aligned}
$$

\footnotetext{
${ }^{9}$ For $p p \rightarrow W+2 j$, such Born interferences are possible only in presence of the colour flow associated with $t$-channel contributions of type $8 \mathrm{~b}-8 \mathrm{c}$ and $9 \mathrm{c}-9 \mathrm{~d}$, i.e. only for same-flavour quark combinations with $q=u_{i}$ or $q=d_{i}$. If $u_{i} \bar{d}_{i} \rightarrow W^{+} q \bar{q}$ amplitudes are dressed with an extra (virtual or real) gluon, then EW-QCD interferences contribute to all flavour combinations $q=u, d, s, c, b$.
} 
together with the following channel contributing at $\mathcal{O}\left(\alpha_{\mathrm{S}}^{2} \alpha^{2}\right), \mathcal{O}\left(\alpha_{\mathrm{S}} \alpha^{3}\right)$ and $\mathcal{O}\left(\alpha^{4}\right)$,

$$
\gamma u_{i} \rightarrow W^{+} d_{i} q \bar{q}
$$

All $\gamma p$ - and $\gamma \gamma$-induced processes enter at a different (lower) order in $\alpha_{\mathrm{S}}$ as compared to the NLO EW corrections of $\mathcal{O}\left(\alpha_{\mathrm{S}}^{n} \alpha^{2}\right)$ presented in this paper. Photon-induced contributions are thus irrelevant for the cancellation of collinear initial-state singularities at $\mathcal{O}\left(\alpha_{\mathrm{S}}^{n} \alpha^{2}\right)$, and can be handled as separate processes. From the formal power-counting perspective, leading $\gamma p$-induced processes are actually more important than NLO EW corrections, but in most of the phase space they are strongly suppressed by the small photon PDF. However, as we will see in section 6 , in the very high-energy tails of distributions photon-induced processes can have a sizable impact on $W+$ multijet production. As is well known, this is due to the relative enhancement of the photon density at large $x$. At the same time, the poor knowledge of the photon PDF in this kinematic region represents a large source of theoretical uncertainty [68].

\subsection{Technical aspects of the on-shell approximation}

In this paper we consider $W+$ multijet production with stable on-shell $W$ bosons, and the inclusion of $W \rightarrow \ell \nu$ decays will be addressed in a subsequent publication. Implementing $W$ boson decays at NLO EW does not represent a dramatic source of extra complexity as long as $W$ bosons are kept on-shell, such that $W$ +multijet production and $W$ decays can be factorised using the narrow-width approximation (NWA). In contrast, a full description of $p p \rightarrow \ell \nu+n$ jets, including off-shell contributions at NLO EW, would be at least one order of magnitude more CPU expensive. This is simply due to the fact that, if the $W$ boson is replaced by a $\ell \nu$ pair, the number of external particles that can enter EW loops increases by one. ${ }^{10}$ Keeping the external $W$ boson on-shell - either as stable particle or as decaying particle in NWA - is thus essential in order to be able to push $W+$ multijet NLO EW calculations up to the highest possible jet multiplicity while keeping the complexity at a manageable level.

Unfortunately, the simplifications that arise from the on-shell (or narrow-width) approximation are accompanied by some technical complications at NLO EW. The key problem is that the on-shell treatment of external $W$ bosons implies that the $W$ boson width is set to zero, while EW corrections give rise to internal $W$ propagators that can produce physical resonances, which requires a non-zero width. In presence of physical resonances, it is clear that all $W$ bosons must be consistently handled as unstable particles with nonzero width, and in order to preserve gauge invariance the complex-mass scheme [99] has to be used, which means that the on-shell description of $W+$ multijet production has to be abandoned (or improved in a nontrivial way that preserves gauge invariance). However, as discussed in section 4.3, internal $W, Z$, and top propagators that enter the EW corrections to $p p \rightarrow W+n$ jets cannot give rise to Breit-Wigner resonances at $\mathcal{O}\left(\alpha_{\mathrm{S}}^{n} \alpha^{2}\right)$. At this perturbative order, resonant $Z, W$, and $t$ propagators appear in the EW Born amplitudes of

\footnotetext{
${ }^{10}$ Note that off-shell $W \rightarrow \ell \nu$ decays are trivial at NLO QCD as they do not increase the number of external lines that enter QCD loops.
} 


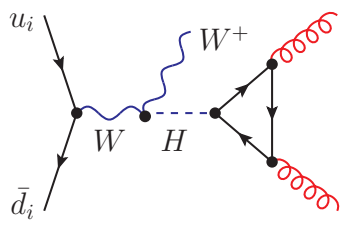

(a)

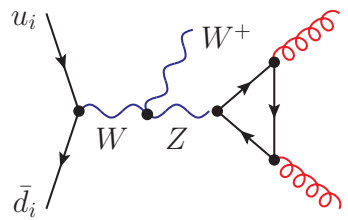

(b)

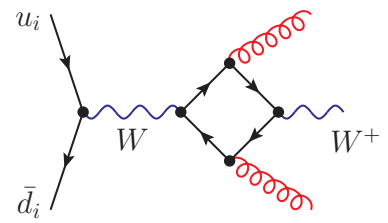

(c)

Figure 12. Examples of 1-loop $u_{i} \bar{d}_{i} \rightarrow W^{+} g g$ matrix elements at $\mathcal{O}\left(g_{\mathrm{S}}^{2} e^{3}\right)$ that involve potentially resonant Higgs-boson (12a), $Z$-boson (12b) and $W$-boson (12c) propagators, where the last example diagram can only become resonant in the $g g \rightarrow W^{+} \bar{u}_{i} d_{i}$ crossing.

$\mathcal{O}\left(g_{\mathrm{S}}^{n-2} e^{3}\right)$ (see $\left.9 \mathrm{a}-9 \mathrm{c}\right)$, in the EW virtual amplitudes of $\mathcal{O}\left(g_{\mathrm{S}}^{n} e^{3}\right)$ (see $10 \mathrm{~b}-10 \mathrm{~d}$ ), and in the QCD emission amplitudes of $\mathcal{O}\left(g_{\mathrm{S}}^{n-1} e^{3}\right)$ (see 11c), but they contribute to the physical cross section only through interference with non-resonant QCD amplitudes. As illustrated in figure 12, also two-quark processes involve EW 1-loop topologies with potentially resonant particles, including Higgs bosons. In any case, as a result of the interference with QCD amplitudes, none of these contributions can give rise to a physical resonance.

Since $p p \rightarrow W+n$ jets at $\mathcal{O}\left(\alpha_{\mathrm{S}}^{n} \alpha^{2}\right)$ is free from Breit-Wigner resonances, in principle the $W$ width can be set to zero in all scattering amplitudes, consistently with the on-shell treatment of external $W$ bosons. However, the interference of resonant and non-resonant contributions gives rise to spikes that can disturb the numerical stability of the phasespace integration in the vicinity of the "pseudo-resonance". An optimal treatment of these regions can be achieved by introducing an ad-hoc technical width $\Gamma_{\text {reg }}$ in the potentially resonant propagators, in such a way that the pseudo-resonant contributions behave as

$$
\lim _{Q^{2} \rightarrow M^{2}} \frac{\mathrm{d} \sigma}{\mathrm{d} Q^{2}} \propto \frac{Q^{2}-M^{2}}{\left(Q^{2}-M^{2}\right)^{2}+\Gamma_{\mathrm{reg}}^{2} M^{2}} .
$$

The idea is that the $1 / \Gamma_{\text {reg }}^{2}$ enhancement at $Q^{2} \sim M^{2}$ cancels upon integration over $Q^{2}$, and the overall dependence on the technical regulator should be $\mathcal{O}\left(\Gamma_{\text {reg }} / M\right)$ suppressed, while all contributions should formally behave smoothly when $\Gamma_{\text {reg }} \rightarrow 0$. If these conditions are fulfilled, then the calculation should consistently converge towards the correct on-shell limit, and using a sufficiently small value for $\Gamma_{\text {reg }}$ should guarantee a negligible numerical impact of $\mathcal{O}\left(\Gamma_{\text {reg }} / M\right)$ effects and related violations of gauge invariance.

In this context, due to the presence of IR singularities that arise from (virtual and real) soft photons coupled to external $W$ bosons, the smooth convergence of the $\Gamma_{\text {reg }} \rightarrow 0$ limit represents a nontrivial requirement. In fact, a naive introduction of $\Gamma_{\text {reg }}>0$ in all $W$ propagators would turn such soft-photon singularities into $\ln \left(\Gamma_{\text {reg }}\right)$ terms that do not converge towards the correct $1 /(D-4)$ poles when $\Gamma_{\text {reg }} \rightarrow 0$. Fortunately, all diagrams that involve real or virtual photons are free from potential resonances. Therefore, in order to guarantee a smooth $\Gamma_{\text {reg }} \rightarrow 0$ behaviour, one can simply restrict the $\Gamma_{\text {reg }}>0$ regulator to those diagrams that are free from photons, and evaluate all photonic corrections at zero width. More precisely, we will adopt the following approach, which is applicable at $\mathcal{O}\left(\alpha_{\mathrm{S}}^{n} \alpha^{2}\right)$ for the case of stable $W$ bosons as well as for decaying $W$ bosons in NWA: 
- the physical width of all unstable particles $(W, Z, t, H)$ is never included in the corresponding propagators, and the corresponding masses, as well as the related mixing angles and Higgs couplings, are treated as real parameters, i.e. the complex mass scheme is not used;

- external $W$ bosons are kept on their mass shell, $p_{W}^{2}=M_{W}^{2}$;

- in diagrams that do not involve photons, possible $W, Z, H$, and top-quark propagators are regularised as $1 /\left(Q^{2}-M^{2}+i \Gamma_{\text {reg }} M\right)$ with a small technical width $\Gamma_{\text {reg. }}$.

The dependence of physical observables on the value of $\Gamma_{\text {reg }}$ must be regarded as a small uncertainty associated with a gauge-dependent $\mathcal{O}\left(\Gamma_{\text {reg }}\right)$ deformation around the exact gaugeinvariant limit $\Gamma_{\text {reg }} \rightarrow 0$ limit. In this respect, it should be stressed that, thanks to the smooth convergence of the $\Gamma_{\text {reg }} \rightarrow 0$ limit, these violations of gauge invariance are controllable, in the sense that they can be quantified and systematically reduced by chosing an appropriate $\Gamma_{\text {reg value. }}{ }^{11}$

Results presented in section 6 have been obtained using $\Gamma_{\text {reg }}=1 \mathrm{GeV}$, which turns out to guarantee good numerical stability and negligible $\Gamma_{\text {reg }}$ dependence. More precisely, we have checked that for all integrated and differential results presented in section 6 the shift resulting from variations of $\Gamma_{\text {reg }}$ between 0.1 and $1 \mathrm{GeV}$ is well below one percent.

\section{Setup of the simulation}

As input parameters to simulate $W+$ multijet production at NLO QCD+EW we use the gauge-boson, Higgs-boson, and top-quark masses

$$
M_{\mathrm{Z}}=91.1876 \mathrm{GeV}, \quad M_{\mathrm{W}}=80.385 \mathrm{GeV}, \quad M_{\mathrm{H}}=126 \mathrm{GeV}, \quad m_{\mathrm{t}}=173.2 \mathrm{GeV} .(5.1)
$$

The corresponding Lagrangian parameters are kept strictly real since we treat all heavy particles as stable. The electroweak couplings are derived from the gauge-boson masses and the Fermi constant, $G_{\mu}=1.16637 \times 10^{-5} \mathrm{GeV}^{-2}$, in the $G_{\mu}$-scheme (3.6). The CKM matrix is assumed to be diagonal, while colour effects and related interferences are included throughout, without applying any large- $N_{c}$ expansion.

For the calculation of hadron-level cross sections we employ the NNPDF2.3 QED parton distributions [68], which include NLO QCD and LO QED effects, and we use the PDF set corresponding to $\alpha_{\mathrm{S}}\left(M_{\mathrm{Z}}\right)=0.118 .{ }^{12}$ Matrix elements are evaluated using the running strong coupling supported by the PDFs and, consistently with the variable flavournumber scheme implemented in the NNPDFs, at the top threshold we switch from five to six active quark flavours in the renormalisation of $\alpha_{\mathrm{S}}$. All light quarks, including bottom quarks, are treated as massless particles, and top-quark loops are included throughout in the calculation. The NLO PDF set is used for LO as well as for NLO QCD and NLO EW predictions. Using the same PDFs for LO and NLO predictions exposes matrix-element

\footnotetext{
${ }^{11}$ This is completely different with respect to violations of gauge invariance in process that involve physical resonances.

${ }^{12}$ To be precise we use the NNPDF23_nlo_as_0118_qed set interfaced through the LHAPDF library 5.9.1.
} 
correction effects in a more transparent way. In particular, it guarantees that NLO EW $K$ factors remain free from QCD effects related to the difference between LO and NLO PDFs.

The renormalisation scale $\mu_{R}$ and factorisation scale $\mu_{F}$ are set to

$$
\mu_{\mathrm{R}, \mathrm{F}}=\xi_{\mathrm{R}, \mathrm{F}} \mu_{0} \quad \text { with } \quad \mu_{0}=\hat{H}_{\mathrm{T}} / 2,
$$

where $\hat{H}_{\mathrm{T}}$ is the scalar sum of the transverse energy of all partonic final-state particles,

$$
\hat{H}_{\mathrm{T}}=\sum_{\text {partons }} E_{\mathrm{T}}=\sum_{i} E_{\mathrm{T}, j_{i}}+E_{\mathrm{T}, \gamma}+\sqrt{p_{\mathrm{T}, \mathrm{W}}^{2}+M_{W}^{2}} .
$$

Our default scale choice corresponds to $\xi_{\mathrm{R}}=\xi_{\mathrm{F}}=1$, and theoretical uncertainties are assessed by applying the scale variations $\left(\xi_{\mathrm{R}}, \xi_{\mathrm{F}}\right)=(2,2),(2,1),(1,2),(1,1),(1,0.5)$, $(0.5,1),(0.5,0.5)$. As shown in $[20-25]$ the scale choice $(5.2)$ guarantees a good perturbative convergence for $W+$ multijet production over a wide range of observables and energy scales.

For the definition of jets we employ the anti- $k_{\mathrm{T}}$ algorithm [110] with $R=0.4$. More precisely, in order to guarantee IR safeness in presence of NLO QCD and EW corrections, we adopt the democratic clustering approach introduced in section 2.4, treating QCD jets and photons as separate physics objects. To this end we impose an upper bound $z_{\mathrm{thr}}=0.5$ to the photon energy fraction inside jets, and the recombination of collinear (anti)quarkphoton pairs is applied within a cone of radius $R_{\gamma q}^{\mathrm{rec}}=0.1$.

\section{NLO QCD+EW predictions for $W^{+}+1,2,3$ jets at the LHC}

In the following we present a series of NLO QCD+EW simulations for $W^{+}$production in association with one, two, and three jets in proton-proton collisions at $13 \mathrm{TeV}$. Events are categorised according to the number of jets in the transverse-momentum and pseudorapidity region defined by

$$
p_{\mathrm{T}, j}>30 \mathrm{GeV}, \quad\left|\eta_{j}\right|<4.5,
$$

and for each $W+n$-jet sample we present an inclusive analysis, where we do not impose any selection cut apart from requiring the presence of $n$ (or more) jets. In addition, to study the high-energy behaviour of EW corrections, we also consider cross sections and distributions in presence of one of the following cuts:

$$
p_{\mathrm{T}, \mathrm{W}}>1 \mathrm{TeV}, \quad p_{\mathrm{T}, j_{1}}>1 \mathrm{TeV}, \quad \text { or } \quad H_{\mathrm{T}}^{\mathrm{tot}}>2 \mathrm{TeV} .
$$

Here $j_{1}$ denotes the first jet, while the total transverse energy $H_{\mathrm{T}}^{\text {tot }}$ is defined in terms of the jet and $W$-boson transverse momenta ${ }^{13}$ as

$$
H_{\mathrm{T}}^{\mathrm{tot}}=p_{\mathrm{T}, \mathrm{W}}+\sum_{k} p_{\mathrm{T}, j_{k}}
$$

where all jets that satisfy (6.1) are included.

\footnotetext{
${ }^{13}$ Note that at variance with the definition (5.3) of $\hat{H}_{\mathrm{T}}$, here we use transverse momenta and not transverse energies.
} 
Our default NLO results are obtained by combining QCD and EW predictions,

$$
\sigma_{\mathrm{QCD}}^{\mathrm{NLO}}=\sigma^{\mathrm{LO}}+\delta \sigma_{\mathrm{QCD}}^{\mathrm{NLO}}, \quad \sigma_{\mathrm{EW}}^{\mathrm{NLO}}=\sigma^{\mathrm{LO}}+\delta \sigma_{\mathrm{EW}}^{\mathrm{NLO}},
$$

with a standard additive prescription

$$
\sigma_{\mathrm{QCD}+\mathrm{EW}}^{\mathrm{NLO}}=\sigma^{\mathrm{LO}}+\delta \sigma_{\mathrm{QCD}}^{\mathrm{NLO}}+\delta \sigma_{\mathrm{EW}}^{\mathrm{NLO}}
$$

where $\delta \sigma_{\mathrm{QCD}}^{\mathrm{NLO}}$ and $\delta \sigma_{\mathrm{EW}}^{\mathrm{NLO}}$ correspond to $p p \rightarrow W+n$-jet contributions of $\mathcal{O}\left(\alpha_{\mathrm{S}}^{n+1} \alpha\right)$ and $\mathcal{O}\left(\alpha_{\mathrm{S}}^{n} \alpha^{2}\right)$, respectively. As LO contributions, in sections $6.1-6.3$ only the leading-QCD terms of $\mathcal{O}\left(\alpha_{\mathrm{S}}^{n} \alpha\right)$ will be included, while subleading Born contributions and photon-induced terms will be discussed in section 6.4. In order to identify potentially large effects due to the interplay of EW and QCD corrections beyond NLO, we will also consider the following factorised combination of EW and QCD corrections,

$$
\sigma_{\mathrm{QCD} \times \mathrm{EW}}^{\mathrm{NLO}}=\sigma_{\mathrm{QCD}}^{\mathrm{NLO}}\left(1+\frac{\delta \sigma_{\mathrm{EW}}^{\mathrm{NLO}}}{\sigma^{\mathrm{LO}}}\right)=\sigma_{\mathrm{EW}}^{\mathrm{NLO}}\left(1+\frac{\delta \sigma_{\mathrm{QCD}}^{\mathrm{NLO}}}{\sigma^{\mathrm{LO}}}\right) .
$$

If this approach can be justified by a clear separation of scales - such as in situations where QCD corrections are dominated by soft interactions well below the EW scale the factorised formula (6.6) can be regarded as an improved prediction. Otherwise, the difference between (6.5) and (6.6) should be considered as an estimate of unknown higherorder corrections.

In the following sections, we will present $\mathrm{QCD}+\mathrm{EW}$ and $\mathrm{QCD} \times \mathrm{EW}$ NLO corrections relative to $\sigma_{\mathrm{QCD}}^{\mathrm{NLO}}$, which corresponds to the ratios

$$
\begin{aligned}
& \frac{\sigma_{\mathrm{QCD}+\mathrm{EW}}^{\mathrm{NLO}}}{\sigma_{\mathrm{QCD}}^{\mathrm{NLO}}}=\left(1+\frac{\delta \sigma_{\mathrm{EW}}^{\mathrm{NLO}}}{\sigma_{\mathrm{QCD}}^{\mathrm{NLO}}}\right), \\
& \frac{\sigma_{\mathrm{QCD} \times \mathrm{EW}}^{\mathrm{NLO}}}{\sigma_{\mathrm{QCD}}^{\mathrm{NLO}}}=\left(1+\frac{\delta \sigma_{\mathrm{EW}}^{\mathrm{NLO}}}{\sigma^{\mathrm{LO}}}\right) .
\end{aligned}
$$

Note that the $\mathrm{QCD} \times \mathrm{EW}$ ratio (6.8) corresponds to the usual NLO EW correction relative to $\mathrm{LO}$, which is free from NLO QCD effects, while the QCD+EW ratio (6.7) depends on $\sigma_{\mathrm{QCD}}^{\mathrm{NLO}}$. In particular, for observables that receive large NLO QCD corrections, the relative $\mathrm{QCD}+\mathrm{EW}$ correction can be drastically suppressed as compared to the $\mathrm{QCD} \times \mathrm{EW}$ one. This feature is typically encountered in observables that receive huge QCD corrections of real-emission type. In such situations, NLO QCD $+\mathrm{EW}$ predictions for $p p \rightarrow W+n$ jets are dominated by tree-level contributions with one extra jet, and the inclusion of NLO QCD + EW corrections for $p p \rightarrow W+(n+1)$ jets becomes mandatory.

Thanks to the high efficiency of the employed tools, the simulation of $W$ +multijet production at NLO QCD+EW requires a moderate amount of computing resources. The runtime needed to achieve very high statistical accuracy, at the level of $0.1 \%$, in the NLO QCD+EW integrated cross section amounts to about 13, 210 and 6300 CPU hours for $p p \rightarrow W^{+}+1,2,3$ jets, respectively. ${ }^{14}$ In order to obtain $0.1 \%$ statistical accuracy also in

\footnotetext{
${ }^{14}$ The stated runtimes refer to a single core and are estimated from runs on a cluster based on Intel ${ }^{\circledR}$ Xeon ${ }^{\circledR}$ E5-2660 (20MB Cache, 2.20GHz) processors by means of an extrapolation to an overall statistical error of $0.1 \%$ wrt. $\sigma_{\mathrm{QCD}+\mathrm{EW}}^{\mathrm{NLO}}$.
} 


\begin{tabular}{|llllll|}
\hline$W^{+}+1 j$ & inclusive & $\Delta \phi_{j_{1} j_{2}}<3 \pi / 4$ & $H_{\mathrm{T}}^{\text {tot }}>2 \mathrm{TeV}$ & $p_{\mathrm{T}, j_{1}}>1 \mathrm{TeV}$ & $p_{T, W^{+}}>1 \mathrm{TeV}$ \\
\hline$\sigma_{\mathrm{QCD}}^{\mathrm{NLO}}[\mathrm{pb}]$ & $15664_{-5 \%}^{+5 \%}$ & $13429_{-3 \%}^{+2 \%}$ & $0.231_{-20 \%}^{+27 \%}$ & $0.181_{-19 \%}^{+25 \%}$ & $0.050_{-10 \%}^{+10 \%}$ \\
$\sigma_{\mathrm{QCD}+\mathrm{EW}}^{\mathrm{NLO}}[\mathrm{pb}]$ & $15621_{-5 \%}^{+5 \%}$ & $13380_{-3 \%}^{+2 \%}$ & $0.245_{-19 \%}^{+26 \%}$ & $0.195_{-18 \%}^{+25 \%}$ & $0.040_{-8 \%}^{+6 \%}$ \\
$\sigma_{\mathrm{QCD}+\mathrm{EW}}^{\mathrm{NLO}} / \sigma_{\mathrm{QCD}}^{\mathrm{NLO}}$ & $1.00_{-5 \%}^{+5 \%}$ & $1.00_{-3 \%}^{+2 \%}$ & $1.06_{-19 \%}^{+26 \%}$ & $1.07_{-18 \%}^{+25 \%}$ & $0.80_{-8 \%}^{+6 \%}$ \\
$\sigma_{\mathrm{QCD} \times \mathrm{EW}}^{\mathrm{NLO}} / \sigma_{\mathrm{QCD}}^{\mathrm{NLO}}$ & $1.00_{-5 \%}^{+5 \%}$ & $1.00_{-3 \%}^{+2 \%}$ & $1.45_{-18 \%}^{+24 \%}$ & $1.41_{-17 \%}^{+23 \%}$ & $0.70_{-10 \%}^{+9 \%}$ \\
$\sigma^{\mathrm{LO}} / \sigma_{\mathrm{QCD}}^{\mathrm{NLO}}$ & $0.73_{-10 \%}^{+12 \%}$ & $0.86_{-10 \%}^{+12 \%}$ & $0.14_{-18 \%}^{+23 \%}$ & $0.18_{-18 \%}^{+23 \%}$ & $0.65_{-18 \%}^{+23 \%}$ \\
\hline
\end{tabular}

Table 2. Integrated $p p \rightarrow W^{+}+1 j$ cross sections with inclusive cuts (6.1) and in presence of additional cuts. Born cross sections $\left(\sigma^{\mathrm{LO}}\right)$ include only the leading QCD contributions of $\mathcal{O}\left(\alpha_{\mathrm{S}} \alpha\right)$.

the phase-space region with $H_{\mathrm{T}}^{\text {tot }}>2 \mathrm{TeV}$, where the cross section is suppressed by about 4 orders of magnitude, less than a factor 10 of extra CPU time is needed (without using any generation cut). For all processes under consideration, the evaluation of the NLO EW corrections consumes a subleading part of the total CPU budget.

\section{$6.1 W^{+}+1$ jet}

Among the various $W+$ (multi)jet production processes, the inclusive production of a $W$ boson in association with (at least) one jet is the one that features the strongest sensitivity to NLO QCD radiation. This is clearly illustrated by the results shown in figures 13-14 and table 2. In particular, large NLO QCD effects arise in the tails of the inclusive distributions in the $W$-boson and in the jet transverse momenta, shown in the left plot of figure 13. For the $W$-boson $p_{\mathrm{T}}$ distribution NLO QCD corrections exceed $50 \%$, while in the case of the jet- $p_{\mathrm{T}}$ distribution they become as large as $400 \%$ at $1 \mathrm{TeV}$. As is well known, this extreme behaviour is due to the fact that $W$-boson production in association with very hard jets is dominated by $W+$ multijet events where two or more high- $p_{\mathrm{T}}$ jets recoil against each other, while the $W$ boson tends to be rather soft $[7,8,10,35]$. In this kinematic regime, inclusive NLO simulations of $p p \rightarrow W+1 j$ are dominated by tree-level contributions with two jets, which results in large scale uncertainties. The inclusion of NLO corrections for $W+$ multijet production is thus mandatory for a well-behaved theoretical prediction of the inclusive jet- $p_{\mathrm{T}}$ spectrum. Predictions for $p p \rightarrow W+1 j$ at NLO QCD are perturbatively stable only in presence of ad-hoc cuts that separate one-jet configurations form the bulk of the extra jet emission. As shown in the right plot of figure 13, this can be achieved by means of a veto against dijet configurations with azimuthal separation $\Delta \phi_{j j}>3 \pi / 4$. Thanks to this cut, which avoids hard events characterised by a back-two-back dijet system with $\Delta \phi_{j j} \rightarrow \pi$, NLO QCD correction become acceptably small and reasonably stable, even at very large jet $p_{\mathrm{T}}$.

As discussed in the following, the behaviour of NLO EW effects is strictly connected to the one of NLO QCD corrections. The shape of NLO EW corrections to the inclusive $W$ boson $p_{\mathrm{T}}$ distribution (figure 13, left) is consistent with the expected presence of negative Sudakov logarithms that grow as $\ln ^{2}\left(\hat{s} / M_{W}^{2}\right)$. However, in the tail we observe a large gap between $\mathrm{QCD}+\mathrm{EW}$ and $\mathrm{QCD} \times \mathrm{EW}$ predictions, which points to the presence of sizable $\mathrm{EW}$ 

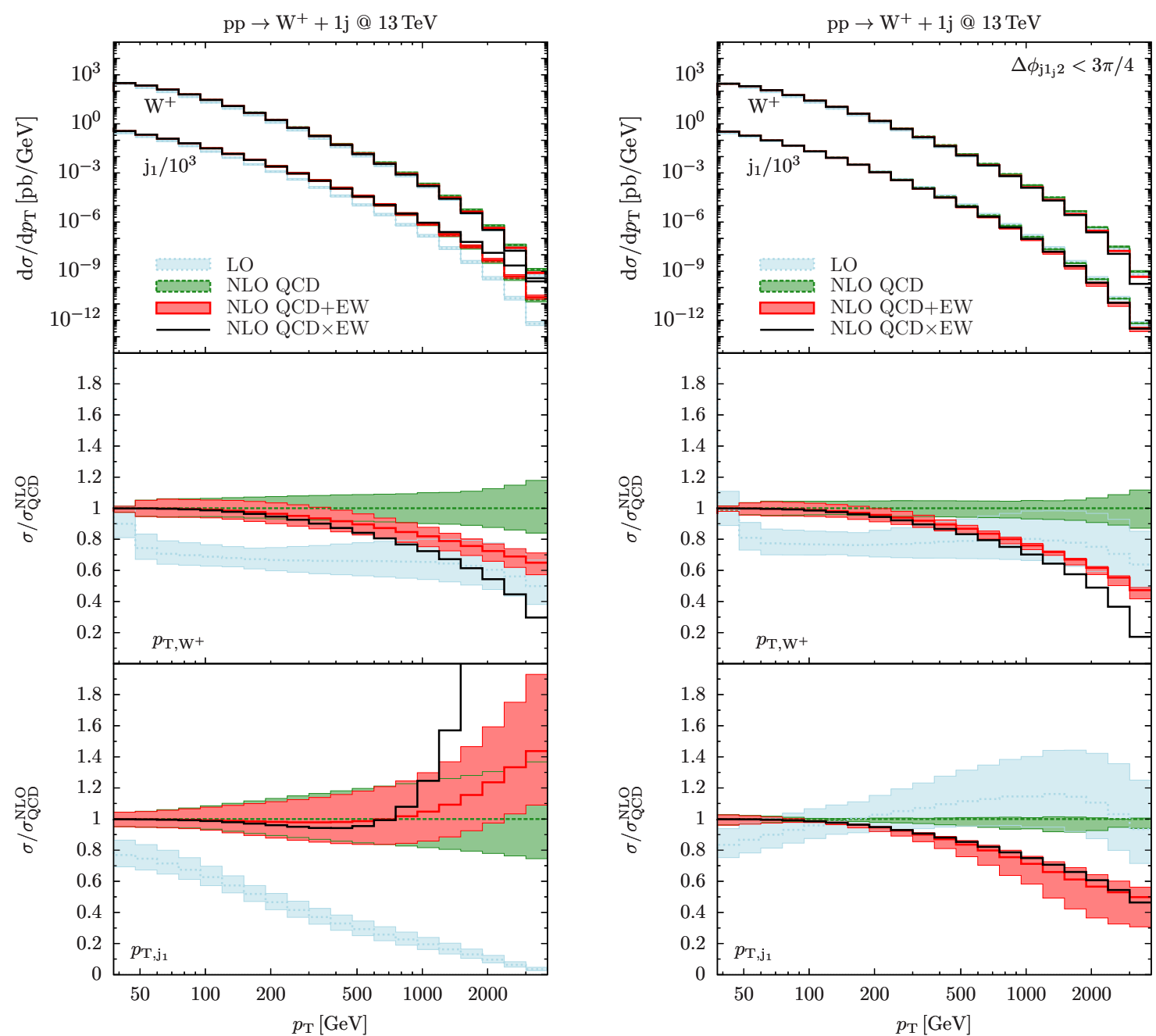

Figure 13. Distributions in the transverse momenta of the $W$ boson and of the first jet for inclusive (left) $W^{+}+1 j$ production and with a cut $\Delta \phi_{j_{1} j_{2}}<3 \pi / 4$ (right). Absolute LO (light blue), NLO QCD (green), NLO QCD+EW (red) and NLO QCD $\times$ EW (black) predictions (upper panel) and relative corrections with respect to NLO QCD (lower panels). The bands correspond to scale variations, and in the case of ratios only the numerator is varied. The distribution in $p_{\mathrm{T}, j_{1}}$ is rescaled by a factor $10^{-3}$.

higher-order effects that are not captured by the NLO QCD+EW approximation. This is clearly due to the fact that the NLO QCD cross section involves large radiative contributions that are effectively described at LO EW accuracy only. In any case, it is clear that NLO EW effects are large. Noteworthy, already for $p_{\mathrm{T}, W} \gtrsim 300 \mathrm{GeV}$ they become larger than the NLO QCD uncertainties. For the inclusive jet- $p_{\mathrm{T}}$ distribution, due to the huge impact of QCD radiation, NLO EW corrections behave in a pathological way. The expected Sudakov suppression is completely absent, and above $1 \mathrm{TeV}$ one observes a strong enhancement. This can be attributed to $\mathcal{O}\left(\alpha_{\mathrm{S}} \alpha^{2}\right)$ mixed EW-QCD contributions to hard-dijet plus soft- $W$ events [35], which result from the interference between diagrams of type $8 \mathrm{a}-8 \mathrm{c}$ and $9 \mathrm{a}-9 \mathrm{~d}$. The increase at large $p_{\mathrm{T}}$ can be understood as a PDF effect at large Bjoerken $x$. 

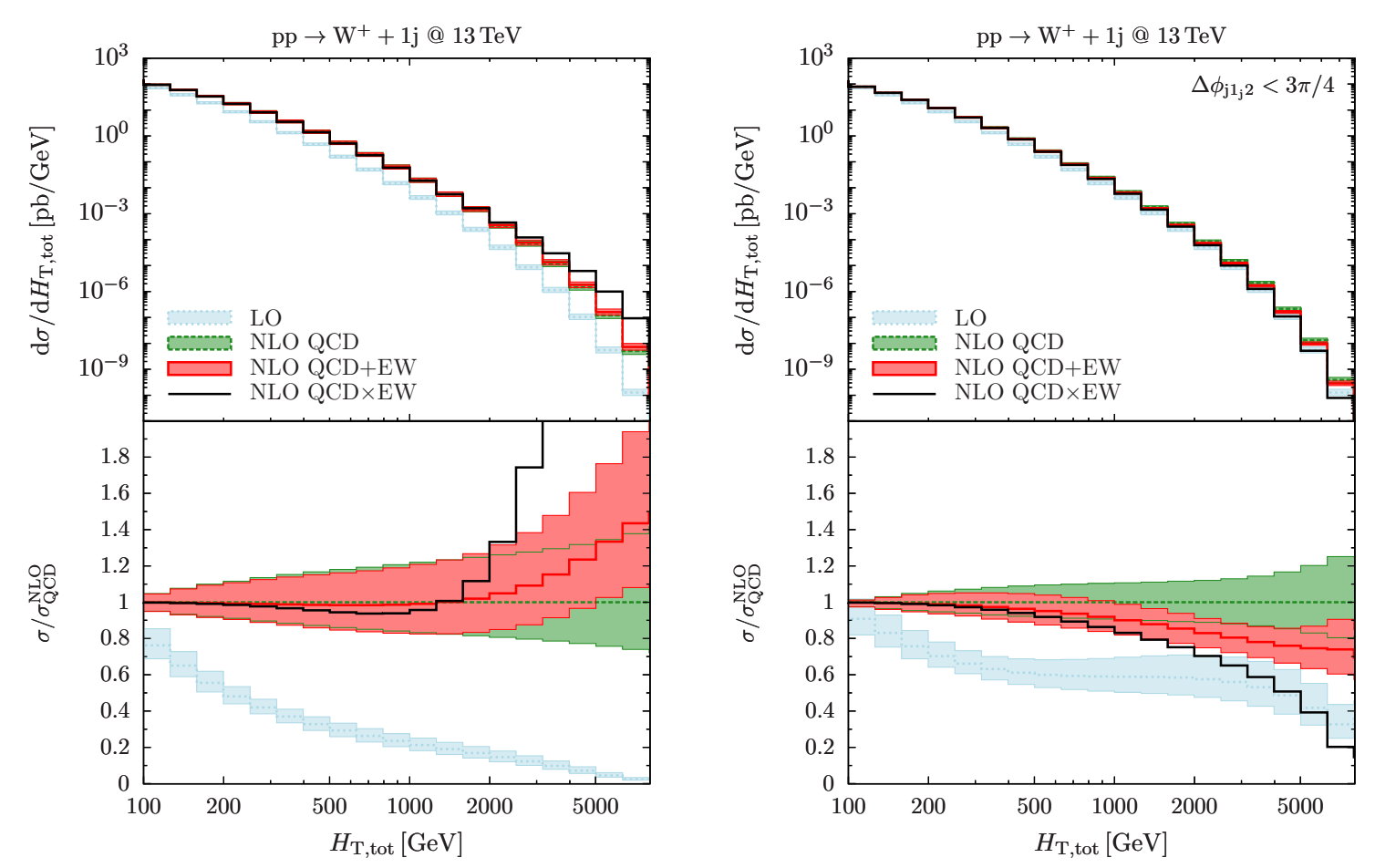

Figure 14. Distribution in $H_{\mathrm{T}}^{\text {tot }}$ for inclusive (left) $W^{+}+1 j$ production and with a cut $\Delta \phi_{j_{1} j_{2}}<3 \pi / 4$ (right). Curves and bands as in figure 13 .

As can be seen in the right plot of figure 13, in presence of the cut on $\Delta \phi_{j j}$, the improved perturbative QCD convergence leads to a consistent Sudakov behaviour for the NLO EW corrections to the $W$ - and jet- $p_{\mathrm{T}}$ distributions. These two observables behave in a quite similar way, as expected for exclusive $W+1 j$ events, where the jet and the $W$ boson recoil against each other, and the size of the corrections is around $-40 \%$ at $p_{\mathrm{T}}=2 \mathrm{TeV}$. Note that, in presence of the cut on $\Delta \phi_{j j}$, EW corrections exceed NLO QCD scale variations already at $p_{\mathrm{T}} \sim 200 \mathrm{GeV}$. The gap between the $\mathrm{EW}+\mathrm{QCD}$ and $\mathrm{EW} \times \mathrm{QCD}$ curves completely disappears in the case of the jet- $p_{\mathrm{T}}$ distribution, while for the $W$-boson $p_{\mathrm{T}}$ it remains problematic, due to the persistence of sizable QCD effects.

The distribution in $H_{\mathrm{T}}^{\text {tot}}$, shown in figure 14 , behaves in a qualitatively similar way as the jet- $p_{\mathrm{T}}$ distribution. However, also in presence of the $\Delta \phi_{j j}$ cut, this observable remains very sensitive to NLO QCD radiation, and the $\mathrm{QCD} \times \mathrm{EW}$ curve indicates that the observed $\mathrm{NLO} \mathrm{QCD}+\mathrm{EW}$ correction of $-25 \%$ at $H_{\mathrm{T}}^{\text {tot }}=4 \mathrm{TeV}$ might be underestimated by up to a factor two.

In summary, the strong sensitivity of $W+1$ jet production to NLO QCD real emission, which is effectively described at LO accuracy, leads to a sizable scale dependence and to an underestimate of EW correction effects in various observables. This calls for the calculation of NLO QCD+EW corrections to $W+2 j$ and $W+3 j$ production that we are going to present in the following sections. Numerical results for $p p \rightarrow W^{+}+1 j$ cross sections with different cuts are collected in table 2 . 


\begin{tabular}{|lllll|}
\hline$W^{+}+2 j$ & inclusive & $H_{\mathrm{T}}^{\text {tot }}>2 \mathrm{TeV}$ & $p_{\mathrm{T}, j_{1}}>1 \mathrm{TeV}$ & $p_{T, W^{+}}>1 \mathrm{TeV}$ \\
\hline$\sigma_{\mathrm{QCD}}^{\mathrm{NLO}}[\mathrm{pb}]$ & $4349_{-4 \%}^{+0 \%}$ & $0.364_{-13 \%}^{+12 \%}$ & $0.202_{-6 \%}^{+2 \%}$ & $0.048_{-7 \%}^{+0 \%}$ \\
$\sigma_{\mathrm{QCD}+\mathrm{EW}}^{\mathrm{NLO}}[\mathrm{pb}]$ & $4316_{-4 \%}^{+1 \%}$ & $0.329_{-10 \%}^{+8 \%}$ & $0.173_{-9 \%}^{+19 \%}$ & $0.033_{-25 \%}^{+6 \%}$ \\
$\sigma_{\mathrm{QCD}+\mathrm{EW}}^{\mathrm{NLO}} / \sigma_{\mathrm{QCD}}^{\mathrm{NLO}}$ & $0.99_{-4 \%}^{+1 \%}$ & $0.90_{-10 \%}^{+8 \%}$ & $0.86_{-9 \%}^{+1 \%}$ & $0.69_{-25 \%}^{+6 \%}$ \\
$\sigma_{\mathrm{QCD} \times \mathrm{EW}}^{\mathrm{NLO}} / \sigma_{\mathrm{QCD}}^{\mathrm{NLO}}$ & $0.99_{-4 \%}^{+1 \%}$ & $0.85_{-11 \%}^{+10 \%}$ & $0.84_{-4 \%}^{+0 \%}$ & $0.71_{-8 \%}^{+0 \%}$ \\
$\sigma^{\mathrm{LO}} / \sigma_{\mathrm{QCD}}^{\mathrm{NLO}}$ & $0.92_{-17 \%}^{+24 \%}$ & $0.64_{-23 \%}^{+31 \%}$ & $0.90_{-23 \%}^{+32 \%}$ & $1.05_{-23 \%}^{+33 \%}$ \\
\hline
\end{tabular}

Table 3. Integrated $p p \rightarrow W^{+}+2 j$ cross sections with inclusive cuts (6.1) and in presence of additional cuts. Born cross sections $\left(\sigma^{\mathrm{LO}}\right)$ include only the leading QCD contributions of $\mathcal{O}\left(\alpha_{\mathrm{S}}^{2} \alpha\right)$.

\section{$6.2 W^{+}+2$ jets}

Distributions and integrated cross sections for $p p \rightarrow W^{+}+2 j$ are presented in figures $15-17$ and table 3 , respectively. When the $W$ boson is accompanied by two jets, all one-particle inclusive $p_{\mathrm{T}}$ distributions, shown in the left plot of figure 15, are quite stable with respect to NLO QCD corrections. Scale uncertainties at NLO QCD are generally very small, and even in the tails they hardly exceed $10 \%$. The NLO EW corrections show a standard Sudakov behaviour and exceed NLO QCD uncertainties already at a few hundred GeV. For the $W$ boson $p_{\mathrm{T}}$ distribution they behave very similarly as in the case of $p p \rightarrow W+1 j$, reaching $-40 \%$ at $2 \mathrm{TeV}$. The EW corrections to the jet- $p_{\mathrm{T}}$ distributions are significantly smaller. At $2 \mathrm{TeV}$ they are around $-20 \%$, both for the first and for the second jet. Moreover, in the tails of the jet- $p_{\mathrm{T}}$ distributions, the trend of increasingly negative Sudakov corrections gets suppressed due to positive contributions from mixed EW-QCD bremsstrahlung, which result from interferences between diagrams of type 11a and 11c.

In figure 15 , results for inclusive $p_{\mathrm{T}}$ distributions (left) are compared to the same observables in presence of a cut $H_{\mathrm{T}}^{\text {tot }}>2 \mathrm{TeV}$ (right). The region of high $H_{\mathrm{T}}^{\text {tot }}$ plays a central role for BSM searches, and the upper right plot in figure 15 provides insights into the interplay between $W$-boson and jet transverse momenta in this kinematic region. The interesting part of the plot is the $p_{\mathrm{T}}$-range below $H_{\mathrm{T} \text {, cut }}^{\text {tot }} / 2$, where the $H_{\mathrm{T}}^{\text {tot }}$ cut is not trivially fulfilled, and the distributions behave in a significantly different way from the inclusive case. The shape of the various distributions can be understood in terms of a hard- $W$ regime where the $W$ boson carries $p_{\mathrm{T}} \gtrsim H_{\mathrm{T}, \text { cut }}^{\text {tot }} / 2$ and recoils against all jets - and a soft- $W$ regime - where the hardness of the event is driven by two back-to-back jets with $p_{\mathrm{T}} \gtrsim H_{\mathrm{T}, \text { cut }}^{\text {tot }} / 2$. The transition between these two regimes is controlled by the $W$-boson $p_{\mathrm{T}}$, whose distribution features a sharp change around $p_{\mathrm{T}, W}=H_{\mathrm{T}, \text { cut }}^{\text {tot }} / 2$. When the $W$-boson $p_{\mathrm{T}}$ enters the region below $H_{\mathrm{T}, \text { cut }}^{\text {tot }} / 2$ and approaches the soft regime, we observe that the growth of the cross section is drastically reduced as compared to the hard- $W$ regime. This indicates that, at large $H_{\mathrm{T}}^{\text {tot }}$, hard dijet signatures with soft $W$ bosons tend to be favoured, but $W$-boson emissions are distributed in a rather smooth way from low to high $p_{\mathrm{T}}$. This is consistent with the flatness of the $p_{\mathrm{T}}$-distribution of the second jet, which shares the first-jet recoil with the 

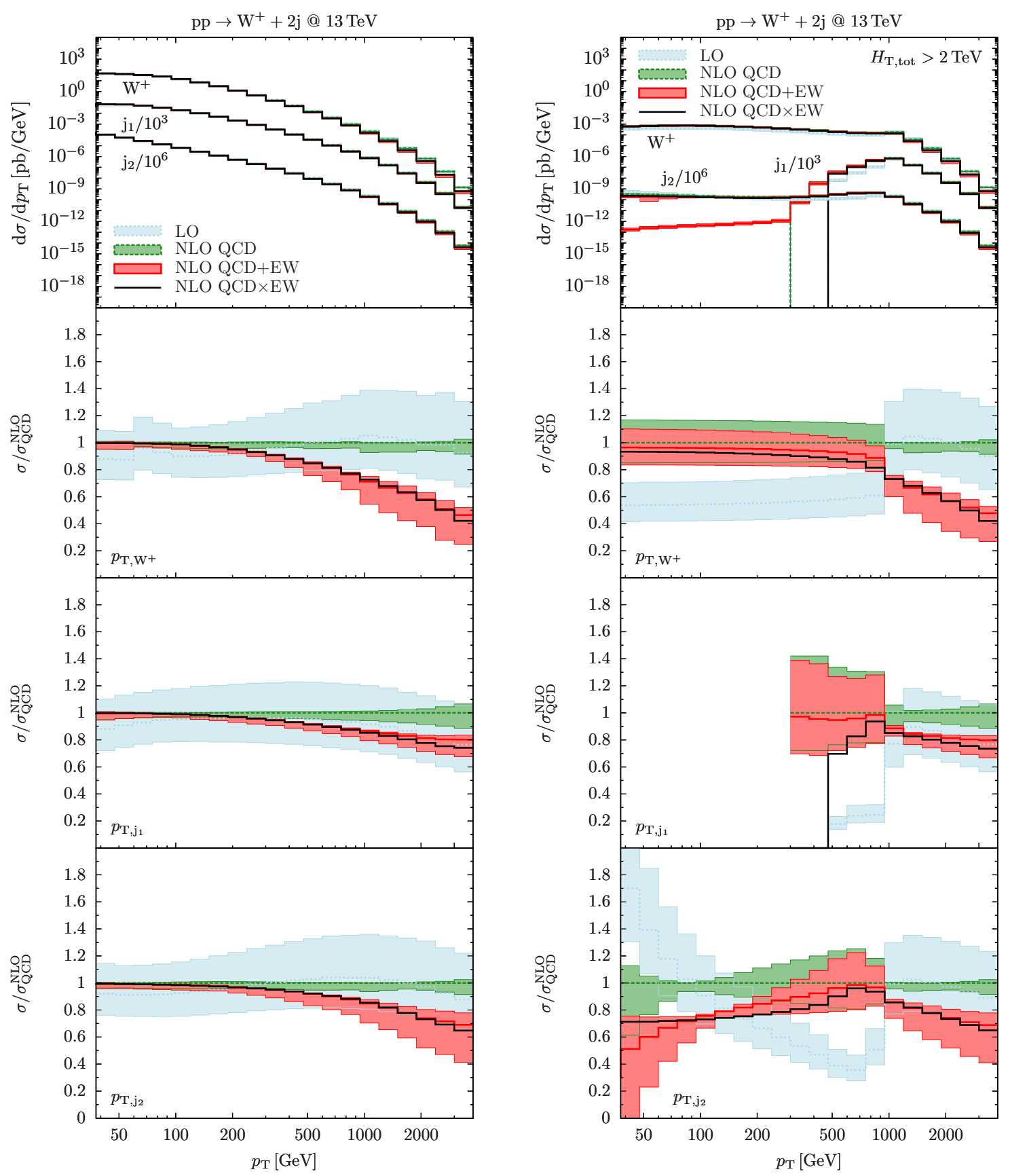

Figure 15. Distributions in the transverse momenta of the $W$ boson and the first two jets for inclusive (left) $W^{+}+2 j$ production and with a cut $H_{\mathrm{T}}^{\text {tot }}>2 \mathrm{TeV}$ (right). The distributions in the $n$-th jet $p_{\mathrm{T}}$ are rescaled by factors $10^{-3 n}$. Curves and bands as in figure 13 . 

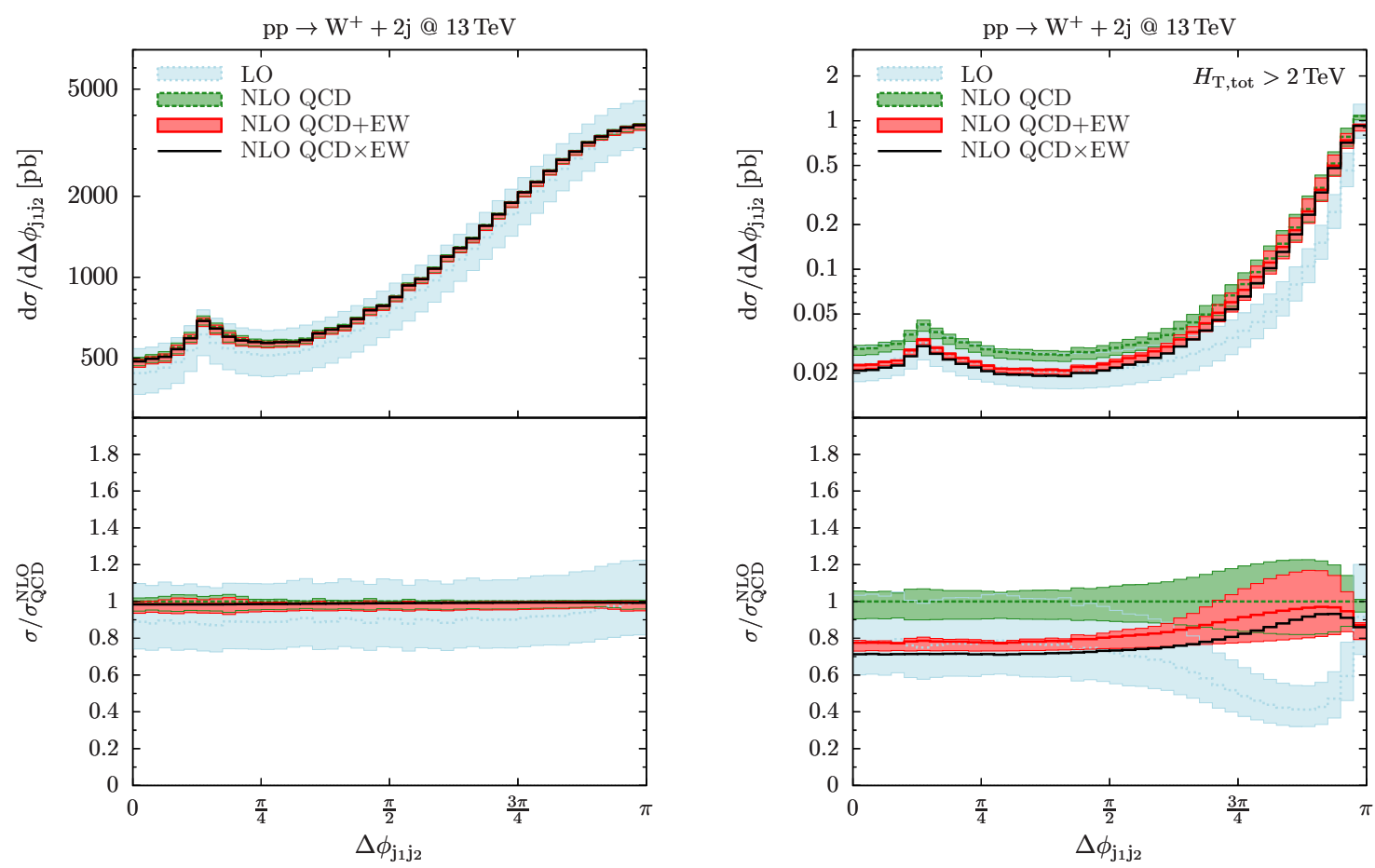

Figure 16. Distributions in the azimuthal separation of the first two jets for inclusive (left) $W^{+}+2 j$ production and with a cut $H_{\mathrm{T}}^{\text {tot }}>2 \mathrm{TeV}$ (right). Curves and bands as in figure 13 .

$W$ boson when the latter is not the hardest object. As for the first-jet $p_{\mathrm{T}}$, the peak at $p_{\mathrm{T}}=$ $H_{\mathrm{T}, \text { cut }}^{\text {tot }} / 2$ indicates that the cross section is dominated by events where the $W$ boson and the second jet recoil against the first jet, while the region $H_{\mathrm{T}, \text { cut }}^{\text {tot }} / 4<p_{\mathrm{T}}<H_{\mathrm{T}, \mathrm{tut}}^{\text {tot }} / 2$ corresponds to the hard- $W$ regime, where the two jets recoil against a $W$ boson with $p_{\mathrm{T}}>H_{\mathrm{T}}^{\text {tot }} / 2$.

For what concerns the behaviour of NLO corrections, we observe that, in contrast to the inclusive case, in the $p_{\mathrm{T}}$-region sensitive to the $H_{\mathrm{T} \text {, cut }}^{\text {tot }}$, i.e. below $H_{\mathrm{T}, \text { cut }}^{\text {tot }} / 2$, the various $p_{\mathrm{T}}$ distributions involve very strong NLO QCD corrections of $\mathcal{O}(100 \%)$ and correspondingly large NLO scale uncertainties. This can be attributed to the fact that the cut on $H_{\mathrm{T}}^{\text {tot }}$ can be efficiently saturated through QCD real-emission processes. Again, the fact that extra jet emission is effectively described at LO accuracy leads to underestimated EW correction effects. These features are clearly visible in the transition region around $H_{\mathrm{T}, \text { cut }}^{\text {tot }} / 2$, where the relative $\mathrm{QCD}+\mathrm{EW}$ correction jumps by about a factor two as compared to the smooth $\mathrm{QCD} \times \mathrm{EW}$ prediction. Apart from the problematic interplay of NLO QCD and EW corrections, the latter grow continuously with $p_{\mathrm{T}, W}$ as expected from EW Sudakov logarithms. The opposite trend in the second-jet $p_{\mathrm{T}}$ distribution is due to the fact that, below $H_{\mathrm{T}, \text { cut }}^{\text {tot }} / 2$, large $p_{\mathrm{T}, j_{2}}$ corresponds to small $p_{\mathrm{T}, W}$ and vice versa. Let us point out that the behaviour of the $W$-boson $p_{\mathrm{T}}$ distribution in the right plot of figure 15 is relevant for BSM searches that require very large $H_{\mathrm{T}}^{\text {tot }}$ without a correspondingly high cut on the leptonic and/or missing transverse energy. In this case, the $W+2 j$ background is clearly dominated by the region $p_{\mathrm{T}, W}<H_{\mathrm{T} \text {,cut }}^{\text {tot }} / 2$, where the $H_{\mathrm{T}}^{\text {tot }}$ cut leads to a bad perturbative QCD behaviour. This calls for higher-order corrections to $W+3 j$ production and, ultimately, for matching to the parton shower and multi-jet merging at NLO. 

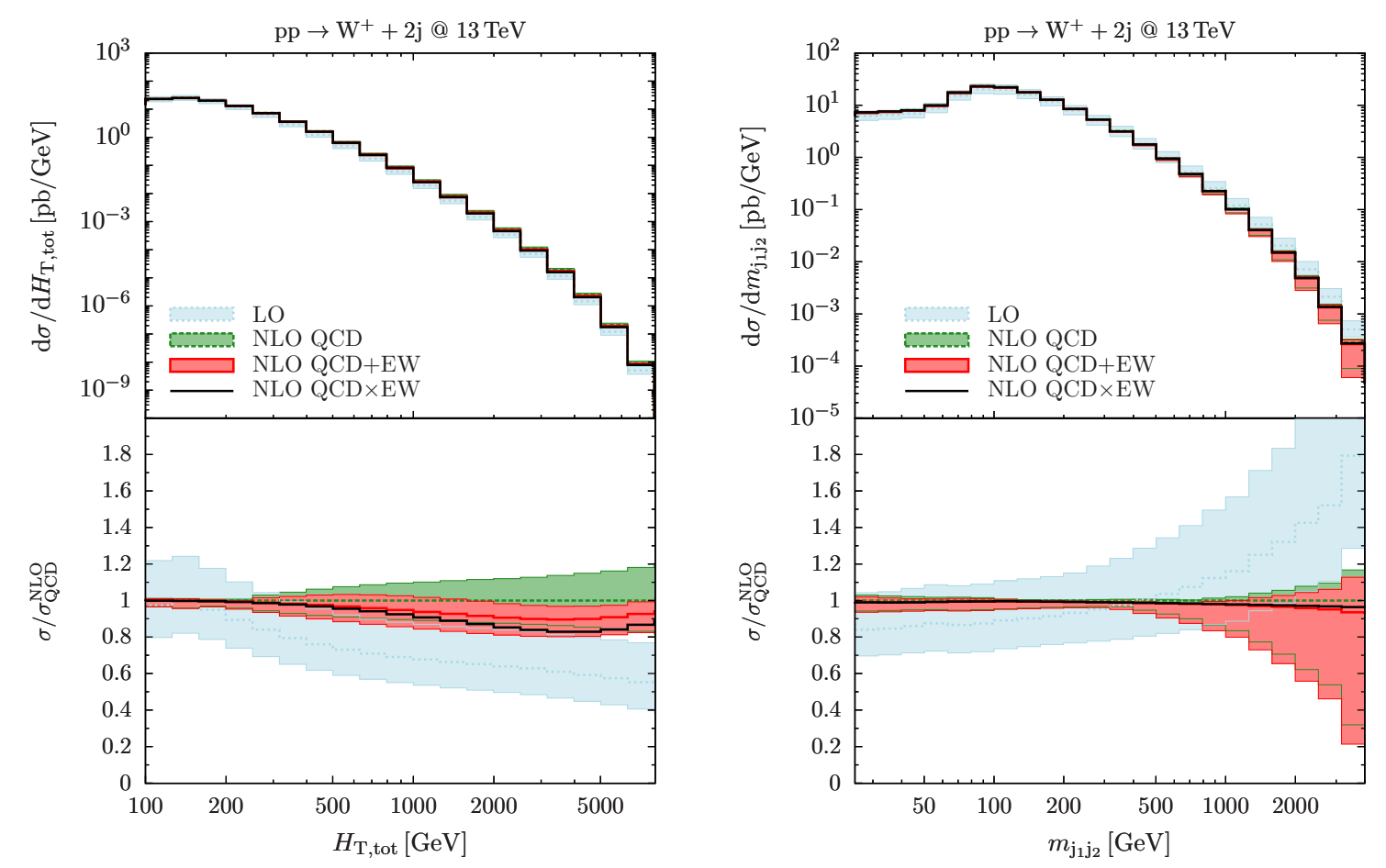

Figure 17. Distributions in $H_{\mathrm{T}}^{\text {tot }}$ (left) and in the invariant mass of the first two jets (right) for inclusive $W^{+}+2 j$ production. Curves and bands as in figure 13 .

Figure 16 presents the distribution in the azimuthal separation between the first two jets, $\Delta \phi_{j_{1} j_{2}}$, i.e. the variable used to isolate hard dijet configurations in section 6.1. The left plot shows that inclusive $W^{+}+2$-jet production is dominated by back-to-back dijet configurations, while the collinear peak around $\pi / 6$ remains clearly subdominant. For such an inclusive observable, NLO EW corrections are essentially negligible, and QCD corrections are rather small and stable. In presence of a cut $H_{\mathrm{T}}^{\text {tot }}>2 \mathrm{TeV}$ (right plot), $\Delta \phi_{j_{1} j_{2}}$ allows to discriminate the hard- $W$ regime - where all jets are in the hemisphere opposite to the $W$ boson — from the soft- $W$ regime - where the jets are back-to-back. As expected, the largest EW corrections are observed in the hard- $W$ regime (small $\Delta \phi_{j_{1} j_{2}}$ ), where they amount to about $-20 \%$, consistently with the inclusive result at $p_{\mathrm{T}}=1 \mathrm{TeV}$. In the soft- $W$ regime (large $\Delta \phi_{j_{1} j_{2}}$ ) NLO EW effects are much less pronounced. This is in part due to the fact that hard jets receive smaller EW Sudakov corrections as compared to hard $W$ bosons. Moreover, the presence of $\mathcal{O}(100 \%)$ QCD corrections induces a further strong suppression of NLO EW effects in this region.

The distribution in $H_{\mathrm{T}}^{\mathrm{tot}}$, displayed in figure 17, provides further evidence of the poor stability of this observable with respect to QCD radiation effects. In the tail, NLO QCD and EW corrections approach the $100 \%$ and $10 \%$ level, respectively, and the QCD $\times \mathrm{EW}$ curve suggests that the importance of NLO EW corrections is underestimated by a factor 2 in the NLO QCD+EW prediction. Finally, the distribution in the invariant mass of the first two jets (figure 17, right) behaves in a very different way: NLO EW corrections turn out to be very small and almost completely independent of the dijet mass, even in 


\begin{tabular}{|lllll|}
\hline$W^{+}+3 j$ & inclusive & $H_{\mathrm{T}}^{\text {tot }}>2 \mathrm{TeV}$ & $p_{\mathrm{T}, j_{1}}>1 \mathrm{TeV}$ & $p_{T, W^{+}}>1 \mathrm{TeV}$ \\
\hline$\sigma_{\mathrm{QCD}}^{\mathrm{NLO}}[\mathrm{pb}]$ & $1135_{-11 \%}^{+1 \%}$ & $0.377_{-12 \%}^{+0 \%}$ & $0.161_{-35 \%}^{+6 \%}$ & $0.038_{-14 \%}^{+0 \%}$ \\
$\sigma_{\mathrm{QCD}+\mathrm{EW}}^{\mathrm{NLO}}[\mathrm{pb}]$ & $1120_{-12 \%}^{+1 \%}$ & $0.313_{-26 \%}^{+3 \%}$ & $0.123_{-65 \%}^{+20 \%}$ & $0.026_{-40 \%}^{+8 \%}$ \\
$\sigma_{\mathrm{QCD}+\mathrm{EW}}^{\mathrm{NLO}} / \sigma_{\mathrm{QCD}}^{\mathrm{NLO}}$ & $0.99_{-12 \%}^{+1 \%}$ & $0.83_{-26 \%}^{+3 \%}$ & $0.76_{-65 \%}^{+20 \%}$ & $0.69_{-40 \%}^{+8 \%}$ \\
$\sigma_{\mathrm{QCD} \times \mathrm{EW}}^{\mathrm{NLO}} / \sigma_{\mathrm{QCD}}^{\mathrm{NLO}}$ & $0.99_{-11 \%}^{+1 \%}$ & $0.84_{-14 \%}^{+1 \%}$ & $0.83_{-37 \%}^{+9 \%}$ & $0.72_{-14 \%}^{+1 \%}$ \\
$\sigma^{\mathrm{LO}} / \sigma_{\mathrm{QCD}}^{\mathrm{NLO}}$ & $1.02_{-26 \%}^{+40 \%}$ & $1.05_{-28 \%}^{+42 \%}$ & $1.43_{-28 \%}^{+42 \%}$ & $1.09_{-28 \%}^{+43 \%}$ \\
\hline
\end{tabular}

Table 4. Integrated $p p \rightarrow W^{+}+3 j$ cross sections with inclusive cuts (6.1) and in presence of additional cuts. Born cross sections $\left(\sigma^{\mathrm{LO}}\right)$ include only the leading QCD contributions of $\mathcal{O}\left(\alpha_{\mathrm{S}}^{3} \alpha\right)$.

the multi- $\mathrm{TeV}$ range. This is explained by the fact that, in absence of an explicit high- $p_{\mathrm{T}}$ requirement, the region of large dijet mass is dominated by $t$-channel production at small $p_{\mathrm{T}}$. Note that in the tail of the $m_{j_{1} j_{2}}$ distribution QCD corrections become large, which results in sizable scale uncertainties.

In summary, NLO QCD+EW predictions for $p p \rightarrow W+2 j$ show a significantly improved perturbative stability as compared to the $W+1 j$ case. Nevertheless, the strong sensitivity of certain observables - in particular $H_{\mathrm{T}}^{\text {tot }}$ - to NLO QCD radiation calls for the extension of NLO QCD+EW calculations to $W+3$-jet production.

\section{3 $W^{+}+3$ jets}

Numerical results for $p p \rightarrow W^{+}+3 j$ at NLO QCD+EW are presented in figures 18-20 and in table 4 . At variance with the $W+2 j$ case, for one-particle inclusive $p_{\mathrm{T}}$ distributions, shown in figure 18, we find stable NLO QCD predictions only for the $W$ boson and the third jet, while the distributions in the $p_{\mathrm{T}}$ of the first two jets receive sizable negative QCD corrections in the region around $1 \mathrm{TeV}$. This suggests that the QCD scale choice (5.2) might be suboptimal for $W+3 j$ final states, and, in order to achieve better perturbative convergence, alternative dynamical scales should be considered. For instance, figure 18 indicates that using $\mu_{0}=\hat{H}_{\mathrm{T}}$ instead of $\mu_{0}=\hat{H}_{\mathrm{T}} / 2$, which corresponds to the lower boundary of the LO uncertainty band, would already improve the convergence in a significant way. However, in this paper we will stick to the standard choice (5.2) that was used in the most recent ATLAS analysis [3], and we defer a detailed study of alternative scale choices to a future publication.

As far as predictions obtained at the central scale $\mu_{0}=\hat{H}_{\mathrm{T}} / 2$ are concerned, NLO EW corrections in figure 18 are well behaved: the tails of all $p_{\mathrm{T}}$ distributions feature the expected EW Sudakov suppression, and the quantitative impact of the corrections is rather consistent with what is observed in the $W+2 j$ case. For the first- and second-jet $p_{\mathrm{T}}$-distributions, the $\mathrm{QCD} \times \mathrm{EW}$ curve suggests that the $\mathrm{NLO} \mathrm{QCD}+\mathrm{EW}$ approximation might overestimate EW correction effects, as a result of the negative QCD corrections. For what concerns scale variations, the fact that NLO QCD and NLO EW corrections are both very large leads to a very strong scale dependence at high $p_{\mathrm{T}}$. This illustrates, once again, 
that the optimal convergence of QCD predictions plays a key role for the stability of NLO QCD+EW predictions.

A very good perturbative convergence is found in figure 19 for the $H_{\mathrm{T}}^{\text {tot }}$ distribution (left). In presence of three associated jets this important observable receives fairly small QCD corrections. The NLO QCD+EW approximation can thus be regarded as a reliable description of EW correction effects, which reach $-20 \%$ at $H_{\mathrm{T}}^{\text {tot }}=4 \mathrm{TeV}$. For the distribution in the invariant mass of the first two jets, shown in the right plot of figure 19, a similar picture as in the case of $W+2 j$ production emerges. In particular EW corrections remain negligible in the entire $m_{j_{1} j_{2}}$ range.

Also the distribution in the azimuthal angular separation of the two hardest jets, shown in figure 20, behaves in a fairly similar way as for $W+2 j$ production. In particular, a cut $H_{\mathrm{T}}^{\text {tot }}>2 \mathrm{TeV}$ (right plot) induces EW corrections around $-20 \%$ in the hard- $W$ regime (small $\Delta \phi_{j_{1} j_{2}}$ ) while in the soft- $W$ regime (large $\Delta \phi_{j_{1} j_{2}}$ ) NLO EW effects are clearly less pronounced.

Numerical results for $p p \rightarrow W^{+}+3 j$ cross sections with different cuts are collected in table 4 .

\subsection{Subleading and photon-induced Born contributions}

In the following we briefly discuss the numerical impact of subleading and photon-induced Born contributions to the production of a $W^{+}$boson in association with $n=1,2,3$ jets.

As discussed in section 4.3, the production of $W+2,3$ jets receives pure EW contributions of $\mathcal{O}\left(\alpha_{\mathrm{S}}^{n-2} \alpha^{3}\right)$ as well as contributions of $\mathcal{O}\left(\alpha_{\mathrm{S}}^{n-1} \alpha^{2}\right)$ from interferences of QCD- and EW-type diagrams in the four-quark channels. In addition, the production of $W+1,2,3$ jets can proceed through different $\gamma$-induced processes, as discussed in section 4.4. The pure EW contributions and the resonant $\gamma \gamma$-induced processes of pure EW-type at $\mathcal{O}\left(\alpha_{\mathrm{S}}^{n-2} \alpha^{3}\right)$ involve physical Z, W, and top-quark resonances, which are regularized by their corresponding physical decay widths. ${ }^{15}$ The impact of the resulting violation of gauge invariance due to the approximation of an on-shell $\mathrm{W}$ was found to be at the small percent level of the respective contribution. A consistent gauge-invariant treatment for these processes at NLO will require a full SM calculation with decays.

Results for integrated cross sections and distributions are listed in table 5 and figures 21-23, respectively. As far as subleading Born contributions are concerned, in the integrated $W+2,3$ jet cross sections EW-QCD mixed Born effects of $\mathcal{O}\left(\alpha_{\mathrm{S}}^{n-1} \alpha^{2}\right)$ are at the permil level, while the pure EW contributions of $\mathcal{O}\left(\alpha_{\mathrm{S}}^{n-2} \alpha^{3}\right)$ are one order of magnitude larger. This is due to the presence of resonances that correspond to di-boson and singletop production (with hadronic decays of a $W^{-}$or $Z$ boson). The relative importance of EW-QCD Born interference terms grows with the jet- $p_{\mathrm{T}}$, and at $1 \mathrm{TeV}$ these contributions reach $11 \%(14 \%)$ in $p p \rightarrow W^{+}+2 j(3 j)$. This enhancement can also be understood as a PDF effect where the contribution of the four-quark channel increases over the two-quark channel due to a relative increase of the quark PDFs over the gluon PDFs for large $x$. In certain phase-space regions, EW-QCD interference contributions become negative.

\footnotetext{
${ }^{15}$ We use the following values of the relevant particle widths, which are calculated at LO from the parameters stated in section $5, \Gamma_{\mathrm{W}}=2.04544 \mathrm{GeV}, \Gamma_{\mathrm{Z}}=2.44408 \mathrm{GeV}$, and $\Gamma_{\mathrm{t}}=1.50175 \mathrm{GeV}$.
} 


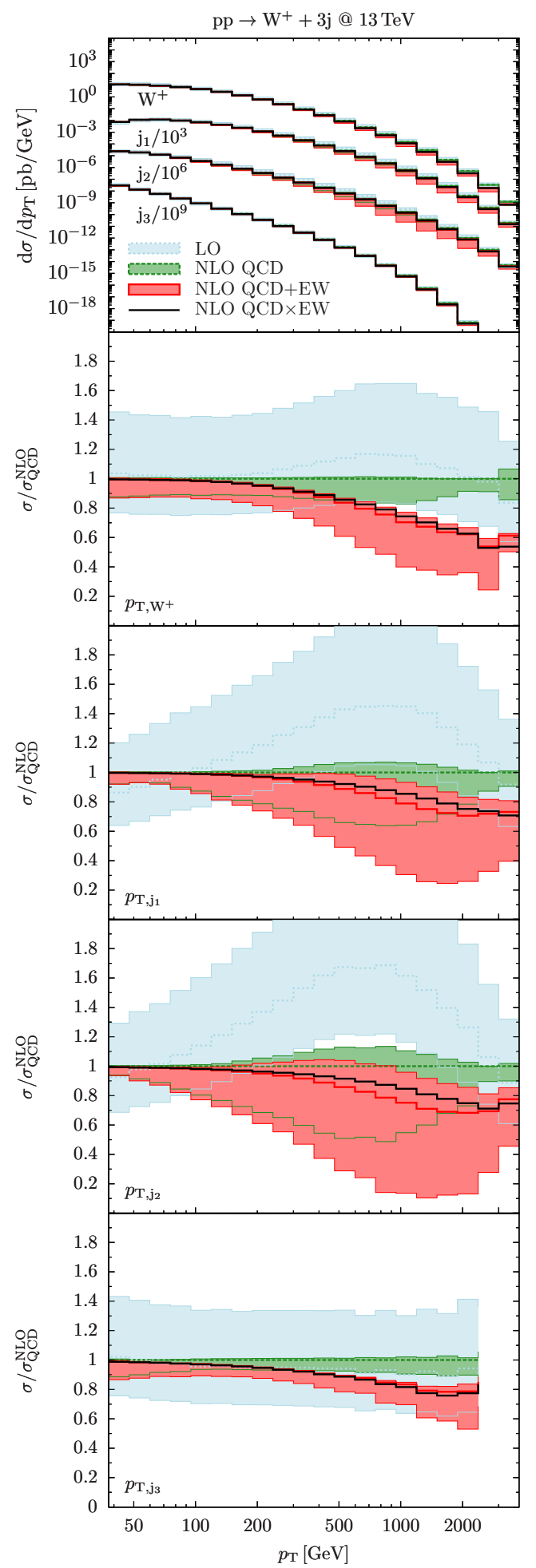

Figure 18. Distributions in the transverse momenta of the $W$ boson and of the first three jets for inclusive $W^{+}+3 j$ production. The distributions in the $n$-th jet $p_{\mathrm{T}}$ are rescaled by factors $10^{-3 n}$. Curves and bands as in figure 13 . 

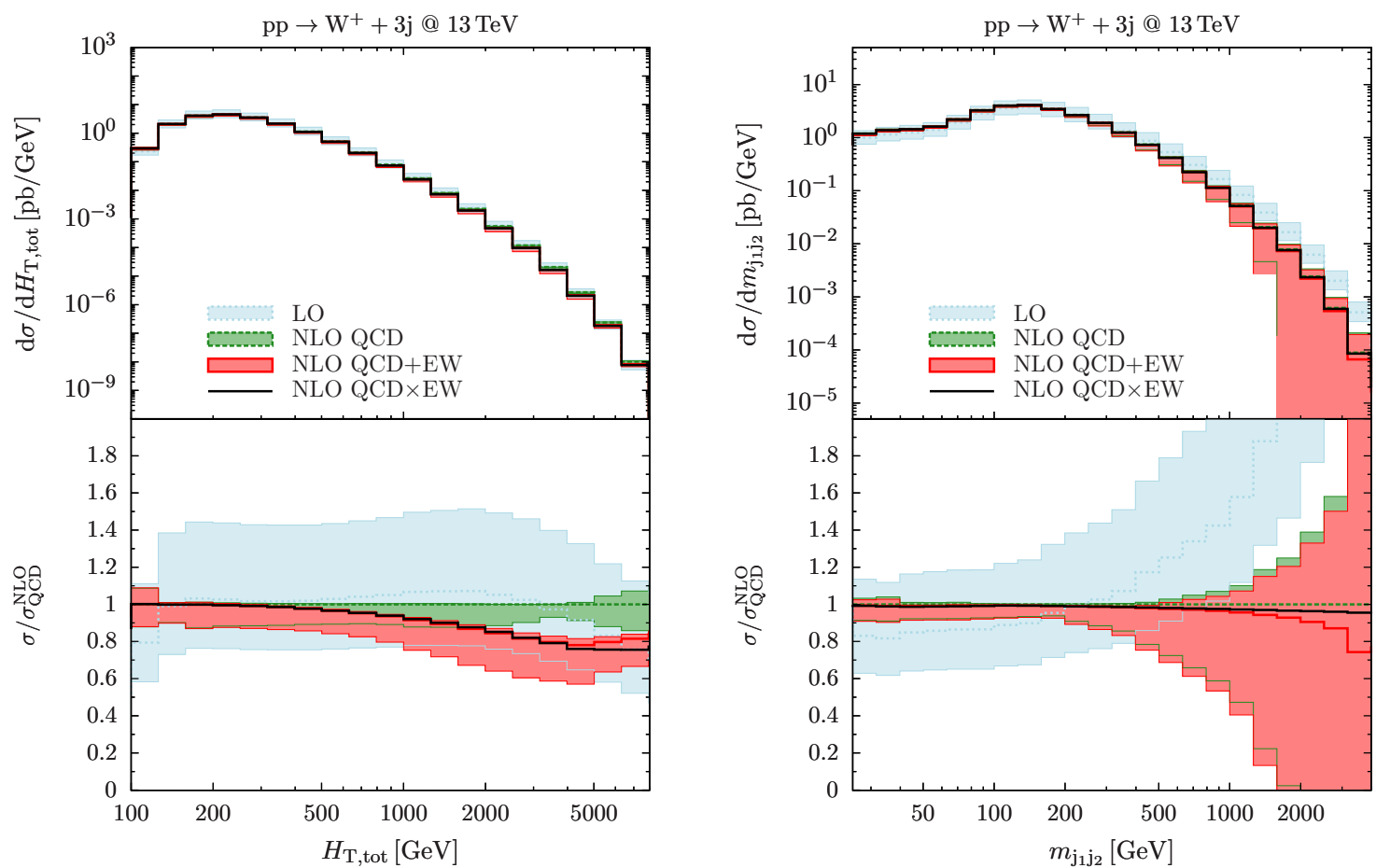

Figure 19. Distributions in $H_{\mathrm{T}}^{\text {tot }}$ (left) and in the invariant mass of the first two jets (right) for inclusive $W^{+}+3 j$ production. Curves and bands as in figure 13 .
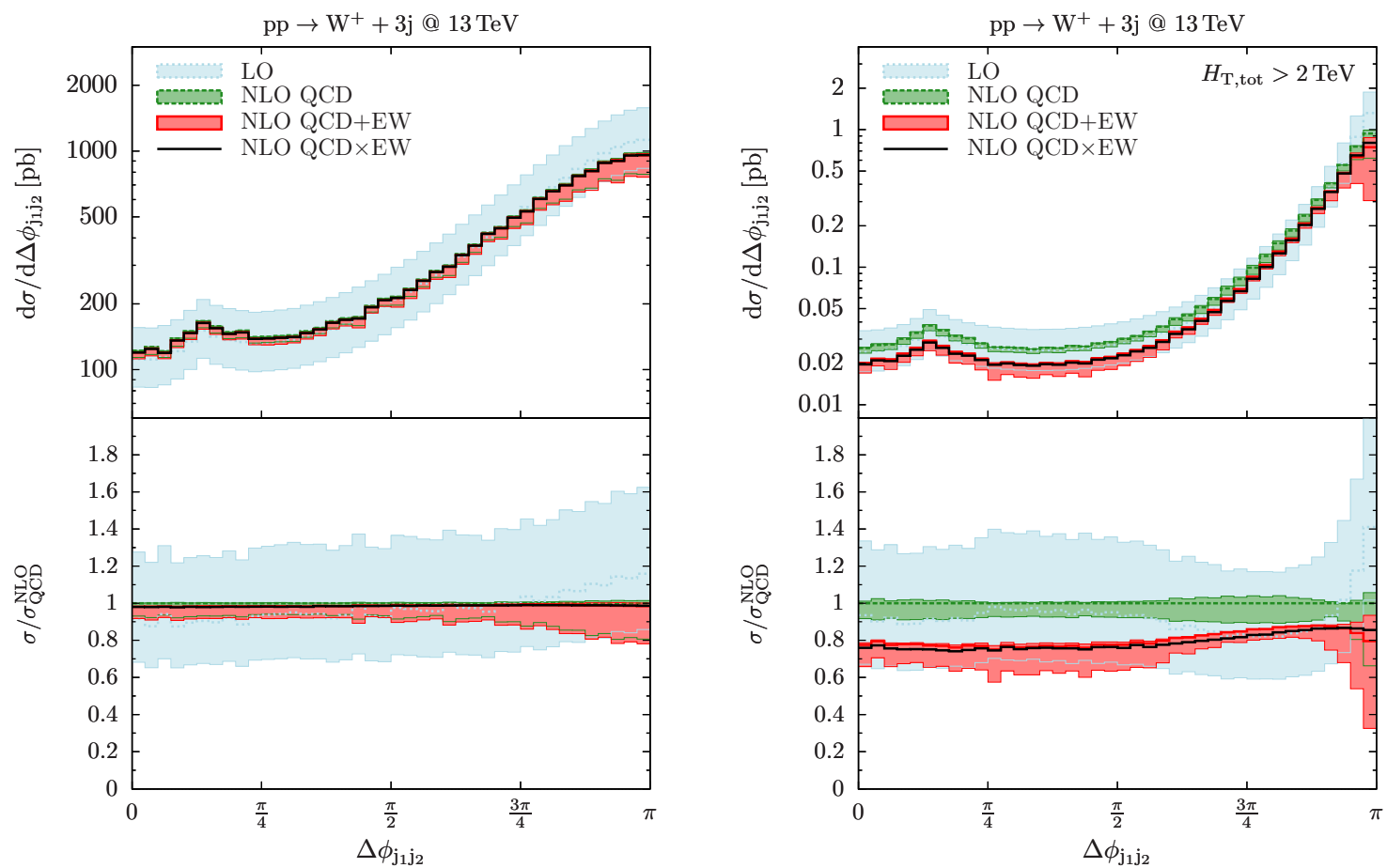

Figure 20. Distributions in the azimuthal separation of the first two jets for inclusive (left) $W^{+}+3 j$ production and with a cut $H_{\mathrm{T}}^{\text {tot }}>2 \mathrm{TeV}$ (right). Curves and bands as in figure 13 . 


\begin{tabular}{|clllll|}
\hline Born order & $W^{+}+1 j$ & inclusive & $H_{\mathrm{T}}^{\text {tot }}>2 \mathrm{TeV}$ & $p_{\mathrm{T}, j_{1}}>1 \mathrm{TeV}$ & $p_{T, W^{+}}>1 \mathrm{TeV}$ \\
\hline $\mathcal{O}\left(\alpha^{2}\right)$ & $\sigma_{\gamma p}^{\text {Born }} / \sigma_{\mathrm{QCD}}^{\mathrm{NLO}}$ & $0.0031_{-14 \%}^{+14 \%}$ & $0.0173_{-1 \%}^{+1 \%}$ & $0.0221_{-1 \%}^{+1 \%}$ & $0.0805_{-1 \%}^{+1 \%}$ \\
\hline Born order & $W^{+}+2 j$ & inclusive & $H_{\mathrm{T}}^{\text {tot }}>2 \mathrm{TeV}$ & $p_{\mathrm{T}, j_{1}}>1 \mathrm{TeV}$ & $p_{T, W^{+}}>1 \mathrm{TeV}$ \\
\hline $\mathcal{O}\left(\alpha_{\mathrm{S}} \alpha^{2}\right)$ & $\sigma_{p p}^{\text {Born }} / \sigma_{\mathrm{QCD}}^{\mathrm{NLO}}$ & $0.0008_{-11 \%}^{+14 \%}$ & $0.0659_{-15 \%}^{+19 \%}$ & $0.1085_{-15 \%}^{+19 \%}$ & $-0.0006_{+21 \%}^{-30 \%}$ \\
$\mathcal{O}\left(\alpha^{3}\right)$ & $\sigma_{p p}^{\text {Born }} / \sigma_{\mathrm{QCD}}^{\mathrm{NLO}}$ & $0.0345_{-8 \%}^{+6 \%}$ & $0.0562_{-10 \%}^{+11 \%}$ & $0.0792_{-10 \%}^{+11 \%}$ & $0.0728_{-10 \%}^{+12 \%}$ \\
$\mathcal{O}\left(\alpha_{\mathrm{S}} \alpha^{2}\right)$ & $\sigma_{\gamma p}^{\text {Born }} / \sigma_{\mathrm{QCD}}^{\mathrm{NLO}}$ & $0.0014_{-10 \%}^{+11 \%}$ & $0.0083_{-8 \%}^{+9 \%}$ & $0.0103_{-8 \%}^{+9 \%}$ & $0.0426_{-8 \%}^{+9 \%}$ \\
$\mathcal{O}\left(\alpha^{3}\right)$ & $\sigma_{\gamma \gamma}^{\text {Born }} / \sigma_{\mathrm{QCD}}^{\mathrm{NLO}}$ & $<0.0001$ & $<0.0001$ & $<0.0001$ & $0.0001_{-8 \%}^{+9 \%}$ \\
\hline $\mathrm{Born}$ order & $W^{+}+3 j$ & inclusive & $H_{\mathrm{T}}^{\text {tot }}>2 \mathrm{TeV}$ & $p_{\mathrm{T}, j_{1}}>1 \mathrm{TeV}$ & $p_{T, W^{+}}>1 \mathrm{TeV}$ \\
\hline $\mathcal{O}\left(\alpha_{\mathrm{S}}^{2} \alpha^{2}\right)$ & $\sigma_{p p}^{\text {Born }} / \sigma_{\mathrm{QCD}}^{\mathrm{NLO}}$ & $0.0018_{-17 \%}^{+23 \%}$ & $0.0796_{-21 \%}^{+28 \%}$ & $0.1351_{-21 \%}^{+28 \%}$ & $0.0016_{-30 \%}^{+43 \%}$ \\
$\mathcal{O}\left(\alpha_{\mathrm{S}} \alpha^{3}\right)$ & $\sigma_{p p}^{\text {Born }} / \sigma_{\mathrm{QCD}}^{\mathrm{NLO}}$ & $0.0619_{-8 \%}^{+10 \%}$ & $0.0670_{-16 \%}^{+21 \%}$ & $0.0947_{-16 \%}^{+21 \%}$ & $0.0831_{-17 \%}^{+22 \%}$ \\
$\mathcal{O}\left(\alpha_{\mathrm{S}}^{2} \alpha^{2}\right)$ & $\sigma_{\gamma p}^{\text {Born }} / \sigma_{\mathrm{QCD}}^{\mathrm{NLO}}$ & $0.0011_{-15 \%}^{+21 \%}$ & $0.0057_{-14 \%}^{+18 \%}$ & $0.0073_{-15 \%}^{+18 \%}$ & $0.0197_{-14 \%}^{+18 \%}$ \\
$\mathcal{O}\left(\alpha_{\mathrm{S}} \alpha^{3}\right)$ & $\sigma_{\gamma p}^{\text {Born }} / \sigma_{\mathrm{QCD}}^{\mathrm{NLO}}$ & $\lessgtr \pm 0.0001$ & $\lessgtr \pm 0.0001$ & $\lessgtr \pm 0.0001$ & $\lessgtr \pm 0.0001$ \\
$\mathcal{O}\left(\alpha^{4}\right)$ & $\sigma_{\gamma p}^{\text {Born }} / \sigma_{\mathrm{QCD}}^{\mathrm{NLO}}$ & $0.0014_{-15 \%}^{+15 \%}$ & $0.0013_{-2 \%}^{+2 \%}$ & $0.0018_{-2 \%}^{+2 \%}$ & $0.0057_{-2 \%}^{+2 \%}$ \\
$\mathcal{O}\left(\alpha_{\mathrm{S}} \alpha^{3}\right)$ & $\sigma_{\gamma \gamma}^{\text {Born }} / \sigma_{\mathrm{QCD}}^{\mathrm{NLO}}$ & $<0.0001$ & $<0.0001$ & $<0.0001$ & $<0.0001$ \\
\hline
\end{tabular}

Table 5. Integrated cross sections for $p p \rightarrow W^{+}+n$ jet production with $n=1,2,3$ with inclusive cuts (6.1) and in presence of additional cuts: $\mathcal{O}\left(\alpha_{\mathrm{S}}^{n-1} \alpha^{2}\right)$ mixed EW-QCD, $\mathcal{O}\left(\alpha_{\mathrm{S}}^{n-2} \alpha^{3}\right)$ pure EW, photon-proton induced Born contributions of $\mathcal{O}\left(\alpha_{\mathrm{S}}^{n-1} \alpha^{2}\right) \ldots \mathcal{O}\left(\alpha^{n+1}\right)$ and photon-photon induced contributions of $\mathcal{O}\left(\alpha_{\mathrm{S}}^{n-2} \alpha^{3}\right)$. The various contributions are normalised to corresponding NLO QCD predictions.

Photon-induced effects induce only permil-level contributions to the various inclusive cross sections. Still, the increasing importance of the photon density at high Bjorken $x$ leads to an enhancement of $\gamma$-induced cross sections at large $W$-boson transverse momenta. At $p_{\mathrm{T}, \mathrm{W}^{+}}>1 \mathrm{TeV}$, the dominant $\mathcal{O}\left(\alpha_{\mathrm{S}}^{n-1} \alpha^{2}\right)$ photon-induced contributions to $W^{+}+1,2,3$ jets are around $8 \%, 4 \%$, and $2 \%$, respectively, and their magnitude grows extremely rapidly up to $\mathcal{O}(100 \%)$ in the multi-TeV range. In this respect, one should keep in mind that the photon PDF is still very poorly constrained in this regime [68], and $W+$ jets measurements at large transverse momenta might provide useful input for a better determination of the photon PDF. We observe that $\gamma \gamma$-induced processes are strongly suppressed in the entire phase space. 


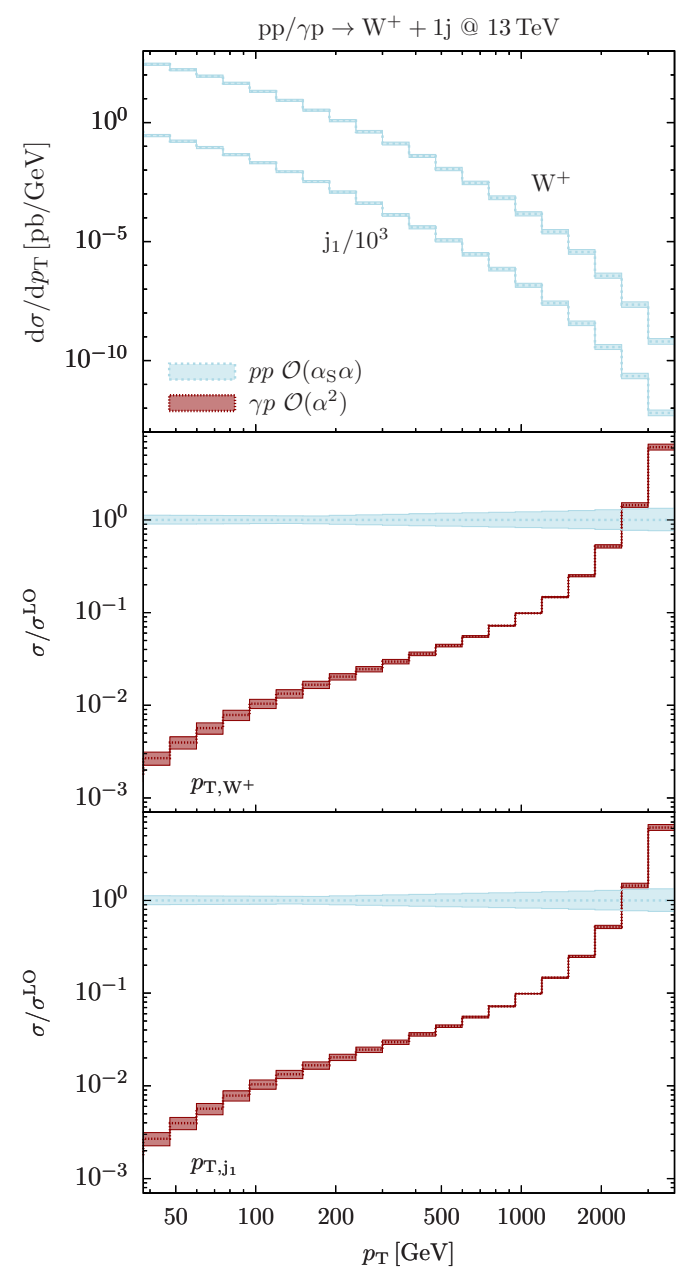

Figure 21. Distributions in the transverse momenta of the $W$ boson and of the first jet for inclusive $W^{+}+1 j$ production. In the upper panel absolute predictions for the LO Born contribution at $\mathcal{O}\left(\alpha_{\mathrm{S}} \alpha\right)$ (light blue) are shown. The distribution in $p_{\mathrm{T}, j_{1}}$ is rescaled by a factor $10^{-3}$. In the lower panels photon-proton induced predictions at $\mathcal{O}\left(\alpha^{2}\right)$ (dark red) are shown relative to the LO Born contribution. The bands correspond to scale variations, and in the case of ratios only the numerator is varied.

\section{$7 \quad$ Summary and conclusions}

The calculation of electroweak corrections is a central prerequisite for precision tests of the Standard Model and for new-physics searches at the energy frontier. In particular, the strong impact of EW corrections on a wide range of processes and observables at the $\mathrm{TeV}$ scale motivates the extension of automated NLO generators from the QCD to the EW sector of the Standard Model.

In this context, a systematic bookkeeping of all possible EW-QCD interference terms at NLO is needed. Standard NLO EW corrections of a certain order $\alpha_{\mathrm{S}}^{n} \alpha^{m+1}$ arise via insertion of virtual or real electroweakly interacting particles in squared tree amplitudes of order $g_{\mathrm{S}}^{n} e^{m}$. But NLO EW corrections at the same order $\alpha_{\mathrm{S}}^{n} \alpha^{m+1}$ can also arise via 

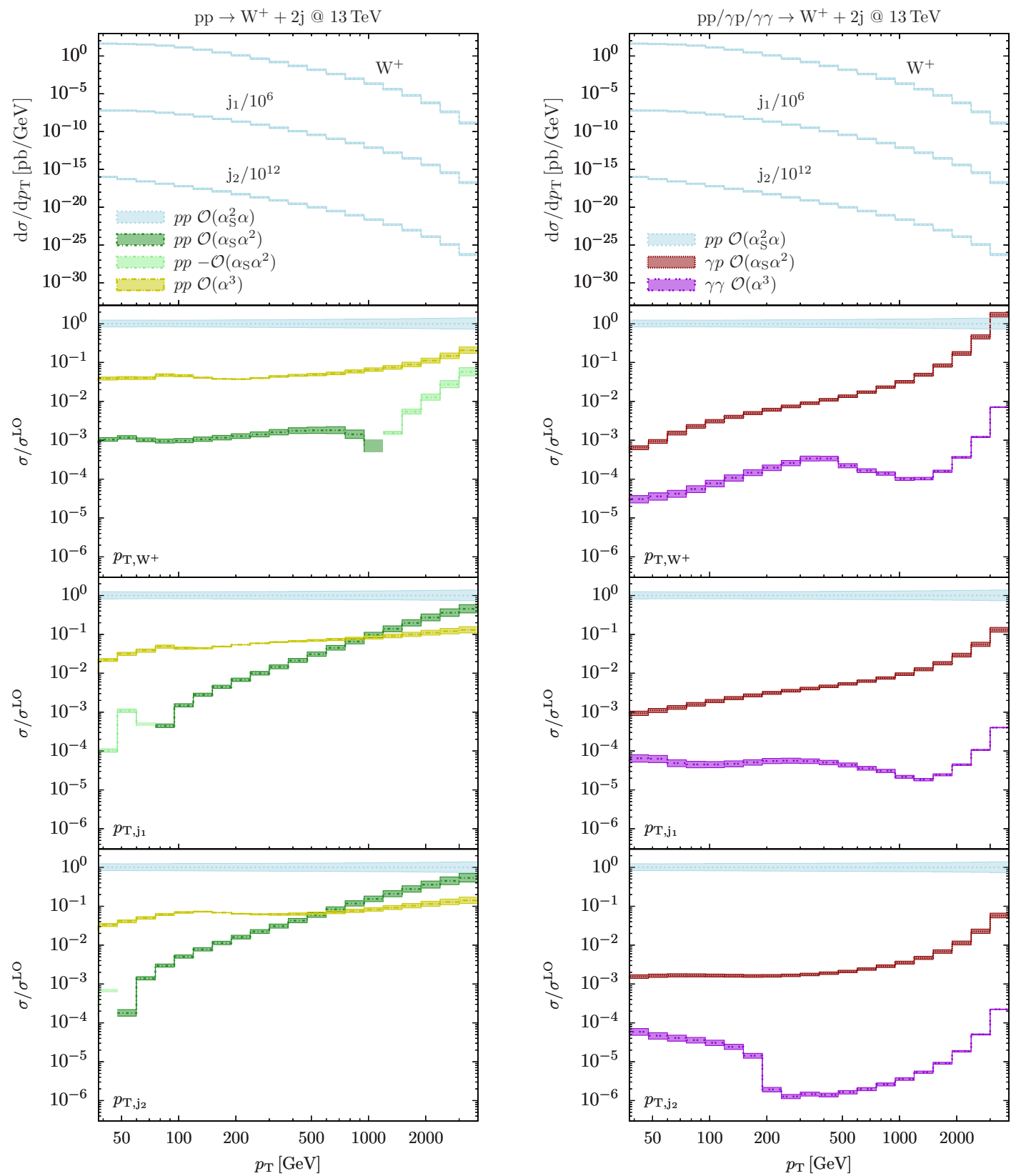

Figure 22. Distributions in the transverse momenta of the $W$ boson and of the first two jets for inclusive $W^{+}+2 j$ production. In the upper panels absolute predictions for the LO Born contribution at $\mathcal{O}\left(\alpha_{\mathrm{S}}^{2} \alpha\right)$ (light blue) are shown. The distributions in the $n$-th jet are rescaled by a factor $10^{-6 n}$. In the lower panels, on the left, proton-proton induced mixed EW-QCD predictions at $\mathcal{O}\left(\alpha_{\mathrm{S}} \alpha^{2}\right)$ (dark/light green depending on the sign) and resonant EW predictions at $\mathcal{O}\left(\alpha^{3}\right)$ (olive) relative to the LO Born contribution are shown. On the right, photon-proton and photon-photon induced predictions at $\mathcal{O}\left(\alpha_{\mathrm{S}} \alpha^{2}\right)$ (dark red) and $\mathcal{O}\left(\alpha^{3}\right)$ (violet), respectively, are shown relative to the LO Born contribution. Bands as in figure 21. 

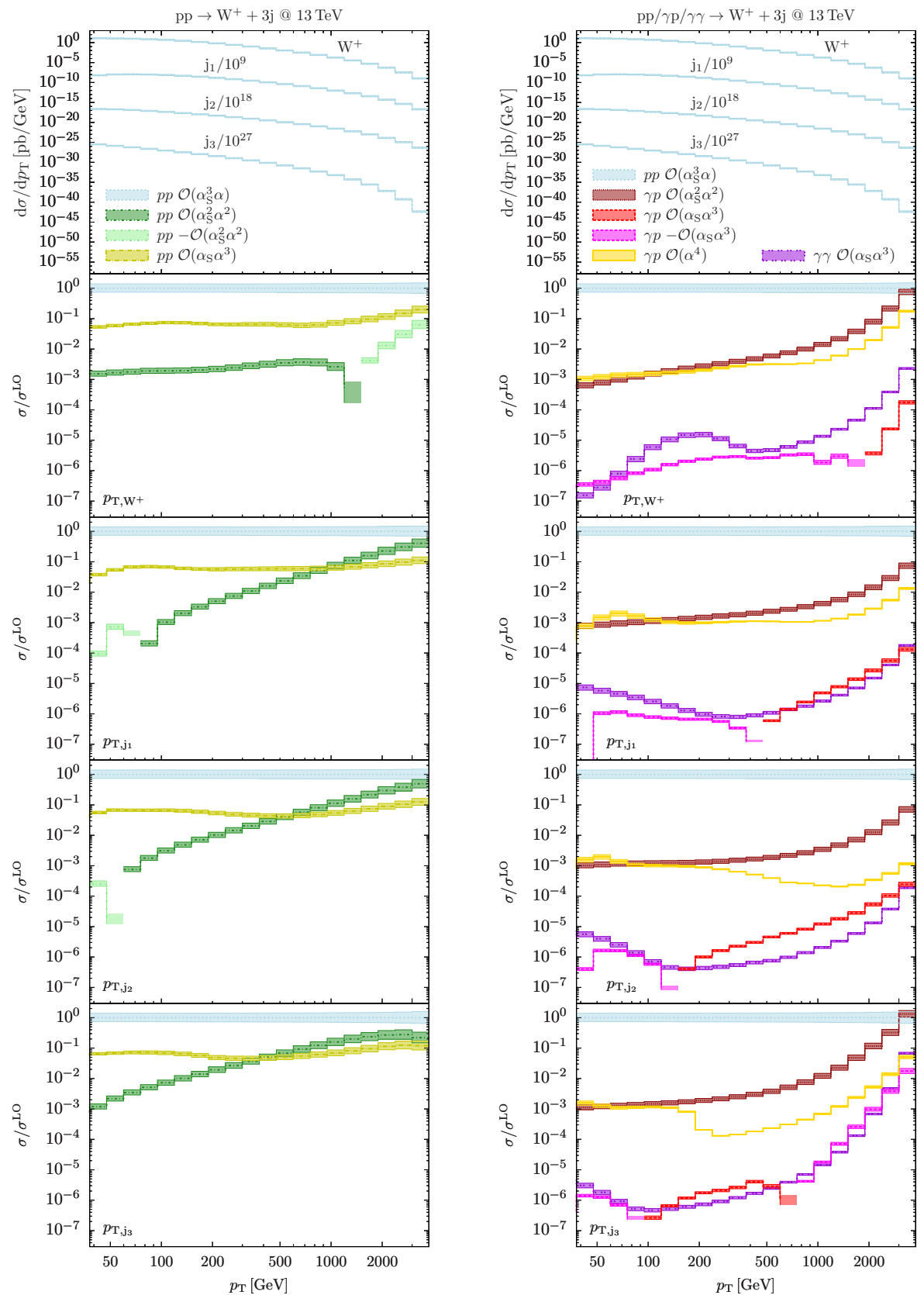

Figure 23. Distributions in the transverse momenta of the $W$ boson and of the first $n$ jets for inclusive $W^{+}+3 j$ production. In the upper panels absolute predictions for the LO Born contribution at $\mathcal{O}\left(\alpha_{\mathrm{S}}^{3} \alpha\right)$ (light blue) are shown. The distributions in the $n$-th jet are rescaled by a factor $10^{-9 n}$. In the lower panels, on the left, proton-proton induced mixed EW-QCD predictions at $\mathcal{O}\left(\alpha_{\mathrm{S}}^{2} \alpha^{2}\right)$ (dark/light green depending on the sign) and resonant EW predictions at $\mathcal{O}\left(\alpha_{\mathrm{S}} \alpha^{3}\right)$ (olive) relative to the LO Born contribution are shown. On the right, predictions for photon-proton induced production at $\mathcal{O}\left(\alpha_{\mathrm{S}}^{2} \alpha^{2}\right)$ (dark red), $\mathcal{O}\left(\alpha_{\mathrm{S}} \alpha^{3}\right)$ (red/magenta depending on the sign) and $\mathcal{O}\left(\alpha^{4}\right)$ (yellow) are shown together with photon-photon induced production at $\mathcal{O}\left(\alpha_{\mathrm{S}} \alpha^{3}\right)$ (violet) relative to the LO Born contribution. Bands as in figure 21. 
insertion of virtual or real strongly interacting partons in interference terms between tree amplitudes of order $g_{\mathrm{S}}^{n} e^{m}$ and $g_{\mathrm{S}}^{n-2} e^{m+2}$. In general, in order to obtain infrared-finite cross sections, all possible EW-QCD interference terms that contribute at a given order $\alpha_{\mathrm{S}}^{n} \alpha^{m+1}$ need to be included.

The cancellation of infrared singularities at NLO EW requires the real emission of QED and possibly also QCD partons, while the factorisation of initial-state collinear singularities requires QED effects in the PDFs, including a photon density. Moreover, due to the interplay of QED and QCD IR singularities associated with collinear photon-quark and photon-gluon pairs inside jets, at NLO EW the infrared-safe definition of jet observables and the separation of hard jets from hard photons is nontrivial. In this respect we have discussed a theoretical definition of jets based on democratic jet clustering in combination with a photon-jet separation formulated in terms of the photon-energy fraction inside jets. In particular, we have shown that the cancellation of QED and QCD infrared singularities can be achieved by a simple recombination prescription for photon-quark pairs in a way that provides an excellent approximation to a rigorous jet definition based on quark fragmentation functions.

The first key result presented in this paper is the complete automation of NLO $\mathrm{QCD}+\mathrm{EW}$ calculations within the OpEnLoops one-loop generator in combination with the Monte Carlo programs Munich and Sherpa. The OpenLoops program generates all relevant matrix-element ingredients, i.e. one-loop amplitudes, tree amplitudes for Born and bremsstrahlung contributions, as well as colour-, charge-, gluon-helicity and photon-helicity correlations that are needed for IR subtractions. Tree and one-loop matrix elements can be generated at any desired order $\alpha_{\mathrm{S}}^{n} \alpha^{m}$, including all relevant EW-QCD interferences, and full NLO Standard Model calculations are also possible. To automate one-loop EW calculations, all EW Feynman rules have been implemented in the framework of the numerical OpENLOOPS recursion and complemented by counterterms associated with $R_{2}$ rational parts and with the on-shell renormalisation of UV singularities.

All complementary tasks, i.e. the bookkeeping of partonic processes, the subtraction of IR singularities, and phase space integration, have been automated within MUNICH and Sherpa. These two alternative Monte Carlo frameworks are based on the dipolesubtraction formalism, whose implementation had to be extended from NLO QCD to NLO QED. In combination with OPENLoops, these tools automate the full chain of operations - from process definition to collider observables - that enter NLO QCD+EW simulations at parton level. As far as the efficiency of the simulations is concerned, the fact that OpenLoops can evaluate one-loop EW matrix elements at a similarly high speed as in the QCD case opens the route to NLO QCD+EW studies for a very wide range of processes, up to high particle multiplicity.

As a first nontrivial application, we have presented NLO QCD+EW predictions for $W$-boson production in association with one, two, and three jets at the $13 \mathrm{TeV}$ LHC. This represents the first NLO EW calculation for an LHC process with more than two jets and for $W+n$-jet production with $n=2$ and $n=3$. Since the $\mathrm{EW}$ corrections to $W+$ jets production are expected to be almost independent of the $W$-boson charge [34], we have restricted ourselves to the case of positively charged $W$ bosons. Our predictions include 
all $\mathcal{O}\left(\alpha_{\mathrm{S}}^{n+1} \alpha\right)$ and $\mathcal{O}\left(\alpha_{\mathrm{S}}^{n} \alpha^{2}\right)$ contributions to $p p \rightarrow W^{+}+n$ jets with stable on-shell $W$ bosons. At this order, reconciling the on-shell approximation with the presence of resonant $W$ propagators at amplitude level is nontrivial. However, the fact that resonant amplitudes contribute only through interference with non-resonant ones allowed us to regularise the poles of the relevant propagators with a technical width parameter, in a way that corresponds to a smooth and numerically negligible deformation with respect to the gauge-invariant on-shell limit. Using this approach we are going to implement $W$ boson decays in the narrow-width approximation in the near future.

We have presented various predictions for $W+$ multijet cross sections and distributions. For $p p \rightarrow W+1 j$, our NLO EW results confirm the well known Sudakov behaviour. The $W$-boson $p_{\mathrm{T}}$ distribution receives large negative EW corrections, which reach $-40 \%$ at $2 \mathrm{TeV}$ and are accompanied by NLO QCD corrections of similar size and opposite sign. Here, and in various other observables, the simultaneous presence of large EW and QCD corrections implies a sizable uncertainty related to the unknown $\mathrm{EW} \times \mathrm{QCD}$ corrections of NNLO type. For the distributions in the $p_{\mathrm{T}}$ of the first jet and in $H_{\mathrm{T}}^{\text {tot }}$ this problem becomes dramatic: in the TeV region NLO QCD corrections reach a factor ten, and the mere inclusion of NLO EW corrections at $\mathcal{O}\left(\alpha_{\mathrm{S}} \alpha^{2}\right)$ is completely insufficient. Actually, in the multi-TeV region we observe that NLO EW effects lead to a sizable positive correction, which arises from mixed EW-QCD real-emission contributions, while the expected Sudakov correction is completely suppressed.

As is well known, the explosion of NLO QCD corrections at high jet- $p_{\mathrm{T}}$ is due to the fact that $W+$ jets production with a very hard jet is dominated by $W+$ multijet configurations where the $W$ boson tends to be produced at moderate transverse momentum, while the transverse energy of the event is predominantly carried by two (or more) hard jets that recoil against each other. It is thus clear that, for a meaningful description of the hard-jet regime, NLO EW corrections must be extended to $W+n$-jet production with $n \geq 2$.

For $p p \rightarrow W^{+}+2$ jets, although $H_{\mathrm{T}}^{\text {tot }}$ remains quite sensitive to extra QCD radiation, the distributions in the $W$-boson and in the jet transverse momenta feature a good stability with respect to NLO QCD effects. Thus NLO QCD+EW predictions start providing a reliable theoretical description for these observables. At the TeV scale, the $p_{\mathrm{T}, W}$ distribution receives similar NLO EW corrections as in $W+1 j$ production, and also the jet- $p_{\mathrm{T}}$ distributions feature the expected Sudakov behaviour. The high relevance of the $H_{\mathrm{T}}^{\text {tot }}$ variable for new-physics searches and its strong sensitivity to QCD radiation motivate the extension of NLO QCD+EW calculations up to $p p \rightarrow W+3 j$, where $H_{\mathrm{T}}^{\text {tot }}$ starts to be stable with respect to NLO QCD corrections, thereby rendering NLO QCD+EW predictions more reliable. Similarly as for $W+2 j$, NLO EW corrections to $W+3 j$ are characterised by the expected Sudakov suppression in all $p_{\mathrm{T}}$ distributions. However the actual size of the corrections varies significantly, depending on the jet multiplicity of the considered process and on the individual $p_{\mathrm{T}}$-distribution. The magnitude of EW corrections at high energy can strongly depend on the type of observable as well. For instance, dijet invariant-mass distributions turn out to be completely insensitive to EW corrections, all the way up to the multi-TeV region. Finally, we pointed out that also photon-induced processes and subleading Born terms of $\mathcal{O}\left(\alpha^{n-1} \alpha^{2}\right)$ and $\mathcal{O}\left(\alpha^{n-2} \alpha^{3}\right)$, which result from EW contributions to the matrix elements, can have a sizable impact in the $\mathrm{TeV}$ region. 
In summary, EW correction effects in $W$ +multijet production feature a nontrivial dependence on the jet multiplicity, as well as on various kinematical parameters. Their sizable impact at high energies will play a key role for tests of the Standard Model and for many BSM searches based on signatures with jets, leptons and missing energy at the $\mathrm{TeV}$ scale. In a forthcoming publication we plan to present more detailed phenomenological results for vector-boson plus multi-jet production, including the case of $W^{-}$and $Z$ bosons as well as leptonic vector-boson decays. Our results motivate also further important developments of $\mathrm{NLO} \mathrm{QCD}+\mathrm{EW}$ simulations of vector-boson production in association with multiple jets, including matching to the parton shower and, ultimately, the extension of multi-jet merging techniques to NLO QCD+EW simulations.

\section{Acknowledgments}

We thank A. Denner, S. Dittmaier and L. Hofer for providing us with the one-loop tensorintegral library CoLLIER. We are grateful to F. Cascioli for collaboration at the initial stage of this project. This research was supported in part by the Swiss National Science Foundation (SNF) under contract PP00P2-128552 and by the Research Executive Agency (REA) of the European Union under the Grant Agreements PITN-GA-2010-264564 (LHCPhenoNet), PITN-GA-2012-315877 (MCnet) and PITN-GA-2012-316704 (HiggsTools). We thank the Munich Institute for Astro- and Particle Physics (MIAPP) of the DFG cluster of excellence "Origin and Structure of the Universe" for the hospitality during the completion of this work.

Open Access. This article is distributed under the terms of the Creative Commons Attribution License (CC-BY 4.0), which permits any use, distribution and reproduction in any medium, provided the original author(s) and source are credited.

\section{References}

[1] ATLAS collaboration, Measurement of the production cross section for $W^{-}$bosons in association with jets in pp collisions at $\sqrt{s}=7 \mathrm{TeV}$ with the ATLAS detector, Phys. Lett. B 698 (2011) 325 [arXiv: 1012.5382] [INSPIRE].

[2] ATLAS collaboration, Study of jets produced in association with a $W$ boson in pp collisions at $\sqrt{s}=7 \mathrm{TeV}$ with the ATLAS detector, Phys. Rev. D 85 (2012) 092002 [arXiv:1201.1276] [INSPIRE].

[3] ATLAS collaboration, Measurements of the $W$ production cross sections in association with jets with the ATLAS detector, Eur. Phys. J. C 75 (2015) 82 [arXiv:1409.8639] [InSPIRE].

[4] CMS collaboration, Jet production rates in association with $W$ and $Z$ bosons in pp collisions at $\sqrt{s}=7 \mathrm{TeV}$, JHEP 01 (2012) 010 [arXiv:1110.3226] [INSPIRE].

[5] CMS collaboration, Study of the dijet mass spectrum in $p p \rightarrow W+$ jets events at $\sqrt{s}=7$ TeV, Phys. Rev. Lett. 109 (2012) 251801 [arXiv:1208.3477] [INSPIRE].

[6] CMS collaboration, Differential cross section measurements for the production of a $W$ boson in association with jets in proton-proton collisions at $\sqrt{s}=7 \mathrm{TeV}$, Phys. Lett. B 741 (2015) 12 [arXiv:1406 .7533] [inSPIRE]. 
[7] P.B. Arnold and M.H. Reno, The complete computation of high $p_{T} W$ and $Z$ production in $2^{\text {nd }}$ order QCD, Nucl. Phys. B 319 (1989) 37 [Erratum ibid. B 330 (1990) 284] [InSPIRE].

[8] P.B. Arnold, R.K. Ellis and M.H. Reno, High $p_{T} W$ and $Z$ production at the Tevatron, Phys. Rev. D 40 (1989) 912 [inSPIRE].

[9] R.K. Ellis and S. Veseli, Strong radiative corrections to $W b \bar{b}$ production in $p \bar{p}$ collisions, Phys. Rev. D 60 (1999) 011501 [hep-ph/9810489] [INSPIRE].

[10] J.M. Campbell and R.K. Ellis, Next-to-leading order corrections to $W+2$ jet and $Z+2$ jet production at hadron colliders, Phys. Rev. D 65 (2002) 113007 [hep-ph/0202176] [INSPIRE].

[11] F. Febres Cordero, L. Reina and D. Wackeroth, NLO QCD corrections to $W$ boson

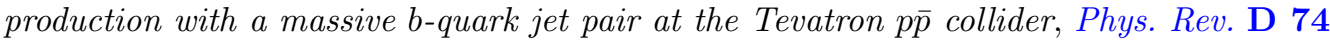
(2006) 034007 [hep-ph/0606102] [INSPIRE].

[12] J.M. Campbell, R.K. Ellis, F. Maltoni and S. Willenbrock, Production of a $W$ boson and two jets with one $b^{-}$quark tag, Phys. Rev. D 75 (2007) 054015 [hep-ph/0611348] [INSPIRE].

[13] J.M. Campbell et al., Associated production of a $W$ boson and one $b$ jet, Phys. Rev. D 79 (2009) 034023 [arXiv:0809.3003] [INSPIRE].

[14] F. Febres Cordero, L. Reina and D. Wackeroth, $W$ - and $Z$-boson production with a massive bottom-quark pair at the Large Hadron Collider, Phys. Rev. D 80 (2009) 034015 [arXiv:0906.1923] [INSPIRE].

[15] S. Badger, J.M. Campbell and R.K. Ellis, QCD corrections to the hadronic production of a heavy quark pair and a $W$-boson including decay correlations, JHEP 03 (2011) 027 [arXiv: 1011.6647] [INSPIRE].

[16] R. Frederix et al., $W$ and $Z / \gamma *$ boson production in association with a bottom-antibottom pair, JHEP 09 (2011) 061 [arXiv:1106.6019] [INSPIRE].

[17] J.M. Campbell, F. Caola, F. Febres Cordero, L. Reina and D. Wackeroth, NLO QCD predictions for $W+1$ jet and $W+2$ jet production with at least one $b$ jet at the $7 \mathrm{TeV} L \mathrm{LCC}$, Phys. Rev. D 86 (2012) 034021 [arXiv:1107.3714] [INSPIRE].

[18] R.K. Ellis, Z. Kunszt, K. Melnikov and G. Zanderighi, One-loop calculations in quantum field theory: from Feynman diagrams to unitarity cuts, Phys. Rept. 518 (2012) 141 [arXiv:1105.4319] [INSPIRE].

[19] H. Ita, SUSY theories and QCD: numerical approaches, J. Phys. A 44 (2011) 454005 [arXiv:1109.6527] [INSPIRE].

[20] C.F. Berger et al., Precise predictions for $W+3$ jet production at hadron colliders, Phys. Rev. Lett. 102 (2009) 222001 [arXiv:0902.2760] [INSPIRE].

[21] R.K. Ellis, K. Melnikov and G. Zanderighi, Generalized unitarity at work: first NLO QCD results for hadronic $W+3$ jet production, JHEP 04 (2009) 077 [arXiv:0901.4101] [INSPIRE].

[22] R.K. Ellis, K. Melnikov and G. Zanderighi, $W+3$ jet production at the Tevatron, Phys. Rev. D 80 (2009) 094002 [arXiv: 0906.1445] [INSPIRE].

[23] C.F. Berger et al., Next-to-leading order QCD predictions for $W+3$-jet distributions at hadron colliders, Phys. Rev. D 80 (2009) 074036 [arXiv:0907.1984] [INSPIRE]. 
[24] C.F. Berger et al., Precise predictions for $W+4$ jet production at the Large Hadron Collider, Phys. Rev. Lett. 106 (2011) 092001 [arXiv: 1009.2338] [INSPIRE].

[25] Z. Bern et al., Next-to-leading order W+5-jet production at the LHC, Phys. Rev. D 88 (2013) 014025 [arXiv:1304.1253] [INSPIRE].

[26] V.S. Fadin, L.N. Lipatov, A.D. Martin and M. Melles, Resummation of double logarithms in electroweak high-energy processes, Phys. Rev. D 61 (2000) 094002 [hep-ph/9910338] [INSPIRE].

[27] J.H. Kühn, A.A. Penin and V.A. Smirnov, Summing up subleading Sudakov logarithms, Eur. Phys. J. C 17 (2000) 97 [hep-ph/9912503] [INSPIRE].

[28] A. Denner and S. Pozzorini, One loop leading logarithms in electroweak radiative corrections. 1. Results, Eur. Phys. J. C 18 (2001) 461 [hep-ph/0010201] [INSPIRE].

[29] A. Denner and S. Pozzorini, One loop leading logarithms in electroweak radiative corrections. 2. Factorization of collinear singularities, Eur. Phys. J. C 21 (2001) 63 [hep-ph/0104127] [INSPIRE].

[30] M. Ciafaloni, P. Ciafaloni and D. Comelli, Bloch-Nordsieck violating electroweak corrections to inclusive TeV scale hard processes, Phys. Rev. Lett. 84 (2000) 4810 [hep-ph/0001142] [INSPIRE].

[31] U. Baur, Weak boson emission in hadron collider processes, Phys. Rev. D 75 (2007) 013005 [hep-ph/0611241] [INSPIRE].

[32] K. Mishra et al., Electroweak corrections at high energies, arXiv:1308.1430 [INSPIRE].

[33] J.H. Kühn, A. Kulesza, S. Pozzorini and M. Schulze, Electroweak corrections to large transverse momentum production of $W$ bosons at the LHC, Phys. Lett. B 651 (2007) 160 [hep-ph/0703283] [INSPIRE].

[34] J.H. Kühn, A. Kulesza, S. Pozzorini and M. Schulze, Electroweak corrections to hadronic production of $W$ bosons at large transverse momenta, Nucl. Phys. B 797 (2008) 27 [arXiv: 0708.0476] [INSPIRE].

[35] A. Denner, S. Dittmaier, T. Kasprzik and A. Mück, Electroweak corrections to $W+$ jet hadroproduction including leptonic $W$-boson decays, JHEP 08 (2009) 075 [arXiv:0906.1656] [INSPIRE].

[36] M. Chiesa et al., Electroweak Sudakov corrections to new physics searches at the LHC, Phys. Rev. Lett. 111 (2013) 121801 [arXiv: 1305.6837] [INSPIRE].

[37] S. Actis, A. Denner, L. Hofer, A. Scharf and S. Uccirati, Recursive generation of one-loop amplitudes in the standard model, JHEP 04 (2013) 037 [arXiv:1211.6316] [INSPIRE].

[38] S. Actis, A. Denner, L. Hofer, A. Scharf and S. Uccirati, EW and QCD one-loop amplitudes with RECOLA, PoS (RADCOR 2013) 034 [arXiv: 1311.6662] [INSPIRE].

[39] A. Denner, L. Hofer, A. Scharf and S. Uccirati, Electroweak corrections to $Z+2$ jets production at the $L H C$, POS (RADCOR 2013) 019 [arXiv:1311.5336] [INSPIRE].

[40] A. Denner, L. Hofer, A. Scharf and S. Uccirati, Electroweak corrections to lepton pair production in association with two hard jets at the LHC, JHEP 01 (2015) 094 [arXiv: 1411.0916] [INSPIRE].

[41] S. Frixione, V. Hirschi, D. Pagani, H.S. Shao and M. Zaro, Weak corrections to Higgs hadroproduction in association with a top-quark pair, JHEP 09 (2014) 065 [arXiv: 1407.0823] [INSPIRE]. 
[42] J. Alwall et al., The automated computation of tree-level and next-to-leading order differential cross sections and their matching to parton shower simulations, JHEP 07 (2014) 079 [arXiv: 1405.0301] [inSPIRE].

[43] G. Cullen et al., GoSAm-2.0: a tool for automated one-loop calculations within the standard model and beyond, Eur. Phys. J. C 74 (2014) 3001 [arXiv: 1404.7096] [INSPIRE].

[44] F. Cascioli, P. Maierhöfer and S. Pozzorini, Scattering amplitudes with open loops, Phys. Rev. Lett. 108 (2012) 111601 [arXiv:1111.5206] [INSPIRE].

[45] S. Höche and M. Schönherr, in preparation.

[46] T. Gleisberg and F. Krauss, Automating dipole subtraction for QCD NLO calculations, Eur. Phys. J. C 53 (2008) 501 [arXiv:0709.2881] [InSPIRE].

[47] T. Gleisberg et al., Event generation with SHERPA 1.1, JHEP 02 (2009) 007 [arXiv: 0811.4622] [INSPIRE].

[48] F. Cascioli et al., Precise Higgs-background predictions: merging NLO QCD and squared quark-loop corrections to four-lepton $+0,1$ jet production, JHEP 01 (2014) 046 [arXiv: 1309.0500] [INSPIRE].

[49] F. Cascioli, P. Maierhöfer, N. Moretti, S. Pozzorini and F. Siegert, NLO matching for $t \bar{t} b \bar{b}$ production with massive b-quarks, Phys. Lett. B 734 (2014) 210 [arXiv:1309.5912] [INSPIRE].

[50] F. Cascioli, S. Kallweit, P. Maierhöfer and S. Pozzorini, A unified NLO description of top-pair and associated Wt production, Eur. Phys. J. C 74 (2014) 2783 [arXiv:1312.0546] [INSPIRE].

[51] P. Maierhöfer and A. Papaefstathiou, Higgs boson pair production merged to one jet, JHEP 03 (2014) 126 [arXiv: 1401.0007] [INSPIRE].

[52] S. Höche et al., Next-to-leading order QCD predictions for top-quark pair production with up to two jets merged with a parton shower, arXiv:1402.6293 [INSPIRE].

[53] S. Höche et al., Triple vector boson production through Higgs-Strahlung with NLO multijet merging, Phys. Rev. D 89 (2014) 093015 [arXiv: 1403.7516] [INSPIRE].

[54] G. Abelof, A. Gehrmann-De Ridder, P. Maierhöfer and S. Pozzorini, NNLO QCD

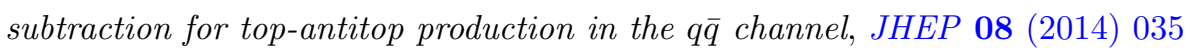
[arXiv: 1404.6493] [INSPIRE].

[55] M. Grazzini, S. Kallweit, D. Rathlev and A. Torre, $Z \gamma$ production at hadron colliders in NNLO QCD, Phys. Lett. B 731 (2014) 204 [arXiv:1309.7000] [INSPIRE].

[56] F. Cascioli et al., ZZ production at hadron colliders in NNLO QCD, Phys. Lett. B 735 (2014) 311 [arXiv: 1405.2219] [INSPIRE].

[57] T. Gehrmann et al., $W^{+} W^{-}$production at hadron colliders in next to next to leading order QCD, Phys. Rev. Lett. 113 (2014) 212001 [arXiv:1408.5243] [INSPIRE].

[58] A. Denner, S. Dittmaier, S. Kallweit and S. Pozzorini, NLO QCD corrections to WWbb production at hadron colliders, Phys. Rev. Lett. 106 (2011) 052001 [arXiv:1012.3975] [INSPIRE].

[59] A. Denner, S. Dittmaier, S. Kallweit and S. Pozzorini, NLO QCD corrections to off-shell top-antitop production with leptonic decays at hadron colliders, JHEP 10 (2012) 110 [arXiv:1207.5018] [INSPIRE]. 
[60] A. Denner, L. Hosekova and S. Kallweit, $N L O$ QCD corrections to $W^{+} W^{+} j j$ production in vector-boson fusion at the LHC, Phys. Rev. D 86 (2012) 114014 [arXiv:1209.2389] [INSPIRE].

[61] S. Catani and M. Grazzini, An NNLO subtraction formalism in hadron collisions and its application to Higgs boson production at the LHC, Phys. Rev. Lett. 98 (2007) 222002 [hep-ph/0703012] [INSPIRE].

[62] S. Höche, F. Krauss, M. Schönherr and F. Siegert, $W+n$-jet predictions at the Large Hadron Collider at next-to-leading order matched with a parton shower, Phys. Rev. Lett. 110 (2013) 052001 [arXiv: 1201.5882] [INSPIRE].

[63] S. Höche, F. Krauss, M. Schönherr and F. Siegert, QCD matrix elements + parton showers: the NLO case, JHEP 04 (2013) 027 [arXiv:1207.5030] [inSPIRE].

[64] S. Catani and M.H. Seymour, A general algorithm for calculating jet cross-sections in NLO QCD, Nucl. Phys. B 485 (1997) 291 [Erratum ibid. B 510 (1998) 503] [hep-ph/9605323] [INSPIRE].

[65] S. Catani, S. Dittmaier, M.H. Seymour and Z. Trócsányi, The dipole formalism for next-to-leading order QCD calculations with massive partons, Nucl. Phys. B 627 (2002) 189 [hep-ph/0201036] [INSPIRE].

[66] J. Butterworth et al., Les Houches 2013: physics at TeV colliders: standard model working group report, arXiv:1405.1067 [INSPIRE].

[67] A.D. Martin, R.G. Roberts, W.J. Stirling and R.S. Thorne, Parton distributions incorporating QED contributions, Eur. Phys. J. C 39 (2005) 155 [hep-ph/0411040] [INSPIRE].

[68] NNPDF collaboration, R.D. Ball et al., Parton distributions with QED corrections, Nucl. Phys. B 877 (2013) 290 [arXiv: 1308.0598] [INSPIRE].

[69] E.W.N. Glover and A.G. Morgan, Measuring the photon fragmentation function at LEP, Z. Phys. C 62 (1994) 311 [INSPIRE].

[70] A. Gehrmann-De Ridder, T. Gehrmann and E.W.N. Glover, Radiative corrections to the photon +1 jet rate at LEP, Phys. Lett. B 414 (1997) 354 [hep-ph/9705305] [inSPIRE].

[71] A. Gehrmann-De Ridder and E.W.N. Glover, Final state photon production at LEP, Eur. Phys. J. C 7 (1999) 29 [hep-ph/9806316] [InSPIRE].

[72] ALEPH collaboration, D. Buskulic et al., First measurement of the quark to photon fragmentation function, Z. Phys. C 69 (1996) 365 [InSPIRE].

[73] L. Bourhis, M. Fontannaz and J.P. Guillet, Quarks and gluon fragmentation functions into photons, Eur. Phys. J. C 2 (1998) 529 [hep-ph/9704447] [INSPIRE].

[74] M. Fontannaz, J.P. Guillet and G. Heinrich, Isolated prompt photon photoproduction at NLO, Eur. Phys. J. C 21 (2001) 303 [hep-ph/0105121] [InSPIRE].

[75] M. Klasen, Theory of hard photoproduction, Rev. Mod. Phys. 74 (2002) 1221 [hep-ph/0206169] [INSPIRE].

[76] A. Gehrmann-De Ridder, T. Gehrmann and E. Poulsen, Measuring the photon fragmentation function at HERA, Eur. Phys. J. C 47 (2006) 395 [hep-ph/0604030] [INSPIRE].

[77] F. Krauss, R. Kuhn and G. Soff, $A M E G I C++1.0$ : a matrix element generator in $C++$, JHEP 02 (2002) 044 [hep-ph/0109036] [INSPIRE]. 
[78] T. Gleisberg and S. Höche, Comix, a new matrix element generator, JHEP 12 (2008) 039 [arXiv:0808.3674] [INSPIRE].

[79] S. Alioli et al., Update of the Binoth Les Houches accord for a standard interface between Monte Carlo tools and one-loop programs, Comput. Phys. Commun. 185 (2014) 560 [arXiv:1308.3462] [INSPIRE].

[80] J. Bellm et al., HERWIG++ 2.7 release note, arXiv:1310.6877 [INSPIRE].

[81] A. van Hameren, Multi-gluon one-loop amplitudes using tensor integrals, JHEP 07 (2009) 088 [arXiv: 0905.1005] [INSPIRE].

[82] A. Denner, S. Dittmaier and L. Hofer, COLLIER - a fortran-library for one-loop integrals, PoS (LL2014) 071 [arXiv: 1407.0087] [INSPIRE].

[83] A. Denner and S. Dittmaier, Reduction of one loop tensor five point integrals, Nucl. Phys. B 658 (2003) 175 [hep-ph/0212259] [INSPIRE].

[84] A. Denner and S. Dittmaier, Reduction schemes for one-loop tensor integrals, Nucl. Phys. B 734 (2006) 62 [hep-ph/0509141] [INSPIRE].

[85] A. Denner and S. Dittmaier, Scalar one-loop 4-point integrals, Nucl. Phys. B 844 (2011) 199 [arXiv: 1005.2076] [INSPIRE].

[86] G. Ossola, C.G. Papadopoulos and R. Pittau, Reducing full one-loop amplitudes to scalar integrals at the integrand level, Nucl. Phys. B 763 (2007) 147 [hep-ph/0609007] [INSPIRE].

[87] G. Ossola, C.G. Papadopoulos and R. Pittau, CutTools: a program implementing the OPP reduction method to compute one-loop amplitudes, JHEP 03 (2008) 042 [arXiv:0711.3596] [INSPIRE].

[88] P. Mastrolia, G. Ossola, T. Reiter and F. Tramontano, Scattering amplitudes from unitarity-based reduction algorithm at the integrand-level, JHEP 08 (2010) 080 [arXiv:1006.0710] [INSPIRE].

[89] A. van Hameren, OneLOop: for the evaluation of one-loop scalar functions, Comput. Phys. Commun. 182 (2011) 2427 [arXiv:1007.4716] [INSPIRE].

[90] G. Ossola, C.G. Papadopoulos and R. Pittau, On the rational terms of the one-loop amplitudes, JHEP 05 (2008) 004 [arXiv:0802.1876] [inSPIRE].

[91] T. Binoth, J.P. Guillet and G. Heinrich, Algebraic evaluation of rational polynomials in one-loop amplitudes, JHEP 02 (2007) 013 [hep-ph/0609054] [INSPIRE].

[92] A. Bredenstein, A. Denner, S. Dittmaier and S. Pozzorini, NLO QCD corrections to t $\bar{t} b \bar{b}$ production at the LHC: 1. Quark-antiquark annihilation, JHEP 08 (2008) 108 [arXiv: 0807.1248] [INSPIRE].

[93] P. Draggiotis, M.V. Garzelli, C.G. Papadopoulos and R. Pittau, Feynman rules for the rational part of the QCD 1-loop amplitudes, JHEP 04 (2009) 072 [arXiv:0903.0356] [INSPIRE].

[94] M.V. Garzelli, I. Malamos and R. Pittau, Feynman rules for the rational part of the electroweak 1-loop amplitudes, JHEP 01 (2010) 040 [Erratum ibid. 10 (2010) 097] [arXiv:0910.3130] [INSPIRE].

[95] M.V. Garzelli, I. Malamos and R. Pittau, Feynman rules for the rational part of the electroweak 1-loop amplitudes in the $R_{\xi}$ gauge and in the Unitary gauge, JHEP 01 (2011) 029 [arXiv: 1009.4302] [INSPIRE]. 
[96] M.V. Garzelli and I. Malamos, R2SM: a package for the analytic computation of the $R_{2}$ rational terms in the standard model of the electroweak interactions, Eur. Phys. J. C 71 (2011) 1605 [arXiv: 1010.1248] [INSPIRE].

[97] H.-S. Shao, Y.-J. Zhang and K.-T. Chao, Feynman rules for the rational part of the standard model one-loop amplitudes in the 't Hooft-Veltman $\gamma_{5}$ scheme, JHEP 09 (2011) 048 [arXiv: 1106.5030] [INSPIRE].

[98] A. Denner, Techniques for calculation of electroweak radiative corrections at the one loop level and results for $W$ physics at LEP-200, Fortsch. Phys. 41 (1993) 307 [arXiv: 0709.1075] [INSPIRE].

[99] A. Denner, S. Dittmaier, M. Roth and L.H. Wieders, Electroweak corrections to charged-current $e^{+} e^{-} \rightarrow 4$ fermion processes: technical details and further results, Nucl. Phys. B 724 (2005) 247 [Erratum ibid. B 854 (2012) 504] [hep-ph/0505042] [INSPIRE].

[100] S. Dittmaier, A general approach to photon radiation off fermions, Nucl. Phys. B 565 (2000) 69 [hep-ph/9904440] [INSPIRE].

[101] S. Dittmaier, A. Kabelschacht and T. Kasprzik, Polarized QED splittings of massive fermions and dipole subtraction for non-collinear-safe observables, Nucl. Phys. B 800 (2008) 146 [arXiv: 0802.1405] [INSPIRE].

[102] T. Gehrmann and N. Greiner, Photon radiation with MadDipole, JHEP 12 (2010) 050 [arXiv: 1011.0321] [INSPIRE].

[103] A. Bredenstein, A. Denner, S. Dittmaier and S. Pozzorini, NLO QCD corrections to top anti-top bottom anti-bottom production at the LHC: 2. Full hadronic results, JHEP 03 (2010) 021 [arXiv: 1001.4006] [INSPIRE].

[104] Z. Nagy and Z. Trócsányi, Next-to-leading order calculation of four jet observables in electron positron annihilation, Phys. Rev. D 59 (1999) 014020 [Erratum ibid. D 62 (2000) 099902] [hep-ph/9806317] [INSPIRE].

[105] Z. Nagy, Next-to-leading order calculation of three jet observables in hadron hadron collision, Phys. Rev. D 68 (2003) 094002 [hep-ph/0307268] [InSPIRE].

[106] J.M. Campbell, R.K. Ellis and F. Tramontano, Single top production and decay at next-to-leading order, Phys. Rev. D 70 (2004) 094012 [hep-ph/0408158] [INSPIRE].

[107] G. Bevilacqua, M. Czakon, C.G. Papadopoulos, R. Pittau and M. Worek, Assault on the $N L O$ wishlist: $p p \rightarrow t \bar{t} b \bar{b}, J H E P 09$ (2009) 109 [arXiv:0907.4723] [INSPIRE].

[108] J.M. Campbell and F. Tramontano, Next-to-leading order corrections to Wt production and decay, Nucl. Phys. B 726 (2005) 109 [hep-ph/0506289] [INSPIRE].

[109] R. Frederix, T. Gehrmann and N. Greiner, Integrated dipoles with MadDipole in the MadGraph framework, JHEP 06 (2010) 086 [arXiv: 1004.2905] [INSPIRE].

[110] M. Cacciari, G.P. Salam and G. Soyez, The anti-kt jet clustering algorithm, JHEP 04 (2008) 063 [arXiv:0802.1189] [INSPIRE]. 